\title{
NONLINEAR MECHANISMS FOR DRJFT WAVE SATURATION AND INDUCED PARTICLE TRANSPORT
}

\author{
A. M. Dimits \\ Laboratory for Plasma Researcl \\ University of Maryland, College Park, Maryland 20742 \\ and \\ PPPL--2659 \\ W. W. Lee \\ Princeton Plasma Physics Laboratory \\ DE90 00448: \\ Princeton University \\ Princet.Jn, New Jersey 08543
}

A detailed theoretical study of the nonlinear dynamics of gyrokinetic particle simulations of electrostatic collisionless and weakly collisionai drift waves is presented. In previous studies it was shown that, in the nonlinearly saturated phase of the evolution, the saturation levels and especially the particle fluxes have an unexpected dependence on collisionality. In this paper, the explanations for these collisionality dependences are found to be as follows: The saturation level is determined by a balance between the electron and ion fluxes. The ion flux is small for levels of the potential below an $\boldsymbol{E} \times \boldsymbol{B}$-trapping threshold and increases sharply once this threshold is crossed. Due to the presence of resonant electrons, the electron flux has a much smoother dependence on the potential. In the $2 \frac{1}{2}$-dimensional ("pseudo-3D") geometry, the electrons are accelerated away from the resonance as they diffuse spatially, resulting in an inhibition of their diffusion. Collisions and three-dimensional effects can repopulate the resonance thereby increasing the value of the particle flux.

\section{DISCLAIMER}

This report was prepared as an aceoum of work sponsored by an agency of the United States Government. Neither the United States Government nor any agency thercof, nor any of their err.ployees, makes any warranty, express or implied, or assumes any legal liability or responsibility for the accuracy. completeness, or usefulness of any information, apparatus, pfoduct, or process disclosed, or represents that its ust would not infringe privately owned rights. Refer. ence herein to any specific commercial product, process, or service by trade name, isademark. manufacturer. of otherwise does not necessarily constitute or imply its endorsement, recommendation, or favoring by the United States Governmen: or any agency thereof. The views and opinions of auttors expressed herein do not necessarily state or reflecl those of the United States Government of any agency thereor. 


\section{INTRODUCTION}

In this paper, we report a theoretical investigation of the nonlinear behavior of "2 $\frac{1}{2}$-dimensional" gyrolinetic particle simulations of collisionless and weaklycollisional drift waves in a periodic shear-free slab geometry. The gyrokinetic particle simulation method takes low frequency $\left(\omega \ll \Omega_{i}\right)$ nonlinear kinetic effects fully into account.

The term $2 \frac{1}{2}$-dimensional may be defined with respect to the fully threedimensional geometry by taking all functions of position to be independent of one of the spatial coordinates. If the magnetic field is not quite aligned with this ignorable coordinate, then this geometry allows for effects due to the parallel streaming of particles along the magnetic field lines, such as the near-thermal-equilibrium electron response to the wave fields (the "adiabatic response"). This geometry has also been termed "pseudo-three-dimensional."

The instabilities considered here are unstable drift waves. They arise because of the balance between the near-adiabatic electron response $\delta n / n \simeq e \phi / T_{e}$ and the ion density perturbations due to the $\boldsymbol{E} \times \boldsymbol{B}$ advection of ions from an jon density distribution with a nonzero density gradient, and are driven unstable by the combination of inverse electron Landau damping and finite ion-gyroradius or polarization-drift effects. The main results of the linear theory have been summarized by Federici, Lee, and Tang, ${ }^{2}$ and will be repeated in Sec. III.

The simulation runs studied here include those reported in published works of W. W. Lee and co-workers, ${ }^{3,2}$ as well as other runs carried out by the authors since those papers were written. They show the expected linear growth phase, a saturation phase, and a "steady-state phase." The behavior observed in the steady-state phase has a coherent aspect, being dominated by a few of the lowest available modes, and an incoherent aspect, in the random time dependence of the amplitudes of these modes. Both the saturation levels and the particle flux increase with electron collisionality. ${ }^{2}$ This was unexpected since the collisions are too weak to affect the linear growth rates significantly. The difficulty in understanding this dependence of the saturation levels and especially the particle fluxes on the collisionality motivated the present work.

Drift instabilities are wiclely regarded as important candidates for the anomalous thermal transport in magnetic plasma confinement devices, both in the bulk plasma and at the edge." There are drift-type modes unstable in most of the parameter space spanned by such plasmas. It is, therefore, important to understand how to suppress them or, failing that, to be able to estimate the anomalous transport that they produce. In order to do the latter properly, it is necessary to understand the nonlinear evolution resulting from these instabilities. The development of credible predictive first principles theories has proven to be a difficult task, and a posteri- 
ori confirmation is generally necessary. Computer simulations are a valuable tool both for testing existing nonlinear theories and, where these are found to fail, for stimulating and guiding the development of new ones. The gyrokinetic particle simulation method shows promise for the treatment of microinstabilities-i.e., instabilities which involve velocity-space effects (as well as macroinstabilities-i.e., fluid instabilities), in quite realistic situations with the current generation of supercomputers. ${ }^{5}$ For phenomena occurring on time scales much longer than the ion gyroperiod, this method is superior to simulations of the exact particle dynamics, since it can treat finite gyroradius effects without having to resolve the details of the gyromotion. ${ }^{5}$

Electrostatic collisionless drift waves in a shear-free slab, although too simplistic to provide a credible explanation for anomalous transport in magnetic confinement devices, are of interest for the following reasons. They represent a simple test case, of gradient-driven microinstabilities, for the gyrokinetic particle simulation method. These simulations have revealed unexpected coherent nonlinear phenomena and unexpected parameter dependences of the particle flux (in the direction opposite to that of the mean density gradient) and the saturation levels. ${ }^{3,2}$ Furthermore, similar phenomena are seen in simulations of the $\eta_{\mathrm{i}}$ mode, ${ }^{7}$ which has been invoked to explain ion thermal transport in tokamaks. ${ }^{8}$ Thus, the understanding of these simulations may provide important insight into the behavior of more realistic cases.

In this paper we provide an understanding of the dynamics of the saturated states of the above-mentioned simulations. Our strategy is as follows: First, the evolution of test-gyrocenter densities and fluxes resulting from electrostatic potentials of the form of those seen in the gyrokinetic simulations was studied. These studies, in addition to determining the parameter dependences of the gyrocenter fluxes, elucidate the physical mechanisms by which the electric field of the waves causes the fluxes. The electron and ion fluxes have different parametric dependences on the mean saturation level of the electrostatic potential (as well as on other parameters) and have a well-defined intersection. This intersection determines the self-consistent saturation level, as in the resonance-broadening model of Dupree. ${ }^{9}$ This approach can also be regarded as a nonlinear generalization of the standard procedure of linear theory in which the linear particle responses are computed and then inserted into the field equations to obtain the dispersion relation. We have obtained successful qualitative and, in many cases, quantitative explanations of most of the main observed properties of the saturated states of these gyrohinetic simulation runs. The understanding of this system also forms a basis for future attempts to understand the full three-dimensional simulations.

We now review and discuss the relevance of the existing nonlinear models of drift waves to the gyrokinetic simulation results. Most of the theoretical approaches have tended to concentrate on a single nonlinear mechanism. One class of approaches 
treats the electrons linearly and the ions nonlinearly. Examples are the work of Dupree, ${ }^{9}$ Terry and Horton, ${ }^{10,11}$ and Waltz. ${ }^{12}$ In Dupree's resonance-broadening theory, applied to the particular case of the current-driven drift wave, the instability grows until the ion diffusion shuts off the growth. In this sense, Dupree's theory is similar to the situation in the simulations studied here except that the latter are far more coherent. In the case of drift waves, the nonlinearity in the ion dynamics is necessitated by a steady-state particle flux since a steady-state particle flux is inconsistent with the cold (of relevance here) linear ion dynamics. We will elaborate on this in Sec. V. Terry and Horton ${ }^{10,21}$ and Waltz ${ }^{12}$ have numerically and analytically studied similar model fluid systems in which the ions are nonlinear and the electron behavior is given by a fixed, model linear response. In their studies, the turbulent coupling, due to the $\mathbf{E} \times \boldsymbol{B}$ and polarization-drift nonlinearities, to linearly stable modes is found to be a more important influence on the saturation levels than was assumed in the model of Dupree. In our simulations, the strong dominance of certain modes is the main incoherent effect of the turbulent mode coupling. The remaining effects, for example anomalous diffusion, can be taken into account as coherent effects on these modes. The electron flux, however, is due to nonlinear $E \times B$ diffusion of resonant electrons and not to linear effects as in these references. Oraevskii, Sagdeev, Galeev, and Rudakov ${ }^{13}$ have considered nonlinear stability and saturation due to parallel trapping. Mode-coupling studies by Lee et $a l^{3}$ and Federici et $a l^{2}$ have shown, however, that the $E \times B$ nonlinearity is a crucial ingredient in the dynamics of the simulations. The papers of Ching, ${ }^{14}$ Hirshman, ${ }^{15}$ Kleva and Drake, ${ }^{16}$ and Horton ${ }^{17}$ deal with $E \times B$ drifts in wave fields obtained by superposing two waves of equal amplitude, one with wave number $\left(k_{x}, k_{y}\right)$ and the other with wave number $\left(k_{x},-k_{y}\right)$. They have, therefore, served as useful starting points for some of the work in this paper. Here, $x$ and $y$ are, for the moment, coordinates in the plane perpendicular to the uniform magnetic field such that the direction of propagation of the wave is in the $y$ direction. The modes that dominate the simulation results used in this paper are of this form. Ching ${ }^{14}$ carefully analyzed the ion gyrocenter trajectories ar.d obtained their Hamiltonian. The Hamiltonians derived in Sec. IV are generalizations of that obtained by Ching. Kleva and Drake ${ }^{16}$ and Horton ${ }^{17}$ studjed the effect of a second such pair of waves, moving with respect to the first, of possibly different wave numbers and amplitudes. They obtained stochasticity criteria for the particle motion in these waves, and concluded that the particle motion is stochastic if there is $E \times B$ trapping due to each of the waves separately and if the $y$ component of the $E \times B$ velocity is greater than the difference in the phase velocities. The test-gyrocenter problems studied here, while bearing similarities to situations studied before by Ching, ${ }^{14}$ Hirshman,${ }^{15}$ Horton, ${ }^{17}$ and Kleva and Drake, ${ }^{18}$ also possess some important differences. In our case, there is a dynamically important time dependence of the electrostatic potential, which lies primarily in the time-dependent energy 
exchange between the two lowest available modes. This differs from the situation studied in the work of Horton ${ }^{17}$ and Kleva and Drake, ${ }^{16}$ where the time dependence was due to other modes. The nature of this time dependence has an important effect on the character of the time-averaged ion and electron responses resulting from the $E \times B$ trapping. The contribution of the parallel acceleration to the ion Landau damping, considered by Ching, ${ }^{14}$ (linear or nonlinear) is ineffective as a saturation mecharism in the simulations considered here because the ions are essentially cold in the parallel direction. Note also that the existence of the Hamiltonians of Sec. IV for the $2 \frac{1}{2}$-dimensional ion motion inplies that the $E \times B$ motion of the ions will actually shut off the ion Landau damping instead of enhancing it as claimed by Ching. ${ }^{14}$ Lee et al. ${ }^{3}$ and Smith et al. ${ }^{18}$ considered saturation by coherent $\boldsymbol{E} \times \boldsymbol{B}$ advection of the resonant electrons which completely switches off the linear electron phase shift. This mechanism is relevant for cases where the flux is much less than quasilinear and in the initial stages of the saturation in other cases. When significant particle fluxes are present, however, an electron phase shift remains and the ion nonlinearities become an essential part of the saturation mechanism. Finally, the density distributions that result from and are advected by the $E \times B$ drift to produce the $E \times B$ diffusion and the regions of enhanced density require modes of quite high wave number for their adequate representation. The mode-coupling systems studied by Lee et al. ${ }^{3}$ and Federici et al. ${ }^{2}$ do not resolve the gyrocenter densities accurately enough to contain the effects of the $E \times B$ motion that control the particle fluxes which, in turn, affect the nonlinear dynamics of the particle sirnulations.

Briefly, the main results of this paper are as follows. The ion parallel motion is relatively unimportant, so the ion gyrocenters are adequately described by a fluid continuity equation. In the gyrokinetic system, the physical ion density consists of the ion gyrocenter density plus the contribution of the polarization drift to the density. This "polarization density" is important, and is accounted for in the gyrokinetic Poisson equation. Regions of enhanced density of both particle species form and grow near the maxima of the potential because of the $E \times B$ advection of very well $E \times B$-trapped regions of electron and ion gyrocenters in the direction opposite to the density gradient. There is an "advective component" of the particle flux due to $E \times B$ advection of these regions. There is an asymmetry between the quality of the $E \times B$ trapping near the potential maxima and minima, in that there is much better trapping near the maxima. This asymmetry is due partly to the method used to model the background density gradient and partly to self-consistent effects. The analysis of test-electron orbits shows that the parallel acceleration can be treated linearly in some cases, a point we have verified by using a partially linearized version of the gyrokinetic code. ${ }^{19}$ The electron flux is dominated by the $\boldsymbol{E} \times \boldsymbol{B}$ diffusion of the parallel-resonant electrons. In the $2 \frac{1}{2}$-dimensional model, the paraliel acceleration depletes the resonant electron population. The particle flux 
and the saturation level depend on the electron collisionality because the collisions constitute the only mechanism present that can repopulate the resonance. The energy exchange between the lowest modes of the potential is primarily the result of the $\boldsymbol{E} \times \boldsymbol{B}$ advection of electrons and ions from nonuniform density distributions, and the electron $\boldsymbol{E} \times \boldsymbol{B}$ diffusion is a consequence of this energy exchange on the resonant electron population. The gyrokinetic Poisson equation forces the ions to have the same spatially averaged flux as the electrons (often termed "intrinsic ambipolarity"). The dependence of the nonlinear contribution to the ion flux on the mean saturation level is stronger than for the electron flux, so this ambipolarity requirement imposes a well-defined tnean saturation level. The saturated spectra are very strongly dominated by the lowest few modes of the potential even when these are not the most strongly unstable mod $\rho_{j .}$. This property of the spectrum may be due to a madified two-dimensional fluid inverse energy cascade. The value of the $2 \frac{1}{2}$-dimensional simulations is that they enable the careful study of the properties of a single resonance. This aids in the understanding of the properties of the three-dimensional simulations in which many such resonances may be present.

In more turbulent situations the coherent component of the flux will be less important. If the inhibition of the electron $\boldsymbol{E} \times \boldsymbol{B}$ diffusion by both coherent effects and resonance depopulation by the parallel acceleration is negligible, then the linear electron phase shift used by Dupree, ${ }^{9}$ Terry and Horton, ${ }^{10,11}$ and Waltz ${ }^{12}$ should quite accurately give the electron tlux. This will be shown in Sec. IX. In this case, the theory of Dupree (applied specifically to collisionless drift waves) would be applicable. Preliminary results from three-dimensional gyrokinetic simulations of drift waves ${ }^{20}$ indicate a particle flux significantly enhanced over the $2 \frac{1}{2}$-dimensional results, but still somewhat less than the expectation from the linear electron phase shift. The results of non-self-consistent analysis of the motion of guiding center electrons in three-dimensional wave fields suggest that the resonance depopulation should be much less important in three-dimensional simulations of drift waves. Thus, there is still an inhibition of the $\boldsymbol{E} \times \boldsymbol{B}$ diffusion due to coherence in the wave fields in these simulations. A careful study with a full three-dimensional gyrokinetic code still remains to be done.

The organization of this paper is as follows. In Sec. II, the nonlinear gyrokinetic simulation algorithm is described. We summarize the nonlinear electrostatic gyrokinetic equations of Lee ${ }^{6}$ and Dubin et al. ${ }^{21}$ the "multiscale model" used to model the effects of the background density gradient, the geometry, and some details of their implementation in particle simulations. In Sec. III, we discuss the main phenomena observed in the simulations and include a summary of the parameter values and the main results of the linear theory. Some of the phenomena have been reported before. ${ }^{2}$ In Sec. IV we derive the useful test-gyrocenter Hamiltonians for the cases of interest here as well as for nonzero shear. The non-self-consistent gyrocenter simulation codes, which prove extremely useful in bith guiding and confirming the 
theoretical understanding of the fully self-consistent gyrokinetic simulation results, are introduced in Sec. V. The relationship between the fluxes generated by those simulations to the behavior of the full code is also discussed. In Sec. VI we give the results of the non-self-consistent simulations for both the ions and electrons. In Sec. VII, we give a theoretical discussion of the ion and electron motion in terms of the instantaneous collisionless streamlines. Analytical estimates of the $E \times B$ diffusion due to the semi-coherent time-dependent wave fields seen in the full gyrokinetic simulations are given in Sec. VIII. In Sec. IX the understanding obtained in the earlier sections is used to interpret some aspects of the behavior of the gyrokinetic simulations and to relate these simulations to expectations from more complete models of nonlinear drift wave behavior. Finally, in Sec. X, we summarize the main results of this paper and give suggestions for further work.

\section{GYROKINETIC PARTICLE SIMULATION METHOD}

For the sake of completeness, we briefly review the gyrokinetic particle simulation method of W. W. Lee and co-workers, namely Lee, ${ }^{6}$ Dubin et al., ${ }^{21}$ Lee et al., ${ }^{3}$ Krommes et al., ${ }^{22}$ and Federici et al. ${ }^{2}$

The gyrokinetic system of equations of Lee ${ }^{6}$ and Dubin et al. ${ }^{21}$ is based on the well-known gyrokinetic ordering,

$$
\frac{e \Phi}{T} \sim \frac{\omega}{\Omega} \sim \frac{\rho}{L} \sim \epsilon \ll 1, \quad L \sim L_{\| l},
$$

where $q$ is the charge of the species under consideration, $\Phi$ is the electrostatic potential, $\mathrm{T}$ is the temperature, $\omega$ is the frequency of the perturbation, $\Omega$ is the cyclotron frequency, $\rho$ is the gyroradius, $L$ is a characteristic perpendicular equilibrium scale length of the system, and $L_{\|}$is the characteristic parallel wavelength of the perturbation. This system consists of the gyrokinetic Vlasov equation, for the gyrophase-averaged distribution function, and the gyrokinetic Poisson equation, which determines the electrostatic potential in terms of the gyrocenter densities of the electrons and ions.

The gyrokinetic Vlasov equation for a plasma in a uniform magnetic field is

$$
\frac{\partial F}{\partial t}+v_{\|} \hat{\boldsymbol{b}} \cdot \frac{\partial F}{\partial \boldsymbol{R}}-\frac{q}{m \Omega} \frac{\partial}{\partial \boldsymbol{R}} \cdot\left[\left(\frac{\partial \Psi}{\partial \boldsymbol{R}} \times \hat{\boldsymbol{b}}\right) F\right]-\frac{q}{m} \frac{\partial \Psi}{\partial \boldsymbol{R}} \cdot \hat{b} \frac{\partial F}{\partial v_{\|}}=C(F),
$$

where

$$
\begin{aligned}
\Psi(\boldsymbol{R}) & \doteq \bar{\phi}-\frac{q}{2 T}\left(\frac{v_{\mathrm{t}}}{\Omega}\right)^{2}\left|\frac{\partial \phi}{\partial R}\right|^{2} \\
\bar{\phi} & \doteq \sum_{\boldsymbol{k}} \phi(k) J_{0}\left(\frac{k_{\perp} v_{\perp}}{\Omega}\right) \exp (i k \cdot R)
\end{aligned}
$$




$$
\boldsymbol{R} \doteq \boldsymbol{x}+\frac{\boldsymbol{v}_{\perp} \times \hat{\boldsymbol{b}}}{\Omega},
$$

$\boldsymbol{x}$ is the particle position, $\boldsymbol{v}_{\perp}$ is the perpendicular velocity, $\vec{\phi}(\boldsymbol{R})$ is the gyrophaseaveraged electrostatic potential, $F\left(\boldsymbol{R}, \mu, v_{\|}, t\right)$ is the full gyrophase-averaged distribution function, $X$ is the $x$ component of $\boldsymbol{R}, \mu \doteq v_{\perp}^{2} / 2, \Omega \doteq \mathrm{g} B / \mathrm{mc}$ is the gyrofrequency, $v_{\mathrm{t}} \doteq \sqrt{T / m}$ is the thermal velocity, and $C(F)$ is the collision operator. The electrostatic potential $\phi$ is given by the gyrokinetic Poisson equation which, for a single ion species is

$$
\nabla^{2} \phi-\frac{\tau(\phi-\tilde{\phi})}{\lambda_{D}^{2}}+\left(\frac{\rho_{\mathrm{B}}}{\lambda_{D}}\right)^{2} \nabla_{\perp} \cdot\left[\frac{\left(n^{i}-n_{0}\right)}{n_{0}} \nabla_{\perp} \phi\right]=-4 \pi e\left(\bar{n}^{i}-n^{e}\right),
$$

where

$$
\begin{aligned}
\tilde{\phi}(\boldsymbol{x}) & \doteq \sum_{\boldsymbol{k}} \phi(\boldsymbol{k}) \Gamma_{0}\left(k_{\perp}^{2} \rho_{\mathrm{i}}^{2}\right) \exp (i \boldsymbol{k} \cdot \boldsymbol{x}) \\
\tilde{n}(\boldsymbol{x}) & \doteq \sum_{\boldsymbol{k}} \int F(\boldsymbol{k}) J_{0}\left(\frac{k_{\perp} v_{\perp}}{\Omega}\right) \exp (i \boldsymbol{k} \cdot \boldsymbol{R}) d \mu d v_{\|}
\end{aligned}
$$

and where $\tau \doteq T_{\mathrm{e}} / T_{\mathrm{i}}, \rho_{\mathrm{s}} \doteq \Omega_{\mathrm{i}}^{-1} \sqrt{T_{\mathrm{e}} / m_{\mathrm{i}}}$ is the ion-sound radius, $\rho_{\mathrm{i}} \doteq \Omega_{\mathrm{i}}^{-1} \sqrt{T_{\mathrm{i}} / m_{\mathrm{i}}}$ is the ion gyroradius, $k_{\perp}$ is the perpendicular wave number, $\lambda_{D} \doteq \sqrt{T_{e} / 4 \pi n_{0} e^{2}}$ is the electron Debye length, $n_{0}$ is the background ion number density, $T_{\alpha}, \pi^{\alpha}$, and $m_{\alpha}$ are, respectively, the temperature, the number density, and the particle mass of species $\alpha$, and $\Gamma_{0}(b) \doteq I_{0}(b) e^{-b}$. For ions, $C(F)=0$. For the electrons, finite gyroradius effects are negligible so that $\Psi=\bar{\phi}=\bar{\phi}=\phi$. The only effect of collisions retained is pitch-angle scattering for the electrons, which is taken into account via a Monte-Carlo model for the Lorentz collision operator, ${ }^{2}$

$$
C(F)=\frac{\nu_{\mathrm{ci}}}{2 \sin \theta} \frac{\partial}{\partial \theta}\left(\sin \theta \frac{\partial F_{\epsilon}}{\partial \theta}\right) .
$$

Here $\nu_{\mathrm{ei}}$ is the electron pitch-angle scattering frequency, which in these studies is taken to be velocity-independent, and $\theta$ (just for this equation) is the pitch angle. The intrinsic collision time due to the particle discreteness in the simulation is several orders of magnitude greater than the typical run time. ${ }^{2}$ The Lorentz operator is modelled using a Monte-Carlo model due to Shanny, Dawson, and Greene. ${ }^{23}$ For modelling purposes, the neglect of the velocity dependence of $\nu_{\mathrm{ei}}$ is satisfactory since the population of runaway electrons should be negligible. This is because the the parallel phase velocity of the waves is smaller than the electron thermal velocity. Simulations using (a Monte-Carlo model for) the Lenard-Bernstein model collision operator, ${ }^{24}$

$$
C(F)=\nu_{\mathrm{ei}} \frac{\partial}{\partial v_{\|}}\left[v_{\mathrm{te}}^{2} \frac{\partial f}{\partial v_{\|}}+v_{\|} f\right]
$$


which is a drag-diffusion model in $v_{\|}$only, yield similar results. This model is, therefore, useful in obtaining a qualitative understanding of the effect of the collisions in these simulations. The above equations are solved using somewhat standard particle simulation techniques. ${ }^{5}$

We now discuss some aspects of the method used to model the effects of the background density gradient. For initial studies, in which the main aim is to obtain insight into the physical mechanisms at work, a simulation representing a small portion of the entire discharge with a fixed background density gradient rather than the whole discharge has several advantages: Firstly, it enables concentration on relatively few physical mechanisms. Secondly, such a simulation can be run for much longer physical times. Thirdly, when periodic boundary conditions are imposed, it allows steady-state particle fluxes without particle sources or sinks. Simulations of drift instabilities with particle sources and sinks are subject to difficulties in interpretation. ${ }^{25}$

A method of modelling the effects of a fixed background density gradient, consistent with the above requirements, has been given by Lee, ${ }^{5}$ where it is termed the "multiple-scale model." The result is that the perpendicular configuration-space velocity in Eq. (1a) is modified by the addition of an extra term so that it becomes

$$
\boldsymbol{v}_{\text {perp }} \doteq \frac{d \boldsymbol{R}}{d t}=-\frac{q}{m \Omega}\left[\frac{\partial \Psi}{\partial \boldsymbol{R}}+\kappa \Psi\right] \times \hat{\boldsymbol{b}}
$$

where $\kappa \doteq-\nabla \ln n_{0}=\kappa \hat{x}$. This velocity has the important property

$$
\nabla \cdot v_{\text {perp }}=-\kappa \dot{X},
$$

where $\dot{X}=-(q / m \Omega)(\partial \Psi / \partial Y)$ is the $x$ component of $v_{\text {perp. }}$. The continuity equation for the configuration-space density can thus be expressed in the form

$$
\frac{d n}{d t}+\frac{\partial}{\partial z} J_{\| I}=\kappa n \dot{X}
$$

where $d / d t \doteq \partial / \partial t+v_{\text {perp }} \cdot \nabla$ is the convective time derivative and $J_{\|}$is the parallel current. Assume, for simplicity, that $J_{\|}=0$. Then, Eq. (6) states that as a group of particles is convected by $v_{\text {perp }}$ in the $x$ direction, its density increases due to the compression in the $y$ direction of the perpendicular configuration space element containing it. If the background density (of simulation particles) is flat, then this results in a density perturbation, as shown in Fig. 1 (a). Under the $J_{\|}=0$ assumption, density profiles proportional to $e^{x x}$ are unaffected by the flow given by Eq. (4) irrespective of $\Psi$, and are, therefore, equilibria of Eq. (6). The imposition of periodic boundary conditions, however, forces the mean large-scale simulation particle density to be flat. Thus, there is a permanently enforced separation between the mean particle density and the equilibrium pmiricle density. This is an 
alternative, and for the most part satisfactory, mathematical representation of the physics of the density increase due to incompressible advection of particles from a nonuniform density profile, shown in Fig. 1(b). In this case, the equilibrium density profile is flat and the actual density profile has a nonzero density gradient enforced by particle sources at the boundaries of the region of interest. The key feature that the multiple-scale model reproduces is the separation between the equilibrium and mean density profiles. It is this separation that provides the free energy for both the linear instability and for the resulting nonlinear fluctuations.

Part of the motivation for this study is the need to understand whether this evidently convenient method for modelling the background density gradient is a valid one. As will be shows in Sec. VII, the additional term can have its own nontrivial consequences. The most important is the introduction of an asymmetry between the quality of the $\boldsymbol{E} \times \boldsymbol{B}$ trapping at the potential maxima and minima. The question of whether this asymmetry is significant will be addressed in Sec. IX.

The geometry used for the simulations studied in this paper is a $2 \frac{1}{2}$-dimensional shear-free slab, meaning that one position coordinate, which we take to be $z$, is ignorable. Alternatively, when viewed in Fourier space, the $2 \frac{1}{2}$-dimensional condition implies that the only $k$ modes kept are those with $k_{z}=0$. The magnetic field is taken to be uniform and inclined to the $z$ axis, $B=B(\hat{z}+\theta \hat{y})$, where $\hat{\boldsymbol{y}}$ and $\hat{\boldsymbol{z}}$ are unit vectors in the $y$ and $z$ directions. The parallel wave number is then $k_{\|}=\theta k_{y}$. The inclination angle $\theta$ is a (usually small) constant in the shear-free slab models. Weak shear can be taken into account by making $\theta$ depend on the radial position coordinate $x$. For constant shear, $\theta=x / L_{4}$ where $L_{\mathrm{s}}$ is the shear length. This geometry is shown in Fig. 2. The boundary conditions are periodic, both for the particle recycling and for the wave-field quantities (densities and electrostatic fields).

The "2 $\frac{1}{2}$-dimensional" assumption makes the simulation essentially two-dimensional. The particle variables followed are the velocity component $v_{\|}$, the magnetic moment $\mu$, and the two spatial variables, $x$ and $y$. Apart from a saving of one spatial dimension compared with a fully three-dimensional code, the $2 \frac{1}{2}$-dimensional condition also considerably simplifies the theoretical analysis of the properties of the particle orbits, as will be seen in Sec. IV.

All Fourier modes of the electrostatic potential with $k_{x}=0$ have been set to zero in order to satisfy the assumed multiscale ordering between $k$ and $k_{x} .{ }^{2}$ All potential modes with $k_{y}=0$ except for that with the second harmonic value of $k_{x}$ have also been zeroed out. ${ }^{2}$ 


\section{PHENOMENOLOGY}

\section{A. Parameters Used}

The choice of parameters used for the simulations considered here was constrained by the requirement that the linear instability be strong enough to grow out of the thermal background noise. As has been noted by Lee, ${ }^{5}$ the thermal fluctuations can broaden the linear electron Landau resonance, so as to suppress the linear growth of the instability. For the instability to grow, it is necessary that $\eta_{\mathrm{in}} / \Omega_{\mathrm{i}} \gtrsim k_{\perp} \rho_{\mathrm{s}} /(2 \sqrt{N})$, where $\operatorname{Tin}_{\text {in }}$ is the linear growth rate. For a given particle number $N$, this results in a lower bound on the ratio $n / \theta$.

The parameter values used for the simulations ar: $n_{\mathrm{e}} / m_{\mathrm{i}}=1 / 1837, \tau \doteq T_{\mathrm{e}} / T_{\mathrm{i}}=$ 4 or $100, \rho_{\mathrm{s}} / \Delta=4.286, \lambda_{\mathrm{D}} / \Delta=1, \kappa \rho_{\mathrm{s}}=0.214$ or $0.107, L_{x}=L_{y}=32 \Delta, n_{0}=$ $16 / \Delta^{2}, \theta=0.002$ or $0.01, \nu_{\mathrm{ei}} / \Omega_{\mathrm{i}}=0,0.001$, or 0.01 , and $\left(k_{x}, k_{y}\right)=(0.842 \mathrm{~m}, 0.842 n)$, where $m$ and $n$ are integers. Here, $\Delta$ is the grid spacing (equal for $x$ and $y$ ), $L_{x}$ and $L_{y}$ are the lengths of the simulation box in the $x$ and $y$ directions, and the other symbols have been defined before. One run, for $T_{e} / T_{i}=4, \theta=0.002, \nu_{\text {ei }} / \Omega_{\mathrm{i}}=0.001$, and $\left(k_{x}, k_{y}\right)=(0.842 n, 0.21 n)$, corresponding to $L_{y}=4 L_{x}=128 \Delta$ was also made. Because of the tendency of the $E \times B$ nonlinearity to drive the fluctuations towards isotropy, the $L_{x}=L_{y}$ runs were expected to give the most spatially coherent states. The $L_{y}=4 L_{x}$ run was made in an attempt to produce a more turbulent state. Linearly, these parameters correspond to collisionless or weakly collisional drift waves, although as will become clear, the nonlinear properties of the saturated states depend strongly on the collisionality. The simulation runs typically use a timestep of $d t \simeq \Omega_{i}^{-1} \simeq 0.18 \omega_{-}^{-1}$, and were run for about 2000 timesteps. The $L_{x}=L_{y}=32 \Delta$ runs take around 2 hours of CPU time on the Cray-1.

\section{B. Linear Theory}

The linear theory of drift waves (using the number-conserving BGK collision operator), for the parameter values used here, is well known and is summarized, for example, in reference. ${ }^{2}$ We repeat the main results here, casting them in gyrokinetic form. For $\tilde{\omega} /\left(k_{\| l} v_{\mathrm{te}}\right) \ll 1$ and $\tilde{\omega} /\left(k_{\|} v_{\mathrm{ti}}\right) \gg 1$, where $\tilde{\omega}=\omega+i \nu_{\mathrm{ei}}$, the electron response is nearly adiabatic

$$
n^{e} \simeq\left(1-i \alpha_{\text {Li }}\right) n_{0} \frac{e \phi}{T_{e}},
$$

where $\alpha_{\mathrm{j}_{\mathrm{n}}}$, the phase shift associated with the nonadiabatic part, is given by

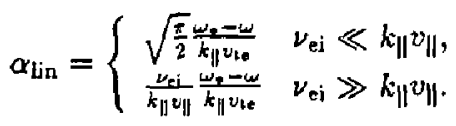


We will be working in the regime where the ions are essentially cold in the parallel direction and the Larmor radius is smaller than the perpendicular wavelength. The ion gyrocenter response is then

$$
\bar{n}^{\mathrm{i}} \simeq n_{0}(1-b) \frac{\omega_{-}}{\omega} \frac{e \phi}{T_{\mathrm{e}}},
$$

where $b \doteq k_{\perp}^{2} \rho_{\mathrm{i}}^{2} \ll 1$ and $\omega_{*} \doteq \kappa \rho_{\mathrm{s}} k_{y} \rho_{\mathrm{s}} \Omega_{\mathrm{i}}$ is the diamagnetic drift frequency. In the regime where $\lambda_{\mathrm{de}} / \rho_{\mathrm{s}} \ll 1$, and $b \ll 1$, the gyrokinetic Poisson Eq. (2) becomes

$$
k_{\perp}^{2} \phi \simeq \bar{n}^{i}-n^{e} .
$$

Inserting the gyrocenter responses gives the real frequency and growth rate, respecivively, as

$$
\frac{\omega \operatorname{lin}}{\omega_{*}}=\frac{1-b}{1+\tau b}
$$

and

$$
\frac{\gamma_{\operatorname{lin}}}{\omega_{\operatorname{lin}}}=\frac{\alpha}{1+\tau b} .
$$

The choice of parameters used in the gyrokinetic simulation runs satisfies $\omega / k_{\|} v_{t e} \sim$ $0.5-1$, but a numerical solution of the dispersion relation agrees fairly well with this calculation and gives $\left(\omega_{\text {lin }}+i \gamma_{\text {lin }}\right) / \Omega_{\mathrm{i}}= \pm 0.06+0.011 i$ for the $\theta=0.01$ cases and $\pm 0.034+0.027 i$ for the $\theta=0.002$ cases, with $\tau=4, \nu_{\mathrm{ej}}=0$, and $k_{x} \rho_{\mathrm{s}}=k_{\mathrm{y}} \rho_{\mathrm{s}}=0.842$. The effect of weak collisions on the linear behavior is small. The maximum growth rates occur for $\tau b \simeq 2 \tau /(3+\tau)$, corresponding to the lowest available modes in the $k_{x} \rho_{s}=k_{y} \rho_{s}=0.842$ cases. This choice was made deliberately, in order to produce a relatively coherent state that would have the greatest chance of being analytically tractable. The simulations with $L_{y}=128 \Delta$ yield similar values for the particle flux and saturation levels. In this run, the lcwest modes are no longer the most strongly unstable but they nevertheless have the highest saturation levels. This interesting observational result will be diseussed further in the later sections. Note also that $\omega_{\operatorname{lin}} \propto \kappa$ and $\gamma_{\operatorname{lin}} / \omega_{\operatorname{lin}} \propto \kappa / \theta$.

\section{Units}

Following the convention used, for example, in the papers of Lee and coworkers, in most of what follows the system of units used has lengths scaled to $\rho_{*}$, times scaled to $\Omega_{i}^{-1}$, densities scaled to the mean density $n_{0}$, and electrostatic potentials scaled to $T_{e} / \epsilon$, where $e$ is the unit charge. In this system of units, the electron diamagnetic frequency is $\omega_{z}=k_{y} \kappa=0.180$ for most of the cases sturlind here. 
Exceptions are runs with $k_{y}=0.21$ and $\kappa=0.214$, for which $\omega_{-}=0.045$, and runs with $\kappa=0.107$ and $k_{y}=0.842$, for which $\omega_{*}=0.09$. Thus, diffusion coefficients are normalized to $\rho_{B} c_{2}$. For compactness, we will most often still write $v_{t e}$ instead of $\sqrt{m_{\mathrm{j}} / m_{\mathrm{e}}}$.

\section{Phenomena}

We now summarize the phenomenology of the gyrokinetic particle simulation runs, the dynamics of which we explain in the subsequent sections. Our aim in this section is both to review the phenomenology of gyrokinetic simulation results for collisionless drift waves in a shear-free slak, already in the published literature, ${ }^{3,2}$ and to summarize those results from more recent simulations done by the present authors which help guide the development of the theory.

The time evolution can be divided up roughly into four stages: a pre-mode stage, a linear stage, a saturation stage, and a steady-state stage. Figure 3 shows the time history of the $(m, n)=(1,1)$ and $(1,-1)$ modes, and Fig. 4 shows the particle flux for $\theta=0.002, \tau=100$, and $\nu_{\mathrm{ei}}=0.001$. The "pre-mode" stage, for $t \simeq 0-60\left[\left(\omega_{-} t\right) /(2 \pi) \simeq 0-1.7\right]$, represents the time before the linear mode is set up and is peculiar in that the $(1,1)$ mode has phase velocity in the ion diamagnetic drift direction for part of this time. This stage has been found to be the result of the linear evolution before it has reached the time-asymptotic regime that is usually treated in the linear theory. The linear phase is the time, in this case $t \simeq 60$ $200[(\omega, t) /(2 \pi) \simeq 1.7-5.7]$, during which the simulation obeys the predictions of linear theory-i.e., a linearly growing mode, and a corresponding quasilinear particle flux. The "saturation phase" of the evolution, which may consist of more than one identifiable substage (in this case two substages, from $t \simeq 200-750$ and $t \simeq 750$ 1200), is the time during and after the saturation of the mode, before the steadystate phase. The "steady-state" phase is the final stage of the evolution, in which the mode amplitude and the particle flux have settled down to fluctuate about final, relatively steady, mean values. The term "saturated states" will be used to refer to the states observed during the saturation and steady-state phases, except where any distinetions are important.

The saturated states for the $L_{x}=L_{y}$ runs are characterized by a very strong dominance of the $(m, n)=(1,1)$ and $(1,-1)$ modes for the potentials. The density is dominated, less strongly, by the $(1,1),(1,-1)$, and the $(2,0)$ and $(0,2)$ modes (and their complex conjugates). Since we will be discussing these modes quite frequently, they will be denoted by their $n$ values-i.e., as the " + ", "-", " 0 ", and "2" modes. These will also be used as subscripts to denote the modes of the potential and the densit: .3. Tables 1 and 2 show some of the mode amplitudes of the potential and the densities in the saturated states for the same run as in Fig. 3. The mean squared amplitudes of the $\left(k_{x}, k_{y}\right)$ and $\left(k_{x},-k_{y}\right)$ modes are equal to within the 
quoted accuracy (a factor of two). The strong dominance of these modes can also be seen from the potential contour plots, such as the one in Fig. 5 which shows the potential contours at two different times in the late stages of a run with $\theta=0.002$, $\tau=100$, and $\nu_{\mathrm{ei}}=0.001$. The degree to which the lowest modes dominate the fluctuation spectrum for the $\theta=0.002$ cases is surprising since the linear growth rates fall off quite gradually with increasing $|\boldsymbol{k}|$ for this choice of $\theta$. The saturation levels of the dominant modes generally increase, although not very strongly, with $\nu_{\mathrm{ej}}$ and $\kappa / \theta$. The values range from $0.5 \%$ for the $\theta=0.01, v_{\mathrm{ci}}=0, \tau=4$ case to about $6 \%-8 \%$ for the $\nu_{\mathrm{ei}}=0.01$ cases for both values of $\theta$ used. The saturation levels are slightly increased (by a factor of about 1.3) when $\tau$ is decreased from 100 to 4 in the $\theta=0.002$ cases. The $32 \times 128$ run, for which $k_{y}$ is reduced by a factor of 4 in comparison with the $32 \times 32$ runs, has a saturation level of about $10 \%$ for the dominant $\left(k_{x}, k_{y}\right)=(0.842, \pm 0.21)$ modes.

There are several noteworthy features in the time dependence of the dominant modes. The + and - modes of both the density and the potential have a roughly sinusoidal time dependence in the saturated state. These oscillations are qualitatively similar to the linear oscllations, exccpt that they do not grow and have slightly shifted real frequencies. The mean saturation levels of $\phi_{+}$and $\phi_{-}$are the same and increase with collision frequency. The real frequencies, $\omega_{+}$and $\omega_{-}$, of $o_{+}$and $\phi_{-}$have both a mean shift relative to the linear frequency and a frequency difference for the $\theta=0.002$ collisional cases. In these cases, $\omega_{+}$is greater than (the absolute value of ) $\omega_{-}$, implying a mean motion of the whole system of $\phi$ contours in the $x$ direction-i.e., in the direction opposite to that of the density gradient. The mean real frequencies run from $\omega_{\mathrm{s}}=0.035$ to 0.05 and, unlike the values for the linear frequencies given in Eqs. (10), do not show any obvious $\theta$ dependence. The $32 \times 128$ run with $\tau=4$ and $\nu_{e i}=0.001$ has a real frequency in the saturated state of $\omega_{s}=0.015$, in agreement with the expectation that $\omega_{\mathrm{r}} \propto k_{\mathrm{y}} /\left(1+k_{\perp}^{2}\right)$ and the results of the $32 \times 32$ runs. The real frequency difference is zero for the collisionless cases (and all of the $\theta=0.01$ cases) and increases with collisionality. For $\theta=0.002$ its values are in the range of $\Delta \omega_{\mathrm{r}} \simeq 0.003$. There is a nonlinear broadening, $\delta \omega$, of the real frequencies, the value of which is given by $(\delta \omega / \omega) \sim 0.2-0.4$. This frequency broadening decreases as a function of $\nu_{\mathrm{ei}}$.

The saturation amplitudes fluctuate with time on a time scale characteristic of the $\boldsymbol{E} \times \boldsymbol{B}$-trapping time. This characteristic fluctuation frequency increases as a function of $\nu_{\mathrm{ei}}$ and decreases with $\theta$. Since the characteristic $\boldsymbol{E} \times \boldsymbol{B}$-trapping time is proportional to the saturation level, this observation is in agreement with the observed dependences of the saturation level which were noted earlier. Also. the relative fluctuations in the saturation amplitude decrease as a function of $\nu_{\mathrm{ej}}$. Finally, there is a clear negative correlation between the fluctuations in the + and mode amplitudes. Upon comparing the time histories of $\left|\phi_{+}\right|^{2}+\left|\phi_{-}\right|^{2}$ and $\left|\phi_{+}\right|^{2}$ it is seen that more than half of this fluctuation is due to an "energy exchange" between 
these two modes.

The particle flux, which is intrinsically ambipolar, is due almost entirely to the contributions from the + and - modes. In the linear phase, the flux is given by the quasilinear value. The final steady-state flux is generally observed to be much less than that expected from quasilinear theory and increases with $\nu_{\text {ei }}$ and $\kappa / \theta$. Values of the time-averaged flux in the saturated phase for various other parameter values are also marked in Fig. 4.

In the collisional $\theta=0.002$ cases, regions of enhanced electron and ion gyrocenter density are observed, as shown in Fig. 6 . These coincide in position with the maxima of the potential and will be referred to as "globs" in ihe remainder of this paper. Their rate of growth in number density increases with collision frequency.

The above phenomena remain largely unchanged if some terms are removed from the equations that are being simulated. These terms are the nonlinear terms in $\Psi$ in Eq. (1b) and in Eq. (2), and the ion parallel velocity (i.e., $v_{\| \mathbf{i}}$ set to zero). Figure 7 shows the time histories of the $\phi_{+}$mode and of the flux for such a run for $\nu_{\text {ei }}=0.001, \theta=0.002$, and $\tau=100$. The intermediate "saturation phase" is somewhat shorter in this case than for the corresponding full code run. Changing the value of $\tau$ from 100 to 4 does produce a slight increase in the saturation levels and an increase in the particle fluxes. The increase in particle fluxes is small for $\theta=0.002$ but is roughly a factor of two for the $\theta=0.01, \nu_{\mathrm{ej}}=0.01$ case. Also, studies using a partially linearized version of the particle code ${ }^{19}$ have shown that the nonlinear effects due to the electron parallel acceleration can be removed without drastically changing the behavior.

The larger $L_{y}=4 L_{x}=128 \Delta, \nu_{\mathrm{e} i}=0.001, \theta=0.002$ run has behavior very similar to the corresponding run with $L_{x}=L_{y}=32 \Delta$ considered above. It is also dominated by the $(m, n)=(1,1)$ and $(1,-1)$ modes. The saturation levels of these modes is around $10 \%$, somewhat higher than for the smaller-box-size run, but the value of the particle flux is the same. That the $(1,1)$ and $(1,-1)$ modes dominate in this case is interesting since these are not the most unstable linearly. The most unstable modes are the $(1,4)$ and $(1,-4)$ modes, which have the same values of $k_{x}$ and $k_{y}$ as the + and - modes of the $32 \times 32$ simulations. The amplitudes of these modes have been reduced by a factor of about 50 compared with their values in the $32 \times 32$ simulation. Thus the saturation levels are clearly not determined by linear considerations alone.

Summarizing the above discussion, the following phenomena have been observed:

1. Pre-linear, linear, saturation, and steady-state phases of the evolution.

2. The,,+- 0 , and 2 modes dominate the post-linear phases.

3. The + and - modes oscillate with nonlinearly modified real frequencies in the post-linear phases. The nonlinear modifications include: 
- a frequency shift,

- a frequency broadening,

- a frequency difference implying a net $x$ component of the phase velocity,

- amplitude fluctuations implying an energy exchange between the + and modes as well as a smaller fluctuation in the sum of the energies.

4. A steady-state flux which is due almost entirely to the + and - modes, has a value much less than expected from quasilinear theory, and increases with $\nu_{\mathrm{ej}}$ and decreases with $\theta$.

5. Globs - regions of enhanced particle density near the maxima of $\phi$ in the $\theta=0.002, \nu_{\text {ej }} \neq 0$ runs.

6. The nonlinear terms in $\boldsymbol{\Psi}$ and in the gyrokinetic Poisson equation and the ion parallel motion are unimportant for the steady-state phase.

\section{HAMILTONIANS FOR $2 \frac{1}{2}$-DIMENSIONAL GYRO- CENTER MOTION}

In the $2 \frac{1}{2}$-dimensional approximation, the phase space of the collisionless gyrocenter trajectories is two-dimensional in the sense that the phase-space coordinates can be taken to be the (configuration-space) variables $x$ and $y$. This fact can be exploited to show that these trajectorjes themselves are given by a two-dimensional (generally time-dependent) Hamiltonian system of equations. For time-independent electrostatic potentials, this put a severe constraint on the parijcle paths, namely tnat they cannot be chaotic. Thus, any chaos in the particle paths must be associated with a time dependence of the potential. This section gives the method for deriving the Hamiltonians as well as the results of the derivation. Most of the analysis of these Hamiltonians is left for subsequent sections.

The grocenter equations of motion used by the particle simulation, neglecting collisions, are given by Eq. (4), along with the paralle! Newton's law (in Gaussian units)

$$
\dot{v}_{\sharp}=-\frac{q}{m} \frac{\partial \Psi}{\partial z} .
$$

In the $2 \frac{1}{2}$-dimensional model, $\Psi$ is independent of $z$, and $y$ varies both due to the component of velocity perpendicular to the magnetic field and that parallel to it. Thus, $\dot{y}=v_{\mathrm{py}}+\theta \dot{z}$ where $v_{\mathrm{py}}$ is the $y$ component of $v_{\text {perp. }}$. Upon taling this into account and using $\partial / \partial z=\theta \partial / \partial y$ to express $E_{\|}$in terms of $\dot{x}$, we obtain (in scaled 
units)

$$
\begin{aligned}
\dot{x} & =-\frac{\partial \Psi}{\partial y}, \\
\dot{y} & =\frac{\partial \Psi}{\partial x}+\kappa \Psi+\theta v_{\|}-v_{\mathrm{ph}}, \\
\dot{v}_{\|} & =\left\{\begin{aligned}
-\frac{m_{\mathrm{s}}}{m_{\mathrm{e}}} \theta \dot{x} & \text { (electrons), } \\
\theta \dot{x} & \text { (ions). }
\end{aligned}\right.
\end{aligned}
$$

where a Galilean transformation to a frame moving with fixed velocity $v_{\text {ph }}$ in the $y$ direction has been made. If the waves represented by the potential $\Psi$ have a well-defined phase velocity $v_{\mathrm{ph}}=\omega_{\mathrm{r}} / k_{\mathrm{y}}$ in the $y$ direction, then this choice of $v_{\mathrm{ph}}$ represents a transformation to the wave frame and makes the resulting $\Psi$ timeindependent. This transformation will also be seen to preserve the Hamiltonian nature of these equations. Within the framework of these equations, it is possible to generalize $\theta$ to include the effect of weak shear and to generalize $\kappa$ to include the effect of a nonzero temperature gradient. For the case of weak shear, the form $\theta=x / L_{\mathrm{a}}$ is taken, where $L_{\mathrm{s}}$ is the shear length. Since shear tends to localize the eigenmodes around the rational surface, it is convenient in the models with shear to place the rational surface at $x=0$, so that $\theta$ has no constant term. Thus, we consider four main classes of models:

- without shear, $\theta=$ constant,

- with shear, $\theta=x / L_{\mathrm{s}}$,

- without temperature gradient, $\kappa=$ constant,

- with temperature gradient, $\kappa=\kappa_{n}\left[1+\eta_{j}\left(\alpha v_{\|}^{2}-\frac{1}{2}\right)\right]$,

where

$$
\alpha= \begin{cases}m_{\mathrm{e}} / m_{\mathrm{i}} & \text { (ejectrons), } \\ \tau & \text { (ions), }\end{cases}
$$

and the subscript $j=(e, i)$ denotes the particle specjes. Here $L_{s}$ is the shear length, $\eta \doteq\left(d \ln T_{0} / d \ln n_{0}\right)$, and $x_{n} \doteq-d \ln n_{0}(x) / d x$. Here, $n_{0}(x)$ is the background density and $T_{0}(x)$ is the background temperature. The notation has been slightly changed in this section, so that $\kappa$ now denotes the total logarithmic pressure gradient, including the temperature gradient. For $\eta=0$, the subscript will be dropped so that $\kappa$ will appear instead of $\kappa_{n}$.

The procedure for deriving the Hamiltonians is as follows. First, Eq. (11c) is integrated with respect to time to yield a constant of motion which is a function 
of $x$ and $v_{\|}$. We define

$$
\zeta=\left\{\begin{aligned}
-\frac{m_{1}}{m_{4}} \theta & \text { (electrons, no shear) } \\
\theta & \text { (ions, no shear) } \\
-\frac{m_{1}}{m_{e} L_{2}} & \text { (electrons, with shear) } \\
\frac{1}{L_{*}} & \text { (ions, with shear) }
\end{aligned}\right.
$$

The constant of motion is then

$$
C_{1}= \begin{cases}v_{\|}-\zeta x & \text { (shear-free) } \\ v_{\|}-\frac{1}{2} \zeta x^{2} & \text { (with shear) }\end{cases}
$$

This constant of motion is then used to eliminate either $x$ or $v_{\|}$from Eqs. (IIa) and (11b). If the parallel-trapping frequency is small or comparable to the $\boldsymbol{E} \times \boldsymbol{B}$ trapping frequency, then it is more physical to eliminate $v_{\|}$. An integrating factor $I(x)$ for $\mathrm{Eq}$. (11b) can be found from the $(\partial \Psi / \partial x)$ term and the $\kappa \Psi$ terms. For $\eta=0$, we have $I(x)=\exp (\kappa x)$. For the cases with nonzero $\eta$,

$$
I(x)= \begin{cases}\exp \left\{\kappa_{n}\left(x+\eta_{j}\left[\frac{\alpha}{3 \zeta}\left(\zeta x+C_{1}\right)^{3}-\frac{1}{2} x\right]\right)\right\} & \text { (no shear), } \\ \exp \left\{\kappa_{n}\left(x+\eta_{j}\left[\alpha\left(\frac{1}{20} \zeta^{2} x^{5}+\frac{1}{3} \zeta C_{1}+C_{1}^{2} x\right)-\frac{1}{2} x\right]\right)\right\} & \text { (with shear). }\end{cases}
$$

Now Eq. (1Ib) can be rewritten as

$$
\dot{y}=\frac{1}{I(x)} \frac{\partial}{\partial x}[I(x) \Psi]+V(x)
$$

where

$$
V(x)= \begin{cases}\zeta \theta x+C_{1} \theta-v_{\mathrm{ph}} & \text { (no shear) } \\ \frac{\zeta}{2 L_{\mathrm{a}}} x^{3}+\frac{C_{\perp}}{L_{\mathrm{a}}} x-v_{\mathrm{ph}} & \text { (with shear) }\end{cases}
$$

The equations for $\dot{x}$ and $\dot{y}$ can thus be written in the Hamiltonian form

$$
\begin{aligned}
& \frac{d x}{d s}=-\frac{\partial}{\partial y} H \\
& \frac{d y}{d s}=\frac{\partial}{\partial x} H
\end{aligned}
$$

where

$$
\frac{d}{d s} \doteq I(x) \frac{d}{d t}
$$

and

$$
H=I(x) \Psi(x)+\int^{x} d x^{\prime} I\left(x^{\prime}\right) V\left(x^{\prime}\right)
$$


A few comments about the above procedure are in order. This procedure takes the parallel acceleration into account for nonzero $\zeta$. When $v_{\|}$is eliminated using Eq. (13), the parallel velocity is expressed as a function of $x$. The terms representing the effects of the parallel acceleration are those containing nonzero powers of $\zeta$. The transformation to the new "time" variable $s$ is necessitated because, along their trajectories, the particles move more slowly for higher values of $I(x)$. Thus, the $x-y$ area element containing a group of gyrocenters is compressed by a factor $I\left(x_{2}\right) / I\left(x_{1}\right)$ as it travels from a position with $x$ coordinate $x_{1}$ to one with $x$ coordinate $x_{2}$. In the simplest case with $\eta=0$, the compression models the physical effect of the incompressible advection by the $\boldsymbol{E} \times \boldsymbol{B}$ drift of a background density with the spatial profile $n_{0} \exp (-\kappa x)$. For nonzero $\eta$ and zero parallel acceleration $(\zeta=0)$, $I(x)$ also contains the effect of the $E \times B$ advection of gyrocenters taken from a Maxwellian with a background temperature profile $T_{0} \exp \left(-\kappa_{n} \eta x\right)$. When parallel acceleration is taken into account $(\zeta \neq 0)$, the $E \times B$ advection is accompanied by a parallel acceleration which can be regarded as an advection in velocity space. 1 ais causes a change in the distribution function, since the background distribution function which is being advected in velocity space is nonuniform. (In addition to the phase space compression effects, the multiscale model also modifies the $\boldsymbol{E} \times \boldsymbol{B}$ trapping when the electrostatic potential is time-dependent. The significance of this change will be examined later.)

It is straightforward to write the Hamiltonian of Eq. (14) in closed form for the cases with $\eta_{j}=0$. The results are

$$
H= \begin{cases}e^{\kappa x}\left\{\Psi+\frac{\zeta \theta}{\kappa} x+\frac{1}{\kappa^{2}}\left[\left(\theta C_{1}-v_{\mathrm{ph}}\right) \kappa-\zeta \theta\right]\right\} & \text { (no shear), } \\ e^{\kappa x}\left\{\Psi+\frac{\kappa}{2 L_{0} \kappa^{2}}\left(\kappa x^{3}-3 x^{2}\right)+\frac{x}{\kappa L_{*}}\left(C_{\mathrm{l}}+\frac{3 \zeta}{\kappa^{2}}\right)-\frac{1}{\kappa}\left(\frac{3 \zeta}{L_{0} \kappa^{3}}+\frac{C_{1}}{L_{\mathrm{l}} \kappa}+v_{\mathrm{ph}}\right)\right\} & \text { (sheared). }\end{cases}
$$

For the ions, particularly in the case of the collisionless drift mode in a shear-free slab, the limit of zero parallel velocity is of interest. The result is

$$
H=e^{\kappa x}\left(\Psi-\frac{v_{p h}}{\kappa}\right) \text {. }
$$

The $\kappa=0$ limit of this result has been either the focus of, or the starting point for, several analyses of the nonlinear effects of drift waves on gyrocenter orbits. ${ }^{14-17}$ For later use we summarize the $\kappa=0$ results, including the other cases with parallel acceleration and with shear. The results are

$$
H= \begin{cases}\Psi-v_{\mathrm{ph}} x & \left(v_{\|}=0\right) \\ \Psi+\frac{1}{2} \theta \zeta x^{2}+\left(\theta C_{1}-v_{\mathrm{ph}}\right) x & \text { (no shear) } \\ \Psi+\frac{\zeta}{8 L_{0}} x^{4}+\frac{C_{1}}{2 L_{\mathrm{o}}} x^{2}-v_{\mathrm{ph}} x & \text { (with shear) }\end{cases}
$$

The first of these is the one given by Ching. ${ }^{14}$ Note that the Hamiltonians of Eq. (15) contain constants which approach $\infty$ as $\kappa \rightarrow 0$. These constants, however, are easily 
identified (as the constants inside the expressions multiplied by the exponential) and subtracted off, if this is desired. The limit of zero parallel acceleration can be obtained by setting $\zeta=0$ and $C_{1} \rightarrow v_{\|}=$const. in any of the above results. Thus, for example, the Hamiltonian for free-streaming electrons in a shear-free magnetic field of Smith et al. ${ }^{18}$

$$
H=\Psi+\left(\theta v_{\|}-v_{\mathrm{ph}}\right)
$$

follows from the second result of Eq. (17).

The above Hamiltonians are of greatest utility when, by a suitable choice of $v_{\mathrm{ph}}$, $\Psi$ can be made independent of time. The gyrocenter trajectories are then given by two-dimensional time-independent Hamiltonians and are, therefore, integrable. These Hamiltonians are also of use in time-dependent cases since the instantaneous streamlines of the flow field in phase space are contours of constant $H$ and $C_{1}$. The existence of a Hamiltonian and its particular form greatly simplifies the determination of the qualitative be javior of the gyrocenter orbits.

\section{TEST ION AND ELECTRON GYROCENTER SIMU- LATION}

\section{A. Simulation Method}

Motivated by the observation given in Sec. III, that the saturated $\phi$ spectra are dominated by the + and - modes and that these modes fluctuate with a clear negative correlation [that is, the correlation function of the mode amplitudes $\left.\left(\left|\phi_{+}\right|-\overline{\left|\phi_{+}\right|}\right)\left(\left|\phi_{-}\right|-\overline{\left|\phi_{-}\right|}\right)<0\right]$, the following model was used to study the physics of the non-self-consistent rootion of the ion and electron gyrocenters. For the ions, the parallel motion and the nonlinear terms in $\Psi$ are neglected, while for the electrons the gyroradius is neglected. Thus we use

$$
\Psi= \begin{cases}\bar{\phi}=\phi(1-b) & \text { ions, } \\ \phi & \text { electrons. }\end{cases}
$$

For the electrons, the equations of motion include those for the parallel motion, the parallel acceleration, and the pitch-angle scattering. The collision model used is the Monte-Carlo pitch-angle scattering model of Boozer et al. ${ }^{27}$ For the electrostatic potential $\phi$ only the + and - modes are kept. The amplitudes $\phi_{+}$and $\phi_{-}$are allowed to tluciuate with time, the mean values of the amplitudes being taken to be the same and with the time dependence of the two modes negatively correlated. For simplicity, the time dependence of the two modes is takein to be quasiperiodic with two frequency components. Although the actual time dependence of the mode amplitudes in the simulations is chatic, the nonlinearity of the gyrocenter motion 
will significantly reduce any spurious correlations between the time dependence of the mode amplitudes and the gyrocenter motion. The $\phi$ modes are taken to propagate in the $y$ direction with phase velocity $v_{\mathrm{ph}}$ and in the $x$ direction with velocity $v_{x}$. Thus, the resulting electrostatic potential is

$$
\begin{aligned}
\phi= & -2 \phi_{+}(t) \cos \left[k_{x}\left(x-v_{x} t\right)+k_{y}\left(y-v_{\mathrm{ph}} t\right)\right] \\
& +2 \phi_{-}(t) \cos \left[k_{x}\left(x-v_{x} t\right)-k_{y}\left(y-v_{\mathrm{ph}} t\right)\right], \\
= & -2 \phi_{+}(t) \cos \left[k_{x} x+k_{y} y-\omega_{+} t\right] \\
& +2 \phi_{-}(t) \cos \left[k_{x} x-k_{y} y+\omega_{-} t\right],
\end{aligned}
$$

where

$$
\phi_{ \pm}(t) \doteq \phi_{0}\left(1 \pm \epsilon_{1} \sin \omega_{1} t \pm \epsilon_{2} \sin \omega_{2} t\right) .
$$

Physically, $v_{\mathrm{ph}}$ and $v_{\mathbf{x}}$ are the mean phase velocities of the waves, respectively, in the $y$ and $x$ directions, $\phi_{0}$ is the mean amplitude of the + and - modes, and $\epsilon_{1}$ and $\epsilon_{2}$ are the amplitudes of the $\omega_{1}$ and $\omega_{2}$ frequency components of the fluctuations in the mode amplitudes. The constant parameters $k_{x}, k_{y}, v_{x}, v_{\mathrm{ph}}$ (or alternatively, $\omega_{+}$and $\left.\omega_{-}\right), \phi_{0}, \epsilon_{1}, \epsilon_{2}, \omega_{1}$, and $\omega_{2}$ are chosen to fit the data from particular runs of the gyrokinetic code, discussed in Sec. III. Note that throughout this paper, " $\phi_{0}$ " will be used to refer either precisely to the definition in Eq. (19b), in the case of references to test-gyrocenter simulation results, and to the rms value of $\phi_{+}$ or $\phi_{-}$, when results from the fully seif-consistent gyrokinetic simulations are being discussed (- and not to the 0 mode of $\phi$ ).

A computer program was written to follow the motion of a large number of test gyrocenters moving under the effect of the velocity fields given by Eqs. (11) with the potential $\Psi$ given by Eq. (18). The code was checked for agreement with the known analytical solution for $\kappa=0$. The number of guiding centers used was typically 16000 , comparable to the original gyrokinetic code. The gyrocenters are loaded pseudorandomly with a distribution which is uniform in $x$ and $y$ and Maxwellian in $v^{\prime} \|$. The motivation for using this non-self-consistent code is as follows. Firstly, the electrostatic potential has similar features in all of the gyrokinetic simulations and there is much to be learned from the study of the particle motion in such potentials. The ability to vary the parameters in the non-self-consistent code slightly away from the values observed in the full code runs enables a greater insight to be obtained into the ba' ances that determine the full self-consistent behavior. Secondly, by avoiding the need to interpolate the particle densities on a grid for the calculation of the self-consistent fields, the run time of the non-self-consistent code is of order one-tenth of that of the full code. Thirdly, in the non-self-consistent code one can selectively sample part.s of the single-particle phase space. This is a particularly useful feature for unravelling the electron dynamics and would be impossible in the full code where the particles must sample all of phase space in order to determine the self-consistent fields correctly. 


\section{B. Self-Consistency}

In this section, we discuss how the results for the particle fluxes from the non-selfconsistent simulations can be used to approximately predict the saturation levels reached in the full gyrokinetic simulations. The ion nonlinearity has to be involved in the saturation process if, in the saturated state, there is a nonzero electron (and hence, by ambipolarity, ion) flux. This follows from the form of the linearized ion gyrocenter fluid response $\bar{n}^{i}=\left(\omega_{*} / \omega\right)(1-b) \phi$ of Eq. (8) which permits no flux without wave growth. The nonlinear jon fluid equation will be examined to show how the balance of the ronlinearly generated contributions to the particle fluxes can lead to a well-defined saturation level. The non-self-consistent particle simulations provide a means for estimating thes nonlinear contributions to the particle fluxes. We argue, furthermore, that the resulting saturated states can be stable in a timeaveraged sense.

Consider the ion continuity equation obtained by integrating the gyrokinetic Vlasov equation, Ea (1), over $v_{\|}$and $\mu$ (assuming that the ions are Maxwellian in the perpendicuiar velocity space), and neglecting the parallel current:

$$
\frac{\partial \bar{n}^{\mathrm{i}}}{\partial t}+\boldsymbol{v}_{E B} \cdot \nabla_{\perp} \bar{n}^{\mathrm{i}}+\kappa \frac{\partial}{\partial y}\left(\Psi \bar{n}^{\mathrm{i}}\right)=0
$$

where $v_{E B}=\hat{z} \times \nabla_{\perp} \Psi$ and $\Psi=(1-b) \phi$. Now take the time average over the rapid $E \times B$-fluctuation time scales, in the following manner. Assume that, as is the case for the results of the simulations, there are well-defined wave frames for the + and - modes. These are defined in a multiple time scale sense from the time histories of the respective modes of $\phi, \bar{n}^{i}$, or $n^{e}$ as follows. As observed in Sec. III, the time history of $\phi_{+}$(for example) has steady, roughly sinusoidal oscillations in the saturated stages as well as in the linear phase. The real frequency of these (averaged over several oscillations, say) divided by $k_{y}$ defines a phase velocity and a resulting translation to a mean wave frame. The remaining time dependence consists of the (assumed slow) growth of the wave, the variations in the real frequency (which, if assumed slow, can be taken into account via an eikonal representation), and rapid variations with zero mean in the wave amplitude (and possibly wave phase) over which the average is to be performed. To perform the averaging, the + and mudal components of Eq. (20) are transformed to their respective wave frames, time-averaged over a time interval of order of a several oscillations $\left(\omega_{*}^{-1}\right)$, and then transformed back to the laboratory frame. When this averaging procedure has been carried out, the result can be written schematically as

$$
\left(\gamma \mp i \omega_{r \pm}\right)\left\langle 1 \mp i \alpha_{i}\right) \xi \phi_{ \pm} \pm i \omega_{-}(1-b) \phi_{ \pm}+\left(\eta \pm i \delta_{ \pm}\right) \phi_{ \pm}=0 .
$$

Here, $\gamma$ is the (assumed small) growth rate, $\omega_{x \pm}$ are the real frequencies of the + and - modes, and the ion density has been written as

$$
\bar{n}_{ \pm}^{i}=\left(1 \mp i \alpha_{i}\right) \xi \phi_{ \pm}
$$


where $\alpha_{i}$ and $\xi$ are real nonlinear coefficients. The first term is the time derivative of the ion gyrocenter density. The second term of Eq. (21) represents the linear $\boldsymbol{E} \times \boldsymbol{B}$ advection of the background density profile and results in the response of Eq. (8). The third term is the nonlinear term written with real nonlinear coefficients $\eta$ and $\delta_{ \pm}$defined by dividing the sum of all of the terms resulting from averaging the nonlinear terms of Eq. (20) by $\phi_{+}$or $\phi_{-}$, as appropriate. These coefficients will shortly be shown to be associated, respectively, with the nonlinear ion flux (both advective and diffusive) and the nonlinear frequency shift. Asymmetry between the effects of tìe + and - modes has no obvious time-averaged effect in the simulations. other than the presence of a difference between the real frequencies of the + and modes. Other effects of this asymmetry have been neglected. Now suppose that the electrons are nearly adiabatic, with a linearly or nonlinearly generated phase shift with respect to the potential, $n_{ \pm}^{t} \simeq\left(1 \mp i \alpha_{e}\right) \phi_{ \pm}$. Equation (9) then gives $\xi \simeq 1+k_{\perp}^{2}$ and $\alpha_{\mathrm{i}}=\alpha_{\mathrm{e}} / \xi$. The real and imaginary parts of the coefficients of $\phi$ in Eq. (21), respectiveiy, give

$$
\begin{aligned}
\gamma & =\omega_{\mathrm{r} \pm} \alpha_{i}-\eta / \xi, \\
\omega_{\mathrm{r} \neq} & =\frac{1}{\xi}\left[\omega_{-}(1-b)+\delta_{ \pm}\right]-a_{i} \gamma .
\end{aligned}
$$

Saturation occurs if $\gamma=0$ or equivalently if $\eta=\omega_{r \pm} \xi \alpha_{i}=\omega_{r \pm} \alpha_{e}$. Thus, there are basically three ways in which the waves can saturate. The first involves the electrons and occurs if $\alpha_{e}$ (and hence $\alpha_{i}$ ) goes to zero, for example by the threemode saturation mechanism of Lee et al. ${ }^{3}$ and Smith et al. ${ }^{18}$ The second and third saturation mechanisms relate to the ion dynamics. The second one occurs if $i_{r}$ approaches zero because of large Doppler shifts, represented by $\delta_{ \pm}$. Such large Doppler shifts are not observed in the runs considered here, but may be relevant in cases in which $\phi$ modes with $k_{y}=0$ and low $k_{x}$ are kept, as they were in the bounded code suns of Lee, Kuo, and Okuda. ${ }^{26}$ The third basic saturation mechanism involves an increase of $\eta$, so that it cancels the $\omega_{r}$ term in Eq. (23a). The nonlinear coefficient $\eta$ can be related to the ion flux as follows. By inserting Eq. (22) into the equation $\Gamma_{\mathrm{j}}=\left\langle\bar{n}^{i} V_{E_{x}}\right\rangle$ for the flux, where $V_{E_{x}}$ is the $x$ component of the $E \times B$ velocity, one obtains

$$
\Gamma_{\mathrm{i}}=2 k_{\mathrm{y}} \xi \alpha_{\mathrm{i}}\left(\left|\phi_{+}\right|^{2}+\left|\phi_{-}\right|^{2}\right) .
$$

Cpon eliminating $\alpha_{i}$ by using Eq. (23a), the following equation is obtained:

$$
\Gamma_{i}=\Gamma_{r, j}+\Gamma_{\mathrm{rl}, \mathrm{i}, \mathrm{i}}
$$

where

$$
\begin{aligned}
& \Gamma_{r, i}=2 k_{y} \frac{\gamma \xi}{\omega_{r}}\left(\left|\theta_{+}\right|^{2}+\left|o_{-}\right|^{2}\right), \\
& \Gamma_{n i, j}=2 k_{y} \frac{\eta}{\psi_{r}}\left(\left|o_{+}\right|^{2}+\left|o_{-}\right|^{2}\right)
\end{aligned}
$$


and where we have taken $\omega_{\mathrm{r}+}=\omega_{\mathrm{r}-}=\omega_{\mathrm{r}}$.

The nonlinear growth rate $\gamma$ can be related to the fluxes obtained from the nonself-consistent simulations by eliminating $\eta$ from Eqs. (23a) and (24c) to obtain

$$
\gamma=\frac{\omega_{r}}{2 k_{y} \xi}\left(\frac{\Gamma_{e}-\Gamma_{n l, i}}{\left|\phi_{+}\right|^{2}+\left|\phi_{-}\right|^{2}}\right) .
$$

This formula can be interpreted as saying that the growth rate vanishes when the nonlinear contribution to the ion flux $\Gamma_{\text {nd, }}$ balances the electron flux. This picture of the balances resulting in saturation is essentially the same as that of Dupree, ${ }^{9}$ with an additional input consisting of the knowledge that the saturated spectra are dominated by the + and - modes.

If the amplitude of $\phi$ is varied, keeping the form of its time dependence fixed, then Eq. (25) implies that there is a (nonlinearly) stable saturated state at $|\phi|=\phi_{0}$, if $\Gamma_{\mathrm{n}, \mathrm{i}}\left(\phi_{0}\right)=\Gamma_{\mathrm{e}}\left(\phi_{0}\right)$ and $d \Gamma_{\mathrm{n}, \mathrm{i}, \mathrm{d}} / d \phi_{0}>d \Gamma_{\mathrm{e}} / d \phi_{0}$. Figure 8 shows schematically the dependence of $\Gamma_{e}$ and $\Gamma_{n l, i}$ on $|\phi|$ (the different $\Gamma_{e}$ curves are for different values of other parameters, such as $\nu_{e i}$ ) and their intersections which can result in saturated states. The physics of the particle fluxes, which gives them a dependence on the amplitude of $\phi$ that enables them to balance, will be addressed in detail in the subsequent sections. $\Gamma_{e}$ can be expected to be independent of $\gamma$ if $\gamma \ll k_{\eta} v_{\text {re }}$. To show that $\Gamma_{\text {nl, }}$ is independent of $\gamma$ is difficult in general, but is straightforward in the limit where the $\boldsymbol{E} \times \boldsymbol{B}$ velocity is much less than the diamagnetic velocity. Upon assuming that $n(t)=e^{\gamma t} \hat{n}(t)$ and $\phi(t)=e^{\gamma t} \hat{\phi}(t)$, where $\hat{n}(t)$ and $\hat{\phi}(t)$ have steady Ims values, and ordering $\omega / \omega, \sim \phi \sim \gamma / \omega \sim \epsilon \ll 1$, we have

$$
\begin{aligned}
& \frac{\partial}{\partial t} \hat{n}^{(0)}=-i \omega_{\cdot}(1-b) \hat{\phi} \\
& \frac{\partial}{\partial t} \hat{n}^{(1)}=-v_{E B} \cdot \nabla \hat{n}^{(0)}-\gamma \bar{n}^{(0)} .
\end{aligned}
$$

The ion flux comes from $\hat{n}^{(1)}$ and is thus a sum of two contributions. The first of these is independent of $\gamma$ and can be identified as $\Gamma_{\mathrm{nl}, \mathrm{i}}$, while the second is $\Gamma_{\gamma, \mathrm{i}}$ of Eq. (24b). Thus, at least for $\omega / \omega$. $\ll 1$ we have shown the plausibility of the existence of stable saturated states, the saturation levels of which are given by the balance of the particle fluxes measured from the non-seif-consistent particle simulations.

The model outlined here can be regarded as an extension of that due to Dupree to a more coherent case with $2 \frac{1}{2}$-dimensional geometry. In the case studied by Dupree, the electron resonance was assumed to be unaltered by the drift waves. His justification is valid for a fully turbulent case where coherent effects are unimportant and the fluctuation spectrum is genuinely three-dimensional. Here, however. the electron resonance is modified by the parallel acceleration because of the twodimensionality of the motion. Also, the coherence of the drift waves stidied here, 
embodied in the dominance of a few modes and a small value of $\epsilon_{\max } \doteq \max |\epsilon|$, where

$$
\epsilon \doteq \frac{\phi_{+}-\phi_{-}}{\phi_{1 !}+\phi_{-}}
$$

means that even for values of $|\phi|$ for which $E \times B$ trapping occurs, the resonant ion diffusion specifically considered by Dupree may not be effective. The situation here also differs from the picture of Ching, ${ }^{14}$ in which the ion Landau damping due to the ion parallel acceleration was enhanced by the nonlinear $E \times B$ trapping. In the simulations studied here, the ion parallel acceleration is too weak to provide the Landau damping to saturate the dominant modes. The actual saturation mechanism is the $\boldsymbol{E} \times \boldsymbol{B}$ diffusion of the ion polarization density (zote that Dupree does not distinguish between the two when he refers to "Landau damping"). The potential used by Ching is the $\epsilon=0$ limit of that used here, for which there is no ion $\boldsymbol{E} \times \boldsymbol{B}$ diffusion.

\section{NON-SELF-CONSISTENT SIMULATION RESULTS}

\section{A. Ions}

We now give the results from the non-self-consistent ion gyrocenter simulations introduced in Sec. V. The equations of motion are Eqs. (11a) and (11b) ( $v_{\|}$can be set to zero) along with the potential of Eqs. (18) and (19). Figure 9 shows the time history of $\phi_{+}(t)$ for parameters chosen to model a $\nu_{\mathrm{ei}}=0.001, \theta=0.002, \tau=100$ run of the gyrokinetic code. These parameter values are $\phi_{0}=0.068, \epsilon_{1}=0.103$, $\epsilon_{2}=0.125, \omega_{1}=0.18, \omega_{2}=0.078, k_{x}=k_{y}=0.842$, and $k_{y} v_{\mathrm{ph}}=0.048$. The parameters that specify $\phi_{+}$were chosen so that the logarithm of the square of the curve in Fig. 3 qualitatively approximates the curve in Fig. 9. Scatter plots of the ion gyrocenters at $t=2000$ for these parameter values, one with $k_{x} v_{x}=0.0036$. the other with $k_{x} v_{x}=0$, are shown in Figs. 10 and 11. Note the presence of the enhanced density regions, the more prominent of which occur at the maxima of $\phi$, in Fig. 10 and their absence in Fig. 11 for which $v_{x}=0$. There is a noticeable but slight density nonuniformity in Fig. 11 near the separatrices of $\phi$ which arises because of a slight numerical inaccuracy in the timestepping. The gyrocenter flux for these cases is shown in Figs. 12 and 13. The flux is greater in Fig. 12 because in this case there is an extra contribution due to the advection of the globs. In the $k_{x} v_{x}=0.0036$ case, both the advective and diffusive components are present, while in the $k_{x} v_{x}=0$ case there is no advective component. There are globs both at the potential maxima, at $\left(\frac{x}{2 \pi}, \frac{\gamma}{2 \pi}\right)=(0.75,0.75)$ and $(0.25,0.25)$, and at the minima at $(0.75,0.25)$ and $(0.25,0.75)$. The globs at the minima are weaker than those at the maxima, for reasons which will be explained in Sec. VII. This asymmetry can be enhanced by increasing the level of the energy exchange $\epsilon$, or by decreasing the 
saturation level $\phi_{0}$. Figure 14 shows the ion gyrocenter density for a case in whic: the input parameters are the same as for Fig. 10, except that $\phi_{0}$ has been halved. This choice of input parameters corresponds roughly to the observed $\phi_{+}$and $\phi_{-}$in a sun of the gyrokinetic particle code with $\theta=0.002, \nu_{\mathrm{ei}}=0.001$, and $\tau=4$.

\section{B. Electrons}

The non-self-consistent gyrocenter code was also used to study the combined effects of the time dependence of the potential, the parallel streaming and acceleration, the collisions, and the $\kappa$ term on the electron motion. For most of the studies, the parameters of the potential were chosen as before to match a gyrokinetic run with $\theta=0.002, \nu_{\mathrm{ei}}=0.001$, and $\tau=100$, for which the time-averaged flux in the steady-state phase was $\Gamma_{e}=0.0015$. The input parameters were $\nu_{e i}=0.001$, $\theta=0.002$ (these parameters now enter explicitly into the test gyrocenter simulation), and the other parameters as given in the discussion of Fig. 9 in the previous subsection. For convenience, we call this run the "base case." Exceptions to these parameter values were made by altering one or more parameters in turn on a caseby-case basis. The most valuable information comes from the time-averaged particle flux for different settings of these parameters. Since the electrostatic potential here consists entirely of the + and - modes, the particle flux gives a direct measure of the time-averaged phase shift between those modes of the electron density and the potential (as it was in the case of the full code, since there the flux was known empirically to come almost entirely from these modes).

\section{Examination of a Single Run}

To give the reader some familiarity with the results and to show the qualitative similarities between them and those of the gysokinetic code, results for the base case from several diagnostics are shown. Figures 15-18 show, respectively, a scatter plot, the spatially averaged velocity distribution at $t=2000$, as well as time histories of the electron flux and the parallel momentum. Note that the scatter plots show enhanced density regions as were also seen for the ions. The velocity distribution function shows some depletion at the resonant velocity $\left(v_{\|}=+\omega_{r} / k_{\|}\right)$, and the averaged parallel momentum shows a steady decrease until it levels off when collisions come into play. The time average of the particle flux, $\Gamma_{\varepsilon} \simeq 0.0015$, is in reasonable agreement with the full $\mathrm{g}$;rokinetic code result. The particle flux time histories shown include both the raw trace and an averaged trace in which averages are taken over bins of $\Delta t=100$. The large relative fluctuations in the particle flux were also seen in the full gyrokinetic code results in Sec. III. 


\section{Main Results from Time Histories of the Particle Flux}

Qualitatively, the time histories of the particle fluxes for the various cases look similar to Fig. 17. The main variation bet them is in the value of the timeaveraged fluxes in the later stages of the runs. The main results involving observations of the particle flux time histories are as follows.

- With $\omega_{+}=\omega_{-}$and $\epsilon_{1}=\epsilon_{2}=0$, so that there is no energy exchange and no $x$ velocity of the waves, but with the other parameters as above, the timeaveraged flux drops to less than 0.0003 by $t=100$ and fluctuates about an essentially zero average for the rest of the run. As will le shown, this is because in this case, the particle trajectories are integrable and there is no diffusion.

- With the parallel acceleration switched off and $\omega_{+}=\omega_{-}$, and the other parameters as for the base case, $\Gamma \simeq 0.002$ (compared with $\Gamma=0.0012$ for the first case described) independent of the value of $\nu_{\mathrm{e}}$.

- Keeping the parallel acceleration off, but putting all the electrons at the resonant parallel velocity gives a time-averaged flux of $\Gamma_{e}=0.007$, suggesting that most of the particle flux comes from the resonant electrons.

The last two results, when combined with the base case run, suggest that the parallel acceleration inhibits the particle flux by depopulating the resonant electron population. The mechanism by which this can occur is as follows. In the $2 \frac{1}{2}$ dimensional model the parallel acceleration and the $\boldsymbol{x}$ component of the $\boldsymbol{E} \times \boldsymbol{B}$ drift are connected by $v_{\|}+m_{\mathrm{i}} / m_{\mathrm{e}} \theta x=$ const. Since the resonant electrons undergo the greatest motion in the $x$ direction, these also have the greatest change in parallel velocity. Thus, there is a selective depopulation of the resonance. This is best seen from the velocity distribution (at $t=2000$ ) for a $\nu_{\mathrm{ej}}=0$ run, shown in Fig. 19. This figure shows somewhat greater depletion at velocities slightly above resonance than Fig. 16 which is for $\nu_{e i}=0.001$.

- When the $x$ motion of the waves is set to zero (i.e., $\omega_{+}=\omega_{-}$), but the other parameters are left as for the base case of the previous subsection, the timeaveraged flux decreases only slightly to $\Gamma_{e}=0.001$. The time history of the parallel momentum for this case is shown in Fig. 20 which is to be compared with Fig. 18.

- The collisionless $\left(\nu_{\mathrm{ei}}=0\right)$ cases with $\omega_{+}-\omega_{-}=0$ and 0.0072 give timeaveraged particle fluxes in the steady-state phases of $\Gamma_{e}=0.3004$ and 0.001 respectively. 
For $\nu_{\mathrm{ei}}=0$ the presence of the $x$ motion of the waves thus has a much larger effect. The flux for the fun with $\nu_{e i}=0$, and $\omega_{+}=\omega_{-}$seems to be still decreasing at $t=2000$. The collisions thus enhance the particle flux in the $\omega_{+}=\omega_{-}$case. They do this by causing repopulation of the resonant region of velocity space against the depletion caused by the parallel acceleration.

To summarize, in the $\nu_{\mathrm{ei}}=0.001, \theta=0.002$ run of the gyrokinetic code, the electron flux has both a diffusive and advective component. The diffusive component of the particle flux is due mostly to the $E \times B$ diffusion of resonant electrons. The diffusion is caused by the time-dependent energy exchange between the + and - modes of the electrostatic potential. Perhaps the most important new result from these non-self-consistent runs is that the parallel acceleration can inhibit the diffusion by accelerating the initially resonant electrons away from resonance, while the collisions repopulate the resonance. When collisions are present, the decrease in the flux when the motion of the waves in the $x$ direction is switched off is quite small because the diffusive flux increases. Thus, for a given mean electrostatic potential and time dependence of the energy exchange between the + and - modes, the diffusive electron gyrocenter flux sets a lower bound on, and the approximate level of, the particle flux.

A run with input parameters chosen to match the $\theta=0.01, \nu_{\mathrm{ei}}=0.01, \tau=4$ run gives $\Gamma_{e}=0.0006$, in good agreement with the gyrokinetic code results.

The good agreement between the non-self-consistent code results and those from the full code indicates that although the actual potential in the gyrokinetic code runs is a result of self-consistent effects, the model used in the non-self-consistent code is adequate to give the electron flux. In particular, consideration of the effects of higher modes of the potential on the electron motion is unnecessary, and there are no special correlations between the time dependence of the electrostatic potential and the electron density enforced by self-consistency that need to be taken into account in order to explain the electron gyrocenter flux. Such correlations are, however, needed to account for the absence of globs near the potential minima in the runs of the full gyrokinetic code.

\section{INSTANTANEOUS VELOCITY STREAMLINES}

\section{A. Ions}

The instantaneous velocity streamlines at time $t_{0}$, say, can be defined as the trajectories that the particles would take if they moved under the influence of the potential $\phi(x, y, t)$ frozen at $t_{0}$, that is, under the influence of $\phi\left(x, y, t_{0}\right)$. The formation of the regions of enhanced density can be understood in terms of the instantaneous velocity streamlines for the gyrocenters in the $x-y$ plane. We begin 
by examining the instantaneous ion velocity streamlines. The expression for $\phi$ of Eq. (19a), combined with the zero parallel velocity limit of Eqs. (11a) and (11b), yields

$$
\begin{aligned}
\dot{X}= & -2\left[\hat{\psi}_{+} \sin (X+Y)+\hat{\psi}_{-} \sin (X-Y)\right]-U_{x} \\
\dot{Y}= & 2\left[\hat{\psi}_{+} \sin (X+Y)-\hat{\psi}_{-} \sin (X-Y)\right] \\
& +2 \hat{\kappa}\left[\hat{\psi}_{-} \cos (X-Y)-\hat{\psi}_{+} \cos (X+Y)\right]-U_{\mathrm{ph}}
\end{aligned}
$$

where $X \doteq k_{x}\left(x-v_{x} t\right), Y \doteq k_{y}\left(y-v_{\mathrm{ph}} t\right), U_{x} \doteq k_{x} v_{x}, U_{\mathrm{ph}} \doteq k_{y} v_{\mathrm{ph}}, \hat{\psi}_{ \pm} \doteq k_{x} k_{y} \phi_{ \pm}(1-$ $b$ ), and $\hat{\kappa} \doteq \kappa / k_{x}$. Note that $U_{\text {ph }}$ is the average of the real frequencies of the + and - modes, and $U_{x}$ is half the frequency difference.

Consider the flow given by Eqs. (27a) and (27b) for fixed $\hat{\psi}_{ \pm}$, with $\hat{\psi}_{+} \simeq \hat{\psi}_{-}$(but not necessarily equal). For typical gyrokinetic simulation runs, $\hat{\psi}_{+} \simeq \hat{\psi}_{-} \simeq 0.05$ and $U_{\mathrm{ph}} \simeq 0.05$, while $U_{x} \leqslant 0.004$, so that the frequency associated with the $x$ motion of the wave is much smaller than those associated with the $y$ motion and the $E \times B$ trapping. Thus, for times of the order of the wave period and of the " $\boldsymbol{E} \times \boldsymbol{B}$ trapping time" $\tau_{\mathrm{EB}} \doteq \pi /\left(2 \sqrt{\hat{\psi}_{+} \hat{\psi}_{-}}\right), U_{x}$ can be neglected in Eq. (27a). In this approximation, the above system of equations has a time-independent Hamiltonian,

$$
H=e^{\dot{\kappa} x}\left(\hat{\psi}-U_{\mathrm{ph}}\right)
$$

where

$$
\hat{\psi} \doteq 2\left[\hat{\psi}_{-} \cos (X-Y)-\hat{\psi}_{+} \cos (X+Y)\right]
$$

In the remainder of this paper, wherever streamlines are shown $U_{x}$ will be neglected so that the instantaneous velocity streamlines are the contours of some Hamiltonian.

Consider the case $\kappa=0$. The resulting streamlines have been studied by $\mathrm{Ching}^{14}$ in the case where $\hat{\psi}_{+}=\hat{\psi}_{-}$. In the limit $U_{\mathrm{ph}}=0$, the velocity streamlines are just the contours of $\hat{\psi}$ (or $\phi$ ). This is the limit of strong $\boldsymbol{E} \times \boldsymbol{B}$ trapping. In the opposite limit where $U_{\mathrm{ph}} \gg \hat{\psi}_{ \pm}$, the velocity streamlines are just the lines $Y=$ constant. As $\phi_{ \pm}$increases, the velocity streamlines bend as a result of the $X$ motion. Increasing $\phi_{ \pm}$further, eventually the $Y$ component of the $\boldsymbol{E} \times \boldsymbol{B}$ drift becomes equal to $U_{\mathrm{ph}}$ (occurring first at $X=0, Y=\pi / 2$ for $\hat{\psi}_{+}=\hat{\psi}_{-}=U_{\text {ph }} / 2$ ). Just above this threshold two elliptic points, a separatrix, and the associated $E \times B$-trapped trajectories appear. These qualitative properties of the gyrocenter trajectories are also present for $\kappa=0.214$, as shown in Figs. 21. These figures shows a sequence of contours of $H$ in Eq. (28a) for increasing $E \times B$ nonlinearity. (Note that in these fir $r$ - sres, the axes represent $x / L_{x}$ and $y / L_{y}$ ).

Figures 22 and 23 show the contours of $\hat{\psi}$ from Eq. (28b) and $H$ from Eq. (28a) respectively, for cases with $\hat{\psi}_{+}=\hat{\psi}_{-}$(solid contours), $\hat{\psi}_{+}<\hat{\psi}_{-}$(dotted), and 
$\hat{\psi}_{+}>\hat{\psi}_{-}$(dashed) overlayed. Note, in Fig. 22, that for $\psi_{+}>\psi_{-}$, there are unbounded contours which, when averaged over many wavelengths, follow the lines $X+Y=$ const. For $\psi_{+}<\psi_{-}$, they follow $X-Y=$ const. For $\psi_{+}=\psi_{-}$, the only unbounded contours are separatrices. As can be seen by comparing the respective contours of $\phi$ and $H$ in Figs. 22 and 23, the inequality between $\hat{\psi}_{+}$and $\hat{\psi}_{-}$affects the contours of $H$ (and hence the trajectories) far more strongly near the potential minima than near the maxima. The quantity representing this effect on the contours that is easiest to calculate analytically is the displacement in the $y$ direction of the $O$ point from its respective potential maximum or minimum. From Eqs. (27a) and (27b), the $Y$ displacement of an $O$ point of $H$ due to unequal $\hat{\psi}_{+}$and $\hat{\psi}_{-}$(for small relative inequalit $j$ between $\hat{\psi}_{+}$and $\hat{\psi}_{-}$) is given by

$$
\Delta Y=\epsilon \Delta X,
$$

where $\Delta X$ is the displacement in the $x$ direction of that $O$ point from the corresponding maximum or minirsum of $\hat{\psi}$ and $\epsilon$ is given by $E_{q}$. (26).

Now consider the shifts of the $O$ points in the $x$ direction. The $O$ point associated with the potential maximum will be denoted the $O_{+}$point and that associated with the potential minimum will be denoted the $O$ - point. The $U_{\text {ph }}$ term in $H$ of Eq. (28a) shifts the $O_{+}$point to the left and the $O_{-}$point to the right by an amount

$$
\Delta X_{ \pm}=\mp U_{\mathrm{ph}} /\left(\hat{\psi}_{+}+\hat{\psi}_{-}\right) \text {, }
$$

while the $\hat{\kappa}$ term shifts both $O$ points to the right by an amount

$$
\Delta X_{ \pm}=\hat{\kappa} .
$$

Thus, the $O_{+}$point is much closer to the potential maximum than the $O_{-}$point is to the potential minimum. When the + and - modes exchange energy, the resulting $Y$ shift of the $O_{-}$points of $H$ is thus given by

$$
\Delta Y_{ \pm}=\epsilon(t)\left[\hat{\kappa} \mp \frac{U_{\mathrm{ph}}}{\hat{\psi}_{+}+\hat{\psi}_{-}}\right] .
$$

Thus the $Y$ displacement of the $O$ points and of the surrounding bounded contours of $H$ associated with the minimum of $\phi$ is much greater than the corresponding $Y$ shifts of the $O_{+}$point and of the contours of $H$ near the potential maximum. For the purpose of making a qualitative argument, the region of good trapping (in which the gyrocenters are trapped for many $\tau_{\mathrm{EE}}$ 's) is roughly the intersection of the trapped regions of the instantaneous velocity field (given by Eqs. (27a) and (27b) over the time of the energy exchange (viewed in the wave frame). The time dependence of $\phi_{+}$ and $0_{-}$thus disrupts the $E \times B$ trapping near the potential minima far more than near the potential maxima. The inclusion of non-zero $U_{x}$ causes both the $O_{+}$point 
and the $O_{-}$point to become attracting, with the rate of contraction given by Eq. (5) as $\nabla_{\perp} \cdot v_{\text {perp }}=\hat{\kappa} U_{x}$.

Summarizing this picture of the trapping of the ion gyrocenters, the electrostatic potential contours represent a system of vortices that flip-flop with time due to the exchange between the + and - modes. This potential is strong enough for the $\boldsymbol{E} \times \boldsymbol{B}$ drift to overcome the $y$ motion of the particles in the wave frame due to the phase velocity of the waves, thus causing $E \times B$ trapping. The flip-flop of the potential contours disrupts the $E \times B$ trapping near the potential rninima more strongly than near the maxima. The $z$ motion of the waves causes particles to be attracted towards the mean $\mathrm{O}$ points of the velocity field. The combination of $\boldsymbol{E} \times \boldsymbol{B}$ trapping and the attraction causes the ion gyrocenter density to increase near the maxima of the potential. Near the minima the trapping is weaker, causing the well-trapped region there to be smaller. The enhanced density regions, being $\boldsymbol{E} \times \boldsymbol{B}$ trapped, are advected in the $x$ direction with the waves, thus resulting in an advective contribution to the ion gyrocenter flux. In the collisional $\theta=0.002$ runs of the gyrobinetic code, where the flux is high, this mechanism provides an important contribution to the ion flux. In the runs of the full gyrokinetic code globs are only observed at the potential maxima, while in the non-self-consistent code they are also seen at the minima. Self-consistent effects, in addition to the exchange between the + and - modes, must therefore be acting to suppress the formation of the globs at the potential minima. These effects will be further discussed in Sec. IX.

\section{B. Electrons}

The collisionless electron guiding center motion is more complicated than the ion motion because of the importance of the parallel motion and pitch-angle scattering. The parallel motion is important in the linear regime in that it provides the inverse Landau damping causing the linear instability that drives the modes; it will be seen to be important in the nonlinearly saturated states as well. In the $2 \frac{1}{2}$-dimensional geometry, the existence of the Hamiltonian given in Sec. IV allows some major simplifications to be made.

From Eq. (15), the instantaneous streamlines are given by a two-dimensional system with Hamiltonian

$$
H(x, y)=e^{\kappa x}\left\{\dot{\phi}+\frac{\theta v_{\mid \rho^{0}}-v_{\mathrm{ph}}}{\kappa}+\frac{m_{\mathrm{i}}}{m_{e}} \frac{\theta^{2}}{\kappa^{2}}\left[\kappa\left(x_{0}-x\right)+1\right]\right\},
$$

where $x_{0}$ and $v_{\| 0}$ are, respectively, the initial $x$ coordinate and parallel velocity of a particle. The terms resulting from the parallel acceleration have been collected towards the right. The existence of this Hamiltonian implies that (when $v_{x}=0$ ) the streamlines cannot become chaotic and have no sources or sinks, despite the presence of both parallel and $\boldsymbol{E} \times \boldsymbol{B}$ trapping. 
The two values of $\theta$ used in the runs described in Sec. III are associated with different orderings between the $\boldsymbol{E} \times \boldsymbol{B}$-and parallel trapping times. The characteristic $E \times B$-trapping time can be defined as $\tau_{E B} \doteq \pi /\left(2 k_{x} k_{y} \phi_{0}\right)$ and the parallel trapping time as $\tau_{\|} \doteq\left(\pi / k_{y} \theta\right) \sqrt{\left(m_{e} / m_{\mathrm{i}} \phi_{0}\right)}$, where $\phi_{0}=\bar{\phi}_{+}=\bar{\phi}_{-}$is the time average of the + and - mode amplitudes. For $\theta=0.002, \tau_{\mathrm{EB}} / \tau_{\|} \simeq 0.2$, while for $\theta=0.01$, the swo time scales are comparable. It is straigtforward to show ${ }^{19}$ that when $\tau_{\mathrm{EB}} / \tau_{\mid} \ll 1$, the only effect of the parallel acceleration discernible for displacements of the order of a wavelength is a small $\left(O\left(\left[\tau_{E B} / \eta_{1}\right]^{2}\right)\right)$ shift in the $O$ point position and in the $E \times B$-trapping frequency. An analogous statement holds for $\tau_{\|} / \tau_{E B} \ll 1$. Thus, in the two limits of the ratios of these two time scales, perturbation theory can be used without the need to worry about sudden changes in the topology (over length scales of the order of a few wavelengths). For $\theta=0.002$, the effect of the parallel acceleration can, therefore, be treated as a slow change of $v_{\| 0}$ in the Hamiltonian of Eq. (31) without the parallel acceleration terms,

$$
H_{0}=\mathrm{e}^{\kappa x}\left\{\phi+\frac{\theta v_{\| 0}-v_{\mathrm{ph}}}{\kappa}\right\} \text {. }
$$

For fixed $v_{\| 0}$, this Hamiltonian is formally the same as the ion Hamiltonian of Eq. (16) that was also used in the previous subsection. The streamlines for the electron guiding-center flow induced by the potential of $\mathrm{E}_{\mathrm{q}}$ (19a), iscluding parallel acceleration, are almost indistinguishable from those shown in Fig. 23 which does not contain parallel acceleration.

\section{1. $\vec{E} \times \vec{B}$ Detrapping due to Parallel Acceleration}

For $\theta=0.01$ runs, the parallel acceleration does affect the streamlines significantly over one wavelength. Figure 24 shows the contours of $H$ in Eq. (31) for the same three values of $\epsilon$ as in Fig. 23, with $\phi_{+} \simeq \phi_{-} \simeq 0.04$ and $\theta=0.01$. All contours are for a single value of $C_{1}\left[\doteq v_{i 0}+m_{\mathrm{j}} \theta_{x_{0}} / m_{\mathrm{e}}\right.$ from Eq. (13)]. This figure shows that, for $\theta=0.01$, if an initially trapped electron is moved in the $x$ direction by $\boldsymbol{E} \times \boldsymbol{B}$ diffusion, then the parallel acceleration can cause it to become untrapped for an $x$ displacement of less than a wavelength.

This same detrapping mecharism can occur in the $\theta=0.002$ case, the main difference being that the electron has to undergo a much larger $x$ displacement before becoming untrapped. The $I$ displacement that an electron or ion must undergo before becoming $E \times B$-detrapped can easily be estimated by comparing the parallel acceleration term and the $E \times B$ drift term in the equation for $\dot{Y}$ resulting from Eq. (11b) when the potential of Eq. (19a) with $\phi_{+}=\phi_{-}=\phi_{0}=$ const is inserted. This equation can be written, for a particular choice of origin, as

$$
\dot{Y}=4 k_{x} k_{y} \phi_{0} \cos X \sin Y+\zeta \theta\left(X_{0}-X\right)
$$


where $\zeta$ is given by Eq. (12) (zero shear case). The $x$ displacement $\Delta X$ for complete detrapping to occur is thus given by

$$
\zeta \theta \Delta X=4 k_{x} k_{y} \phi_{0} .
$$

Upon taking, for example, the saturation level $\phi_{0} \sim 0.07$, characteristic of the $\nu_{\mathrm{ei}}=0.01$ runs for both values of $\theta$ used, we obtain

$$
\frac{\Delta X}{2 \pi} \sim \begin{cases}4 & \text { (electrons, } \theta=0.002), \\ 0.2 & \text { (electrons, } \theta=0.01) \\ 8 \times 10^{3} & \text { (ions, } \theta=0.002) \\ 3 \times 10^{2} & \text { (ions, } \theta=0.01) .\end{cases}
$$

From these numbers and from the value of the $E \times B$ drift, it is easily seen that during the entire time of a typical simulation run, the ion parallel acceleration is quite ineffective in modifying the ion $E \times B$ motion. Figure 25 shows three sets of streamlines for $\theta=0.002$, with $\bar{C}_{1} \doteq\left(k_{x} / 2 \pi\right)\left[m_{\mathrm{e}} /\left(m_{\mathrm{i}} \theta\right)\right] C_{1}=\left(k_{x} / 2 \pi\right)[x+$ $\left.\left(v_{\|} m_{e} / m_{\mathrm{i}} \theta\right)\right]=0$ (solid), 2 (dotted), and 4 (dashed). This figure shows the presence of bounded trajectories for $\bar{C}_{1}=0$ and 2 but not for $\bar{C}_{1}=4$. Figure 26 is a similar figure for the case $\theta=0.01$, showing contours for $\bar{C}_{1}=0$ (solid), -0.2 (dashed), and 0.2 (dotted). This figure shows graphically that a smaller $x$ displacement is required for the parallel acceleration to cause $E \times B$ detrapping for $\theta=0.01$ than for $\theta=0.002$.

\section{Effects Limiting Electron Glob Formation}

The electron globs observed in the $\theta=0.002$ cases constitute density perturbations somewhat larger than the adiabatic response. The mechanism by which they form is basically the same as for the ion globs. With the insight of the previous subsections in hand, we are in a position to consider the differences between the elec $^{+}$:on and ion gyrocenter motions on the glob formation process. For the electrons in the $\theta=0.002$ cases, the compression factor allowed by the displacement given by Eq. (33) is $\exp (\kappa \Delta x)=\exp (\hat{\kappa} \Delta X) \sim 10^{3}$. There are three other effects, however, which reduce the maximum possibie compression factor. These are as follows.

- The $x$ motion of the globs is associated (through the constancy of $C_{1}$ ) with secular parallel acceleration of the initially very-well-trapped electrons that produce the observed globs. The resultant slow change in $v_{\| 0}$ in Eq. (32) causes a shift in the $O$ points given by Eq. (29). This shift in turn results in an increase in $\Delta Y$ as given by Eq. (30). Empirically from the non-selfconsistent ion simulations, this detrapping occurs once the parallel acceleration has shifted the $O$ point by $\Delta X_{0} \simeq 0 . \bar{J}$ for the parameter values 
used. From Eq. (13), it is straightforward to show that a given displacement, $\Delta X_{O}$, of the $O$ point can be caused by a change in the parallel velocity $\Delta v_{\|}=\Delta X_{O} \times\left(4 k_{x} k_{y} \phi_{0}\right) /\left(k_{y} \theta\right) \sim 1.3 v_{\text {le }}$, which can be caused by the parallel acceleration associated with an $x$ motion (for example, of the whole system of waves), $\Delta X=\left(m_{\mathrm{i}} \theta / m_{\mathrm{e}}\right) \Delta v_{\|} \sim 16$, where the numerical values are for the runs with $\theta=0.002$ and $L_{x}=L_{t}$. The maximum compression factor is thus $\exp (\hat{\kappa} \Delta X) \sim 60$ for these runs.

- The spread in parallel velocity of the electrons reduces the relative strength of the globs directly since, from the arguments given in the previous point, only those electrons with parallel velocities roughly in the interval $-1.3 v_{\text {te }}<$ $v_{\|}<1.3 v_{\imath e}$ are well enough $\boldsymbol{E} \times \boldsymbol{B}$-trapped to be able to contribute to the density increase in the globs. For a Maxwellian distribution the fraction of such electrons is roughly $75 \%$.

- Collisions can scatter an electron gyrocenter out of this velocity interval in roughly a collision time. For $\nu_{e j}=0.01$, the highest collision frequency used, and $k_{x} v_{x} \sim 0.004$ the density increase due to the compression over a collision time is roughly 0.25 . This is somewhat larger than the adiabatic response and comparable to the actual electron density increases seen both in the full and the non-self-consistent simulations.

\section{Exact Non-Self-Consistent Solutions}

There are exact solutions for the electron distribution in a given potential field related to those observed in the simulations that shed considerable light on the electron behavior. For $\phi_{+}=\phi_{-}=$const. and $v_{x}=0$, there is a class of such exact solutions, in the limiting cases of $\nu_{\mathrm{ei}}=0$ and $\kappa=0$, which are time-independent in the wave frame and are easily obtained. Also, when both $\nu_{\text {ei }}$ and $\kappa$ are nonzero, a useful bound on the time integral of the electron gyrocenter flux can still be obtained. This bound is much lower than the value measured in the collisional $\theta=0.002$ runs. Therefore, either a time-dependent energy exchange between the + and - modes or a nonzero $v_{x}$ is necessary for consistency with the observed values of the gyrocenter flux.

The travelling wave solution for $\kappa=0$ is a simple generalization of the standard adiabatic response. The gyrokinetic Vlasov equation for the electrons, for solutions that are time-independent in the wave frame, can be written as

$$
\frac{\partial}{\partial x}(\dot{x} f)+\frac{\partial}{\partial y}(\dot{y} f)+\frac{\partial}{\partial v_{\|}}\left(\dot{v}_{\|} f\right)=C(f)
$$

where $\dot{x}, \dot{y}$, and $\dot{v}_{\|}$are given by Eqs. (11a)-(11c), with $\kappa=0$. This system has the 
exact solution $f=F_{\mathrm{M}}^{\mathrm{e}}\left(v_{\|}\right) n(\boldsymbol{x})$, where

$$
n(\boldsymbol{x})=\exp \left(H_{i}\right)
$$

and where $H_{i}=\phi-v_{\mathrm{ph}} x$ is the first Hamiltonian of Eq. (17), valid for zero parallel velocity and $x$. Note that there is a density gradient in this "equilibrium" solution associated with the $v_{\mathrm{ph}} x$ term in $H_{i}$. This density gradient is the nonlinear manifestation of the $\omega$ term in the $\omega .-\omega$ factor in the phase shift $\alpha_{\text {lin }}$ of Eq. (7). Thus, for example, waves with a real frequency $\omega>\omega_{\text {. }}$ can drive a particle flux in the direction of the density gradient.

For $\nu_{\mathrm{c} i}=0$ [so that $C(f)=0$ ], but $\kappa \neq 0$, Eq. (34) can be solved by the method of characteristics to yield

$$
f=\exp (\kappa x) \hat{f}\left(H, C_{1}\right)
$$

where $\hat{f}$ is an arbitrary function of $H$ and $C_{1}$ which from Eqs. (12), (13), and (15), are given by $C_{1}=v_{\|}+\left(m_{\mathrm{i}} / m_{\mathrm{e}}\right) \theta x$, and

$$
H=\mathrm{e}^{\kappa x}\left\{\phi-\frac{m_{\mathrm{i}}}{m_{\mathrm{e}}} \frac{\theta^{2}}{\kappa} x+\frac{1}{\kappa^{2}}\left[\left(\theta C_{1}-v_{\mathrm{ph}}\right) \kappa+\frac{m_{\mathrm{i}}}{m_{\mathrm{e}}} \theta^{2}\right]\right\} .
$$

When both $\kappa \neq 0$ and $\nu_{\mathrm{e}} \neq 0$, there are no such simplifications that yield simple analytical travelling wave solutions. However, an estimate of the maximum available time-integrated fux for the case of $\phi_{+}=\phi_{-}=\phi_{0}$ can be obtained as follows. For a suitable choice of origin, Eq. (27a) (which is equally valid for electrons) becomes

$$
\dot{X}=-4 k_{x} k_{y} \phi_{0} \sin X \cos Y \text {. }
$$

Thus $\dot{X}=0$ for $X=n \pi$, for any integer $n$, so that these lines form boundaries through which electron guiding center orbits cannot pass (with or without collisions). As can be seen from the solution of $\mathrm{Eq}$. (36), the main effect of the $\kappa$ term in the equation of motion is to cause the equilibria to be $\exp (\kappa x) \times$ (some function of the constants of motion). Density profiles that vary piecewise as $\exp (\kappa x)$ in this ("multiscale") model represent piecewise-level density profiles in the true physical system. The constraint resulting from Eq. (37) allows the relaxation to occur only between the lines $x=\pi \pi / k_{x}$, resulting in profiles roughly as shown in Fig. 27. Since for the parameters of the simulation $\pi \kappa / k_{x} \sim 0.8$ while elo(x)|/Te $\leqslant 0.1$, the adiabatic response to the potential due to the parallel motion is small cornpared with this relaxation to "flattened" density profiles, and can be neglected for the purpose of making this estimate. To obtain the bound on the time integral of the tlux, the one-dimensional continuity equation

$$
\frac{\partial n}{\partial t}+\frac{\partial \Gamma_{e}}{\partial x}=0
$$


is multiplied by $x$ and integrated over time and over a half period bounded by the $\dot{x}=0$ lines in the $x$ direction, yielding

$$
\int_{t_{1}}^{t_{2}}\left\langle\Gamma_{e}\right\rangle d t=\int_{A}\left\langle\left\langle n\left(t_{2}\right)-n\left(t_{1}\right) \mid x\right\rangle d A\right.
$$

where the angle brackets denote a spatial average. For the estimate of the timeintegrated flux, a piecewise linear approximation to the relaxed density profile (also shown in Fig. 27) is adequate. Upon taking $n\left(t_{1}\right)$ to be the initial fiat density profile and $n\left(t_{2}\right)$ to be the relaxed profile, one obtains

$$
\int\left\langle\Gamma_{e}\right\rangle d t=\frac{1}{12} \kappa\left(\frac{\pi}{k_{x}}\right)^{2} \simeq 0.3,
$$

(in units of $n_{0} \rho_{\mathrm{s}}$ ) for the values of $\kappa$ and $k_{x}$ used here. This value is in good agreement with the results of the non-self-consistent gyrocenter code for zero energy exchange given in Sec. VI. For the $\theta=0.002, \nu_{e}=0.001$ and 0.01 runs of the full gyrokinetic code, the time-integrated flux is of order $1-10$. Thus, the time dependence of the electrostatic potential is necessary to account for the electron flux in these cases.

\section{VIII. $\vec{E} \times \vec{B}$ DIFFUSION IN SEMI-COHERENT WAVE FIELDS}

\section{A. Analytical Estimates}

In this section, we give simple analytical estimates of the $\boldsymbol{E} \times \boldsymbol{B}$ diffusion modified by coherent effects, parallel acceleration and collisions as was found Sec. VI. These analytical estimates are in good agreement with the results from the nonself-consistent simulations.

The diffusive particle flux for either species can be written in the form

$$
\Gamma=\kappa_{\mathrm{eff}} D_{\mathrm{ql}} \xi_{\mathrm{r}} \xi_{\mathrm{eoh}} \xi_{\mathrm{ac}},
$$

where $\kappa_{\text {eff }}$ is the effective logarithmic density gradient (corrected for the phase velocity of the waves), $D_{\mathrm{ql}}$ is the "quasilinear" $\boldsymbol{E} \times \boldsymbol{B}$ diffusion coefficient, and the $\xi$ 's are multiplicative factors less than 1 which represent the effects of incomplete $\boldsymbol{E} \times \boldsymbol{B}$ trapping $\left(\xi_{r}\right)$, wave coherence $\left(\xi_{\mathrm{cot}}\right)$, and parallel acceleration, and collisions $\left(\xi_{a c}\right)$. Each of these will be explained in turn.

The effective logarithmic density gradient $\kappa_{\text {eff }}$ is the difference between the actual logarithmic density gradient and that to which drift waves with a real frequency $w$ drive the density profile. Its value, as found in the discussion immediately following Eq. $(35)$ is

$$
\kappa_{\mathrm{eff}}=\kappa\left(1-\frac{\omega}{\omega_{s}}\right) .
$$


The quasilinear diffusion coefficient $D_{\mathrm{ql}}$ is the diffusion coefficient valid for completely chaotic trajectories. It can be estimated via a random walk argument.

$$
D_{\mathrm{qi}} \simeq(\Delta x)^{2} / 2 \tau,
$$

where $\Delta x$ is the step size and $\tau$ is the detrapping time. The detrapping time is given by the time scale of variation of the potential due to the energy exchange, which empirically is found to be comparable to the $E \times B$-trapping time $\tau_{E B}=$ $2 \pi /\left(4 k_{x} k_{y} \phi_{0}\right)$, where $\phi_{0}$ is a typical value of the amplitude of the + or - mode. During the detrapping time a typical gyrocenter undergoes a displacement $\Delta x$ given by $k_{x} \Delta x \sim \pi$. Thus, the diffusion coefficient is given by

$$
D_{\mathrm{ql}} \simeq \pi \frac{k_{y}}{k_{x}} \phi_{0} \simeq 0.2,
$$

(in units of $\rho_{3} c_{3}$ ) where the typical simulation values $k_{y} / k_{z}=1$ and $\phi_{0}=0.07$ have been used. The non-self-consistent gyrocenter simulation results, with all parameters other than $v_{x}$ (which is set to zero) fitted to the gyrokinetic code run with $\theta=0.002$ and $\nu_{\mathrm{ej}}=0.001$, give $D \lessgtr 0.033$, indicating that coherent effects are important for both particle species.

Since the potential in this non-self-consistent simulation consists entirely of the + and - modes (and their complex conjugates), the particle flux is associated with a phase shift between the + and - modes of the gyrocenter density and those of the potential. This phase shift can represent either $\boldsymbol{E} \times \boldsymbol{B}$ diffusion or the advection of globs.

The factor $\xi_{\mathrm{r}}$ is a correction representing the fraction of gyrocenters in resonance as will now be explained. The resonant gyrocenters can diffuse most rapidly since most of the trapping and detrapping occurs at the X points of the orbits in the time-averaged potential. The streamlines for the resonant gyrocenters have a grid of $X$ points as shown in Fig. 28. After undergoing $E \times B$ detrapping and retrapping at one $X$ point, a resonant gyrocenter is rapidly carried past the next $X$ points to the right, where it can be $\boldsymbol{E} \times \boldsymbol{B}$-detrapped and retrapped into the next cell. A nonresonant gyrocenter, on the other hand, must pass through a band of passing trajectories before it can reach the next $X$ points. The diffusion through the band of passing trajectories is much slower than the nondiffusive type of $E \times B$ flow between $X$ points that a resonant gyrocenter undergoes. To estimate $\xi_{r}$, we assume a sharp cutoff of the diffusion as a function of parallel velocity. Neglecting the $\kappa$ term in the consideration of the geometry of the trapped and passing regions, the passing trajectories that are least perturbed by the energy exchange between the two modes are the most strongly passing trajectories. In terms of the variables $X=k_{x} x, Y=k_{y} y$, the gyrocenter Hamiltonian of Eq. (32), without the $\kappa$ term or the parallel acceleration for the potential of Eq. $(19 \mathrm{a})$ with unequal $\phi_{+}$and $\phi_{-}$, is

$$
H=\omega_{E B}(\sin X \sin Y+\epsilon \cos X \cos Y)-\omega_{D o p} X,
$$


where $\omega_{\text {Dop }} \doteq \omega_{T}-k_{\|} v_{\|}$is the Doppler-shifted real frequency. An example of a least-trapped trajectory, shown in Fig. 29, is the trajectury passing through $(X, Y)=(-\pi / 2,0)$. In situation $A$, the energy exchange is too weak to significantly perturb this trajectory, while in situation $B$ the energy exchange has caused an $X$ displacement of $-\pi$ when going from $Y=\pi(X=3 \pi / 2)$ to $Y=0(X=\pi / 2)$. The transition between the two situations is a quite sudden one and is easily obtained as follows. At $(X, Y)=(\pi / 2,0)$, we have $H=-\omega_{\text {Dop }} \pi / 2$, so that the $X$ value of this trajectory at $Y=\pi$ is given by

$$
H=-\omega_{\mathrm{Dop}} \pi / 2=-\omega_{E B} \epsilon \cos X-\omega_{\mathrm{Dop}} X
$$

or

$$
\cos X+\beta(X-\pi / 2)=0,
$$

where $\beta \doteq \omega_{\text {Dop }} /\left(\epsilon \omega_{E B}\right)$. For $\beta<1$, there are two solutions on the interval $[0,2 \pi)$, corresponding to situation $B$, while for $\beta>1$ there is only one solution, corresponding to situation $A$. We call situation B "resonant" and situation A "nonresonant." Thus, the condition for the electrons or the ions to be resonant is

$$
\omega_{\mathbf{r}}-k_{\|} v_{\|} \lesssim \omega_{E B}
$$

For the $\theta=0.002, L_{x}=L_{y}$ gyrokinetic code runs, the typical value of the energy exchange is $\epsilon \simeq 0.2$, so the parallel velocity interval (both sides of the resonance) is $\Delta v_{\|} / v_{\mathrm{te}} \simeq 1( \pm 0.1)$. The reduction factor $\xi_{\mathrm{r}}$ between the cases with a thermal distribution of electrons (without parallel acceleration) and with all resonant electrons, is thus

$$
\xi_{\mathrm{r}} \simeq \frac{1}{\sqrt{2 \pi}} \exp \left\{-\frac{1}{2}\left(\frac{\omega_{\mathrm{r}}}{k_{\|,} v_{\mathrm{te}}}\right)^{2}\right\} \simeq \frac{2 \epsilon \omega_{E B}}{k_{\|}} F_{\mathrm{M}}^{\mathrm{e}}\left(v_{\text {res }}\right) \simeq 0.34,
$$

where the second expression is valid for $\epsilon \omega_{E B} \ll k_{\|} v_{\text {le }}, v_{\text {res }} \doteq\left(\omega_{\mathrm{r}} / k_{\|} v_{\text {te }}\right)$, and the numerical value is for $\omega_{\mathrm{r}}=0.042$ (which is typical for the $\theta=0.002$ simulations). This is in good agreement with the ratio of the gyrocenter fluxes between the nonself-consistent code runs where there is a Maxwellian velocity distribution but no parallel acceleration and where all particles are exactly resonant from the non-selfconsistent gyrocenter code results.

The factor $\xi_{\text {coh }}$ takes into acco" ${ }^{*}$ the following two effects of the spatial coherence of the wave fields. Even for the resonant electrons. the $E \times B$ detrapping and retrapping are effective only for guiding centers which come sufficiently close to the $X$ points. Also, proximity to the bounding trajectories implies a nonlinedrly modifled $\boldsymbol{E} \times \boldsymbol{B}$-trapping frequency that is lower than that for deeply $\boldsymbol{E} \times \boldsymbol{B}$-trapped trajectories. The consequences for the diffusion of these two effects will now be 
estimated. For the estimate of the fraction $f_{\text {der }}$ of resonant gyrocenters that are affected by the motion of the $\mathrm{X}$ points, assume the density to be uniform. Then $f_{\text {atr }}$ is just the fraction of the $x-y$ plane occupied by passing trajectories for the maximum value of the inequality between $\phi_{+}$and $\phi_{-}$. For small $\epsilon$, as defined in Eq. (42) [and originally in Eq. (26)], this shaded area is easily evaluated by subdividing the passing region as shown in Fig. 30, and using a small $\epsilon$ perturbative result for the equation for the boundary of region $A$. The result is

$$
f_{\mathrm{der}} \simeq \frac{8 \epsilon \ln \left(\epsilon^{-1}\right)}{\pi^{2}} \simeq 0.3
$$

for $\epsilon \simeq 0.2$. The time taken for a gyrocenter on the most strongly passing trajectory to move from $Y=0$ to $Y=\pi$ can be estimated for small $\epsilon$ by using a similar kind of subdivision as used for the estimate of $f_{\text {der }}$. The result is

$$
\tau_{\text {t⿱ }} \simeq \frac{\ln \left(4 e^{-1}\right)}{2 k_{x} k_{3}\left|\phi_{ \pm}\right|} \simeq \tau_{E B}
$$

so that

$$
\xi_{\mathrm{coh}} \simeq f_{\mathrm{dtr}} \tau_{\pi} \simeq 0.3
$$

for the parameters used here. The motion on the less strongly passing trajectories is even slower, so that a gyrocenter on one of these can no longer make the $X$ displacement of $\pi$ in an attocorrelation time, resulting in the further decrease in the diffusion coefficient.

Finally, the factor $\xi_{\mathrm{ac}}$ represents the balance between the reduction in the diffusion coefficient due to the depletion of the resonant population by the parallel acceleration and the maintainance of that resonant population by collisions. Let $\Delta v\left(\simeq v_{\mathrm{te}}\right)$ from Eq. (43) be the velocity interval for which the diffusion is effective, $F$ the value of the distribution function with no depletion, and $f$ the actual value. Assume that only the electrons in the resonant interval diffuse and are accelerated. The reduction factor due to the depletion is thus $f / F$. The rate of depletion of $f$ by the parallel acceleration is given by

$$
\dot{f}_{\text {acc }} \simeq-\frac{\left\langle\dot{v}_{11}\right\rangle}{\Delta v} f \simeq-\frac{m_{i} \theta \Gamma_{\text {rea }}}{m_{\mathrm{e}} n_{\text {res }}} \frac{f}{\Delta v},
$$

where $\Gamma_{\text {res }}$ and $n_{\text {res }}$ are, respectively, the resonant electron flux and number density, and $\left(i_{i 1}\right)$ is the mean parallel acceleration of a resonant electron due to its diffusion. Equation (13) was used in obtaining the final expression. The rate at which the collisions replenish $f$ in the resonant region follows approximately from the diffusive term in the Lenard-Bernstein collision operatior $\dot{f}=\nu_{\mathrm{ei}} \partial_{v}^{2} f$. We (crudely) take the gradient of the distribution function in the resonant interval to be $-(2 / \Delta v)^{2}(F-f)$, 
according to Fig. 31. From the diffusive part of the collision operator of Eq. (3) the rate at which the parallel velocity diffusion due to the collisions replenishes $f$ in the resonant region is

$$
f_{\text {coll }} \simeq \nu_{\text {ei }}\left(\frac{2 v_{\text {te }}}{\Delta v}\right)^{2}\left(1-\frac{f}{F}\right) F \text {. }
$$

Upon balancing these and assuming $f / F \simeq 1$, the reduction factor becomes

$$
\zeta_{\text {ace }}=\frac{f}{F} \simeq 1-\left(\frac{m_{\mathrm{i}} \theta}{4 m_{\mathrm{e}} v_{\mathrm{te}}} \frac{\Gamma_{\mathrm{rea}}}{n_{\mathrm{res}}} \frac{1}{\nu_{\mathrm{ej}}}\right) \simeq \begin{cases}0.85 & \left(\nu_{\mathrm{ei}}=0.001\right), \\ 0.99 & \left(\nu_{\mathrm{ei}}=0.01\right) .\end{cases}
$$

The value $\Gamma_{\text {res }} / n_{\text {res }} \simeq 0.007$ from the non-self-consistent electron gyrocenter simulation with all of the electrons at resonance has been used. This crude estimate somewhat underestimates the depletion. The reason for this is that, as Fig. 30 shows, the electrons which carry most of the flux are in a fraction $f_{d t r}$ of the configuration space. If the mixing between the areas which produce the diffusion and those which do not is weak, then those electrons which are transported have a somewhat higher value of $\Gamma_{\text {res }} / n_{\text {res }} \simeq 0.007 / f_{\text {dtr. }}$ This gives

$$
\zeta_{\text {acc }} \simeq \begin{cases}0.55 & \left(\nu_{\mathrm{ei}}=0.001\right), \\ 0.95 & \left(\nu_{\mathrm{ej}}=0.0 \mathrm{I}\right),\end{cases}
$$

which is in good agreement with the ratio between the fluxes in the $v_{\mathrm{ei}}=0.001$ and 0.01 cases. This estimate cannot, however, be regarded as reliable because of the crudeness of the approxirnations to the Folker-Planck equation and the uncertainties regarding the mixing in configuration space. The non-self-consistent code runs of Sec. VI constitute a more reliable solution of the Fokker-Planck equation and give

$$
\zeta_{\text {ace }} \simeq \begin{cases}0.6 & \left(\nu_{\mathrm{ei}}=0.001\right) \\ 1 & \left(\nu_{\mathrm{ei}}=0.01\right)\end{cases}
$$

to within measurement error.

Combining these factors gives a result for the diffusion coefficient

$$
\Gamma= \begin{cases}0.003 & \nu_{\mathrm{ei}}=0.01 \\ 0.0018 & \nu_{\mathrm{ei}}=0.001\end{cases}
$$

which is in good agreement with the full particle simulation result.

\section{INTERPRETATION OF PHENOMENA AND THEIR RELEVANCE}

\section{A. Validity of the " $i \delta_{\mathrm{e}}$ " Model}

It was mentioned in Sec. I, that the linear electron phase shift has been used in several studies of the nonlinear behavior of the collisionless drift and related 
instabilities. ${ }^{9-12}$ In this section, we show that the use of such models has a (at least approximate) nonlinear justification based on the $E \times B$ diffusion of near-resonant electrons, provided that the resonant electron population is unaltered by the drift waves (no reson:nce depopulation) and that the fluctuations are turbulent (so inat $\epsilon \geq 0.5)$.

Under the stated assumptions, we have $\zeta_{\text {coh }} \simeq \zeta_{\text {ac }} \simeq 1$, so that the electron flux is given approximately by

$$
\Gamma_{e} \simeq \kappa_{\mathrm{eff}} D_{\mathrm{ql}} \xi_{\mathrm{r}}
$$

where $\kappa_{\text {eff }}$ is the effective logarithmic density gradient given by Eq. (40). Lising the results of the previous section for $\kappa_{\mathrm{eff}}, D_{\mathrm{ql}}$, and $\zeta_{\mathrm{r}}$, it follows that

$$
\Gamma_{e} \simeq 4 k_{y}\left[\frac{\pi\left(\omega_{-}-\omega\right)}{k_{\|}} F_{M}^{e}\left(v_{\mathrm{res}}\right)\right] \phi_{0}^{2}
$$

Upon comparing this result with $\Gamma_{e}=2 k_{y} \alpha_{e}\left(\left|\phi_{+}\right|^{2}+\left|\phi_{-}\right|^{2}\right)$, derived analogously to Eq. (24a), we obtain

$$
\alpha_{e} \simeq \frac{2 \epsilon \pi\left(\omega_{.}-\omega\right)}{k_{\|}} F_{M}^{e}\left(v_{\text {res }}\right) \simeq 2 \epsilon \alpha_{\mathrm{lin}},
$$

where $\alpha_{\text {bin }}$ is the linear phase shit. The case $\epsilon=1$ corresponds to straight diagonal potential contours, while the $\epsilon=0.2$ corresponds to the semi-coherent states seen in the gyrokinetic simulations. The case of fully developed turbulence lies somewhere in between. It is, therefore, not unreasonable to take $2 \epsilon \simeq 1$, which gives the linear result. Thus, provided that the advecting fields are sufficiently turbulent and threedimensional effects (or collisions) are strong enough to prevent the depletion of the resonant electron population, the use of the $i \delta_{\mathrm{e}}$ models is justified for the study of the effect of the ion nonlinearities on drift waves.

\section{B. Velocity of the Waves in the $x$ Direction}

In this section, we explain the origin of the motion of the waves in the $x$ direction observed in the collisional $\theta=0.002$ runs.

As shown in the previous sections, given a level of the + and - modes of the electrostatic potential and a non-zero time-dependent energy exchange between these modes, there is a resultant electron flux due to the $\boldsymbol{E} \times \boldsymbol{B}$ diffusion of the electrons. The time-averaged electron response can then be written approximately as $n_{ \pm}^{e} \simeq\left(1 \mp i \alpha_{e}\right) \dot{\phi}_{ \pm}$, where $\alpha_{e}$ is the (small) phase shift between the electron density and the potential which represents the effect of the $E \times B$ diffusion. Inserting this into the approximate gyrokinetic Poisson equation Eq. (9), it is found that the ions must have a phase shift $\alpha_{i}=\alpha_{e} /\left(1+k_{1}^{2}\right.$ ) (and this is dynamically enforced through the Poisson equation via motion of the potential contours). This result 
can be represented by the diagram in Fig. 32. In the collisional small- $\theta$ cases, a substantial fraction of the ion flux is due to the advection of localized high density regions located near the potential maxima. In the limit where all of the flux is due to these globs, $\alpha_{\mathrm{i}}$ has a clear interpretation as the mean separation in the $y$ direction between the globs and their respective potential maxima. Thus, taking $\dot{X}=0$ and expanding about the 0 points in Eq. (27a), the value of this phase shift is

$$
\alpha_{i} \simeq k_{y} \Delta y=\frac{v_{x}}{4 k_{y} \phi_{0}}
$$

Note that this formula has no explicit dependence $\kappa$, although $\Delta y$ depends on $\alpha_{e}$ which in turn depends on $\kappa$. Thus we have

$$
v_{x}=4 k_{x} k_{y} \phi_{0} \alpha_{e} /\left(1+k_{\perp}^{2}\right)
$$

The actual value may depart from this somewhat through the presence of the diffusive ion flux and the fact that the actual globs are not completely concentrated. For the typical $\theta=0.002$ simulation values of $k_{x} v_{x} \$ 0.004$ and $\phi_{0} \simeq 0.06$, the $y$ displacement of the globs from the potential maxima satisfies $k_{y} \Delta y \lessgtr 0.02$. Thus, these displacements are too small to be visually discerned by looking at the $\phi$ contours and the scatter plots from the gyrokinetic simulations.

To see how this $I$-directed velocity arises dynamically, suppose that the ions have $\delta$-function density distributions at the density maxima shown in Fig. 32 . The $\boldsymbol{E} \times \boldsymbol{B}$ velocity of these points is clearly non-zero and in the $x$ direction. A small shift of the ions in the $x$ direction results, through Eq. (9), in a larger shift of the $\dot{T}$ and - modes of the potential. If, as the non-self-consistent simulations indicate, the adiabatic response and $\boldsymbol{E} \times \boldsymbol{B}$ diffusing parts of the electron distribution can move with the potential contours, then the whole wave system can move in the I direction, driven by the $\boldsymbol{E} \times \boldsymbol{B}$ advection of the ion globs. If the potential contours were unable to move, then the ion globs would be advected around the potential maxima and there would be no time-averaged ion gyrocenter flux.

\section{Estimates of the Saturation Levels}

The different dependences on the saturation level of the electron and ion fluxes enable the system to strike the balance of fluxes that yields a finite saturation level. The diffusive electron flux increases quite steadily as a function of the saturation level, because of the steady increase in the $\boldsymbol{E} \times \boldsymbol{B}$ velocity and in the number of electrons in resonance. The nonlinear contribution to the ion guiding center fiux, on the other hand, is small until the potential reaches a large enough value either for the resonance region (as defined in Sec. VIII) to extend to zero parallel velocity. which is where essentially all of the ions sit, or for glob formation to be able to occur. 
The nonlinear contribution to the ion flux increases quite sharply when either of these boundaries is crossed.

The criteria for resonant diffusion and for glob formation will now be given and compared to show that the glob formation will usually occur at lower saturation levels than the resonant ion diffusion for the parameter values used in the simulations. From Eq. (43), the criterion for resonant diffusion to occur is

$$
\frac{\omega_{\mathrm{r}}}{\omega_{E B}} \lesssim \epsilon
$$

where $\omega_{\mathrm{r}}$ is the actual (measured) real frequency and $\omega_{E B}=4(1-b) k_{x} k_{y} \phi_{0}$ is the $\boldsymbol{E} \times \boldsymbol{B}$ trapping frequency for the ions. From the non-self-consistent ion gyrocenter simulation results, it is found that globs can form if the displacement $\Delta x$ of the $O$ point of the velocity field from the potential maximum satisfies $k_{x} \Delta x \leqslant 0.5$. Using the results of Sec. VII for the shifts in the O points (associated with the potential maxima) calculated from Eqs. (27a) and (27b), this criterion becomes

$$
\left|\frac{\omega_{r}}{\omega_{E B}}-\frac{\kappa}{k_{x}}\right| \lesssim g(\epsilon) \simeq 0.5 .
$$

Here $g$ is a decreasing function of $\epsilon$, with the approximate empirical value from the non-self-consistent ion gyrocenter simulations of 0.5 for the values of $\epsilon$ typical of the $\theta=0.002$ simulations. The first term is the $X$ displacement of the $O$ point due to the phase velocity of the wave and the second tern is the displacement due to the $\kappa$ term in Eq. (27b). As the saturation level increases, so will the convective flux, rising quite sharply until the left-hand side of the above expression is zero, when the velocity $O$ point lies at the potential maximum. At this point the ion $E \times B$ trapping at the potential maxima is very strong and a further increase in the saturation level will not enhance it much. Because of the rapid increase in the non-self-consistent flux as a function of the saturation level, the balance between the electron and ion fluxes will occur roughly for this value of the potential. It is important to note that the main balance determining the saturation level is between

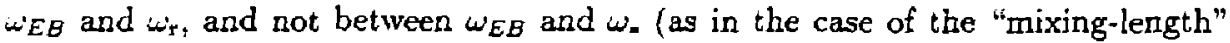
or "local-flattening" estimates, ${ }^{28}$ although for the most unstable modes, $\omega_{r}$ and $\omega$. are of the same order of magnitude). This is because the ratio of $\omega_{E B}$ to $\omega_{\mathrm{r}}$ is what determines the strength of the ion $E \times B$ trapping and hence the ion flux. The saturation level thus predicted is

$$
\phi_{0}=\frac{\dot{\omega}_{r}}{4(1-b) k_{y} \kappa} \simeq \frac{1}{1+k_{\perp}^{2}},
$$

where the linear form of $\omega_{\mathrm{r}}$ has been used to obtain the second expression. The first expression, with the measured value of $\omega_{\mathrm{r}}$ used, agrees very well with the runs of the gyrokinetic code which show the strongest glob formation. Examples are: 
1. a $\theta=0.002, \nu_{\mathrm{ei}}=0.01$, zero ion gyroradius run for which $\omega_{\mathrm{r}} \simeq 0.048$, giving the estimate $\phi_{0} \simeq 0.07$ compared to the observed value of $\phi_{0} \simeq 0.08$,

2. a $\theta=0.002, \nu_{\mathrm{ej}}=0.001, \tau=4, L_{y}=4 L_{x}=128 \Delta$ run for which $\omega_{\mathrm{r}} \simeq 0.015$, giving the estimate $\phi_{0} \simeq 0.10$, in agreement with the observed value to within the $5 \%$ measurement error, and

3. a $\theta=0.002, \nu_{\mathrm{ei}}=0.001, r=100$ run of the linearized $E_{\| \mid}$code described by one of the present authors, ${ }^{19}$ for which $\omega_{r} \simeq 0.065$, giving the estimate $\phi_{0} \simeq 0.09$, again in agreement with the observed value to within measurement error.

For lower values of the collisionality and for $\theta=0.01$, the glob formation is less important than the nonresonant difusion, so Eq. (47) overestimates the saturation level. For example, for the collisionless $\theta=0.002, \nu_{\mathrm{ei}}=0, \tau=4$ case, we observe $\omega_{\mathrm{r}}=0.035$, so this estimate gives $\phi_{0} \simeq 0.07$, compared to the observed value, $Q_{0}=0.04$. Qualitatively, the scaling with $\omega_{r} /(1-b)$ agrees quite well with the comparison between the $\tau=4$ and $\tau=100$ results, and the scaling with $1 /\left(1+k_{\perp}^{2}\right)$ agrees with the comparison of the $32 \times 128$ run with its $32 \times 32$ counterpart.

\section{The $\kappa \rightarrow 0$ Limit}

The question arises as to whether the change in the particle motion due to the added velocity in the nonlinear- $\kappa$ driving term causes significant nonphysical effects in the simulation model. Firstly, the asymmetry due to the $\kappa$ term is unlikely to be the cause of the spatial coherence of our simulations. Such coherent states are observed in runs with $\kappa=0.107$, where the asymmetry due to the $\kappa$ term is weaker, as well as in simulations (both fluid and particle) without such terms. ${ }^{29}$ Secondly, the presence of the coherent component of the particle flux is a consequence of the coherent nature of the fields in the saturated states and not of the asymmetry in the quality of the trapping caused by the $\kappa$ term. On the basis of the arguments macie in the previous subsection, as $\kappa \rightarrow 0$, the $\kappa$ term drops out of Eq. (46) and the saturation siterion becomes $\omega_{\mathrm{r}} / \omega_{E B} \lesssim \min [\epsilon, \xi g(\epsilon)]$, where $\xi<1$ is a factor associated with the strength of the globs required to carry enough flux to cause saturation. Thirdly. in the absence of the nonlinear- $\kappa$ term, self-consistent effects can sill produce an asymmetry in the quality of the $E \times B$ trapping between the potential maxima and minima. The way in which they can arise can be understood as follows. Assume that the electron response is approximately adiabatic. Then from the gyrokinetic Poisson equation, it follows that a strong local maximum in the ion gyrocenter density will produce a local potential maximum. Thus, if an ion glob begins to form at a potential minimum, it will quickly distort the potential contours in such a way as to detrap itself. This effect is evidenced by the fact that 
globs are never observed at the potential minima in the self-consistent simulations. Similarly, a glob formed at a potential maximum will enhance the maximum leading to tighter self trapping. Thus, the additional velocity of the nonlinear- $\kappa$ driving term is not a significant qualitative defect in these simulations. In more turbulent situations, its effects will be even smaller.

\section{E. Saturation Spectra and Energy Exchange}

In this section, we give a brief discussion of the mechanisms determining the wave number spectra of $\phi$ in the steady-state phase. The relationship between the robustness of the spectra and the level of energy exchange will also be briefly commented on. The discussion in this section is somewhat inconclusive and is given primarily to suggest some possible lines of investigation that may clarify the picture.

It is a common feature of the simulations studied here that the spectra are strongly dominated by the longest-wavelength modes of the system $\left[\left(k_{x}, k_{v}\right)=\right.$ $\left.\left(2 \pi / L_{x}, \pm 2 \pi / L_{y}\right)\right]$. Although this dominance would not be expected on the basis of the linear growth rates, it is a quite universal feature of these simulation runs. For $\tau=100$ and $\theta=0.002$, there are many growing modes and, for low $k$, the linear growth rates do not decrease sharply as $k$ increases (provided that $k_{\perp} \rho_{\mathrm{i}} \lesssim 1$ ). The comparison, in Sec. III, between the $32 \times 128$ run with the $32 \times 32$ run shows that the modes that were dominant in the $32 \times 32$ run have much reduced saturation levels in the $32 \times 128$ run, even though the modes which actually dominate the latter case have much lower growth rates.

The initial driving of the lowest modes of the $32 \times 128$ run is most likely due to nonlinear effects, since they are observed to grow very suddenly, with growth rates much larger than predicted by linear theory. This nonlinearity may be a manifestation of a modified two-dimensional fluid inverse energy cascade, due primarily to nonlinear distortion of the ion polarization density by the $E \times B$-flow field. The dominance of the lowest modes in the saturated states may also be due to the combination of the downward-in- $k$ transfer of energy by this process and the sparsity of available modes at low $k$. There are few low- $k$ modes available that can couple to send energy up to the higher- $k$ modes. Thus, the energy transfer in $k$ space favors a "condensation" of the energy in the longest-wavelength modes available in the system. It may be possible to avoid such states by suppressing the lowest modes of the potential so that the lowest available modes more nearly form a continuum. This is left for future studies.

The energy exchange between the + and - modes is due mainly to the interaction between the + and - modes of $\phi$ with the higher modes of the density, through the $\boldsymbol{E} \times \boldsymbol{B}$ nonlinearity and possibly (in the $\theta=0.01$ cases) the parallel velocity-space nonlinearity. The higher modes of the potential are reduced by the 
$1 / k_{\perp}^{2}$ weighting factor from the gyrokinetic Poisson equation, making them less important. In configuration space, these higher modes of the density are the result of nonuniformities at the edges of the globs, particularly for the ions, and of groups of ions and electrons which have just been $E \times B$ detrapped from one cell of the potential and retrapped in an adjacent cell. The $\boldsymbol{E} \times \boldsymbol{B}$ flow of such a group of particles, after the retrapping but before the shear in the flow can spread it out, produces the higher modal components of the density. The $E \times B$ advection of these causes them to contribute, in a time-dependent fashion, to the + and - modes of the densities, and hence, to the potential. It is difficult to make quantitative estimates of the level of this exchange with any accuracy. Some qualitative statements can, however, be made. First, since the energy exchange involves the higher modes of the densities, it is a measure of the stability of the states dominated by the + and - modes. The level of the fluctuations is such that typically the ratio between the maximum and minimum amplitudes due to these fluctuations is about 1.5 , and intermittently may be as high as 2-3. Any larger level of energy exchange would be symptomatic of the breakup of the kind of semi-coherent state observed in the simulations studied here. Secondly, the most collisional $\nu_{e i}=0.01, \theta=0.002$ runs show a gradual decrease in the level of the exchange. This is attributable to the growth and concentration of the globs. As they become more concentrated, the distance from the $O$ point of the velocity field of any raggedness at the boundaries of the glob, and therefore the distance through which the ragged pieces are advected by the $\boldsymbol{E} \times \boldsymbol{B}$ flow, decreases. There are indications from mode-coupling Vlasov code studies by Lee et al. ${ }^{3}$ that the electron parallel acceleration nonlinearity plays a role, although gyrokinetic simulations with this nonlinearity removed ${ }^{19}$ show a comparable or slightly reduced level of energy exchange.

There is a question concerning why the fluctuating + and - modes have equal time-averaged amplitudes instead of forming "streamer" states such as those seen in fluid simulations of the collisional $\eta_{e}$ mode ${ }^{30} \mathrm{~A}$ three-mode analysis along the lines of Drake et al. ${ }^{30}$ with the only nonlinearity present arising from the $E \times B$ advection of the ion polarization density response indicates oscillations in the mode amplitudes about a symmetric state and the absence of an instability that would drive the streamers. This is also consistent with the conclusion from the simulations with the parallel acceleration linearized ${ }^{19}$ that the $\boldsymbol{E} \times \boldsymbol{B}$ nonlinearities dominate the self-consistent dynamics of the energy exchange.

\section{F. Three-Dimensional Effects}

The particle simulation runs considered so far in this paper are "2 $\frac{1}{2}$-dimensional," meaning that for all spatial dependences, $\partial / \partial z=0$. In this system the particle motion is given by a two-dimensional Hamiltonian. For electrostatic drift waves in a three-dimensional shear-free geometry, there is a possibility of significant differences 
in the electron motion due to three-dimensional effects. For parameters comparable to those used here, if the wave number spectrum of $\phi$ is dominated by the lower $k_{\|}$ modes, the ion behavior may still be adequately described by cold fluid equations.

The importance of three-dimensional effects can be shown by considering the following simple generalization of our picture of the two-dimensional electron behavior. We assume that the spectrum of $\phi$ is dominated by four modes of equal amplitudes with wave vectors $\left(k_{x}, k_{y}, k_{z}\right),\left(k_{x},-k_{y}, k_{z}\right),\left(k_{x}, k_{y},-k_{z}\right)$, and $\left(k_{x},-k_{y},-k_{z}\right)$, where $k_{x}, k_{y}$, and $k_{z}$ are fixed positive wave numbers. This generalizes the spectra observed in the $2 \frac{1}{2}$-dimensional runs by symmetrically including modes with negative $\theta$ (i.e., modes with $\theta=-\left|k_{z} / k_{y}\right|$ ). Thus, for illustrative purposes in this paper, we are neglecting the possibilities of spontaneous symmetry breaking or the formation of structures localized in the $z$ direction. If, for the moment, we also neglect the parallel acceleration, then the electron $\boldsymbol{E} \times \boldsymbol{B}$ motion can be understood crudely in terms of the model of Kleva and Drake ${ }^{16}$ and Horton. ${ }^{17}$ The electrons at the positive $v_{\|}$resonance see a stationary potential structure due to positive $\theta$ waves and a moving one due to the negative $\theta$ wave. In the $x-y$ plane the negative $\theta$ wave is moving past the positive $\theta$ wave at twice the phase velocity in the $-y$ direction. This is just the situation considered in these references. Upon writing the stochasticity onset criterion of reference, ${ }^{17}$ for waves of comparable amplitude (noting that Horton's "A's" $=4\left|\phi_{ \pm}\right|$in the notation used here), one obtains $\omega_{E B}>\omega_{\mathrm{r}}$, where $\omega_{E B}=4 k_{x} k_{y}\left|\phi_{ \pm}\right|$is the $\boldsymbol{E} \times \boldsymbol{B}$ trapping frequency due to waves of a single $\theta$ (positive, say). For a typical saturation level of the $2 \frac{1}{2}$-dimensional runs, $\left|\phi_{ \pm}\right| \simeq 0.04$ and $\omega_{\mathrm{r}} \simeq 0.05$ we have $\omega_{E B} \simeq 0.1$, so the electron orbits are stochastic by this criterion.

When weak parallel acceleration is included, as in the $\theta=0.002$ runs, the resonance overlap criteria of Horton ${ }^{17}$ and Kleva and Drake ${ }^{16}$ become the criteria for the elimination of the resonance depopulation seen in the $2 \frac{1}{2}$-dimensional runs. This is just the statement that if the resonances associated with the positive and negative $\theta$ waves overlap, then the electrons can no longer undergo resonant parallel acceleration due to just the positive $\theta$ or just the negative- $\theta$ waves. We have verified this simple intuition by modifying the non-self-consistent code of Sec. $V$ to include both the positive and negative- $\theta$ waves. The detailed results will be presented elsewhere. If these spatial three-dimensional effects are important in three-dimensional gyrokinetic code runs, then the dependences of the fluxes and the saturation levels on the collisionality would be expected to be weaker. Some preliminary three-dimensional gyrohinetic simulations of drift waves have been reported, ${ }^{20}$ although more detailed diagnostics are needed before any precise statements about the particle dynamics in these can be made. The above discussion indicates, however, that the understanding of the individual resonances in the $2 \frac{1}{2}$-dimensional runs is likely to be of great importance to the understanding of the multiple-resonance situations that will occur in three-dimensional geometries. 


\section{CONCLUSIONS AND SUGGESTIONS FOR FURTHER WORK}

In this paper, we have investigated the nonlinear dynamics of electrostatic collisionless and weakly collisional drift waves in a shear-free slab geometry. The gyrokinetic particle simulations studied here are the first simulations of gradientdriven microinstabilities that treat the nonlinear dynamics in a fully self-consistent manner and which made possible the study of the evolution well into a steady-state post-saturation phase. This paper reports the first detailed consideration of the combined effects of $E \times B$ trapping in configuration space coupled with parallel acceleration in velocity space and dissipation on the nonlinear evolution observed in these simulations. Such an approach was necessary to explain the behavior of the particle flux. A useful spinoff from this work was the construction of Hamiltonians for quite a wide class of $2 \frac{1}{2}$-dimensional non-self-consistent gyrocenter systems.

The main results concerning the nonlinear dynamics of the $2 \frac{1}{2}$-dimensional simulations are as follows. The saturation levels for the potential are determined by the requirement that the values of the non-self-consistent electron and ion fluxes, as a function of the value of the potential, balance. The minimum value of the electron flux is set by the $E \times B$ diffusion of the parallel resonant electrons. This has a roughly quadratic dependence of the saturation level for the potential. The ion flux may be due either to the coherent advection of $E \times B$-trapped high-density regions or to $E \times B$ diffusion. The high-density regions are the result of outward advection of very well $E \times B$-trapped regions of ions. Both of these mechanisms switch on quite abruptly as a function of the saturation level above a certain value. This is because the ion parallel velocity is negligible so that all of the ions are brought into resonance with the waves by nonlinear effects at the same threshold amplitude, while the number of electrons in resonance increases smoothly with the width of the resonance. The non-self-consistent electron flux has a parametric dependence on the pitch-angle scattering and the inclination angle $\theta$ between the wavefronts and the magnetic field. This dependence arises because in the $2 \frac{1}{2}$-dimensional geometry the resonant electrons, which diffuse the most, are accelerated out of the resonance by the parallel electric field of the waves, thereby decreasing their diffusion. The collisions increase the flux by repopulating the resonance. The flux decreases as $\theta$ is increased, since increasing $\theta$ increases the effectiveness of the parallel acceleration compared to the $E \times B$ drift which causes the difiusion.

The relevance of the results for drift waves in the $2 \frac{1}{2}$-dimensional geometry to the three-dimensional geometry is as follows. For simulation parameters similar to those used here, the cold fluid description of the ion dynamics is likrly to be valid if low- $k_{\text {ll }}$ modes dominate the fluctuation spectrum (consistent with their linear growth rates). The presence of multiple resonances can, however, cause the electron 
gyrocenter motion to become chaotic even in the absence of fluctuations in the mode amplitudes of the potential. The main effect of this is to reduce the resonance depopulation due to the parallel acceleration. The $2 \frac{1}{2}$-dimensional simulations provide an understanding of a single resonance.

The Hamiltonians obtained in this paper for the gyrocenter motion in the 2 $\frac{1}{2}$-dimensional geometry, including parallel acceleration, will be of utility for understanding the dynamics of systems in which both $E \times \boldsymbol{B}$ and parallel trapping are involved. One example is the gyrokinetic simulation of $\eta_{i}$ modes. Hamiltonians were derived which include the effect of shear. The Hamiltonians may also be useful for understanding three-dimensional effects such as the presence of multiple and overlapping resonances.

There are several areas of exploration suggested by the results of this paper. We list some of the most immediate.

- A careful treatment of the stochasticity of the ion orbits to understand the functional dependences of the quality of the $E \times B$ trapping near oscillating $O$ points, in particular to estimate analytically the extent of the glob formation, would be of interest.

- The issue of whether or not the $O$ point shifts due to the nonlinear- $\kappa$ term are an important factor in the self-consistent evolution has not been conclusively settled. The problems with the linearized- $k$ codes would seem to prevent their use for this study. ${ }^{19}$ The most promising way to proceed seems to be to make runs with smaller values of $\kappa / k_{x}$ for the dominant modes.

- Finally, the ongoing development of gyrokinetic particle simulations will no doubt result in a hierarchy of more and more realistic simulations. The understanding gained in each stage will no doubt contribute towards the understanding of the next. The simulations of the $\eta_{\mathrm{i}}$ mode already done by Lee and co-workers ${ }^{7}$ form a part of such a hierarchy and also indicate that many of the ideas relevant to the understanding of the collisionless drift waves will carry over to that problem. There are thus interesting questions concerning the similarity and differences between the nonlinear dynamics of drift waves of the kind studied here and $\eta_{\mathrm{i}}$ modes, both in the $2 \frac{1}{2}$ - and three-dimensional geometries.

\section{ACKNOWLEDGMENTS}

The authors wish to thank J. Krommes, C. Oberman, R. Sydora, and T. S. Hahm for many useful discussions.

This work was supported by the U.S. Department of Energy, partly through Contract Nos. DE-ACO2-76-CHO-3073 and DE-FG05-86ER53221. 


\section{References}

${ }^{1}$ A. Hasegawa and K. Mima, Phys. Fluids 21, 87 (1978).

${ }^{2}$ J. F. Federici, W. W. Lee, and W. M. Tang, Phys. Fluids 30, 425 (1986).

${ }^{3}$ W. W. Lee, J. A. Krommes, C. Oberman, and R. A. Smith, Phys. Fluids 27, 2652 (1984).

${ }^{4}$ P. C. Liewer, Nucl. Fusion 25, 543 (1985).

${ }^{5}$ W. W. Lee, J. Comp. Phys. 72, 243 (1987).

${ }^{6}$ W. W. Lee, Phys. Fluids 26, 556 (1983).

'W. W. Lee and W. M. Tang, Phys. Fluids 31, 612 (1988).

${ }^{8}$ T. Antonsen, B. Coppi, and R. Englade, Nucl. Fusion 19, 64I (1979).

${ }^{9}$ T. H. Dupree, Phys. Fluids 10, 1049 (1967).

${ }^{10} \mathrm{P}$. W. Terry and W. Horton, Phys. Fluids 26, 106 (1983).

${ }^{1}$ W. Horton, Phys. Fluids 29, 1491 (1986).

${ }^{12}$ R. E. Waltz, Phys. Fluids 26, 169 (1983).

${ }^{13}$ R. Z. Sagdeev and A. A. Galeev, Nonlinear Plasma Theory (W. A. Benjamin Inc., New York, Amsterdam, 1969).

${ }^{14} \mathrm{H}$. Ching, Phys. Fluids 16, 130 (1973).

${ }^{15}$ S. P. Hirshman, Phys. Fluids 23, 562 (1980).

${ }^{16}$ R. G. Kleva and J. F. Drake, Phys. Fluids 27, 1686 (1084).

${ }^{17}$ W. Horton, Plasma Phys. 27, 937 (1085).

${ }^{18}$ R. A. Smith, J. A. Irrommes. and W. W. Lee, Phys. Fluids 28, 1068 (1985).

19. A. M. Dimits, Ph.D. thesis, Princeton Lniversity, 1988.

${ }^{20}$ R. D. Sydora, W. W. Lee, T. S. Hahm, H. Natiou. J. M. Dawson, and Y. K. Decyk, Bull. Am. Phys. Soc. 32, 1889 (1987, paper TT5).

${ }^{21}$ D. H. E. Dubin, J. A. Krommes, C. Oberman, and W. W. Lee, Phys. Fluids 26, 3524 (1983). 
${ }^{22}$ J. A. Krommes, W. W. Lee, and C. Oberman, Phys. Fluids 29, 2421 (1986).

${ }^{23}$ R. Shanny, J. M. Dawson, and J. M. Greene, Phys. Fluids 10, 1281 (1967).

${ }^{24}$ A. Lenard and 1. B. Bernstein, Phys. Rev. 112, 1456 (1958).

${ }^{25}$ W. W. Lee and H. Okuda, in Proceedings of the International Conference on Plasma Physics (Fusion Research Association of Japan, Nagoya Japan, 1980), Vol. 2, p. 105.

${ }^{26}$ W. W. Lee, Y. Y. Kuo, and H. Okuda, Phys. Fluids 21, 617 (1978).

${ }^{27} \mathrm{~A}$. Boozer and G. Kuo-Petravic, Phys. Fluids, 24, 851 (1981).

${ }^{28}$ See for example, W. M. Tang, Nucl. Fusion 18, 1083 (1978).

${ }^{29}$ C. E. Seyler, Y. Salu, D. Montgornery, and G. Knorr Phys. Fluids 18, 803 (1975).

${ }^{30}$ J. F. Drake, P. N. Guzdar, and A. B. Hassam, Phys. Rev. Lett. 19, 2205 (1988). 


\section{Tables}

TABLE I. $\left\{|\phi(k)|^{2}\right\rangle$ for $\theta=0.002$, and $\tau=100$, accurate to within a factor of 2 .

\begin{tabular}{lccccc}
\hline$m \backslash n$ & 0 & 1 & 2 & 3 & 4 \\
\hline 0 & - & - & - & - & - \\
1 & - & $3 \times 10^{-3}$ & $10^{-5}$ & $10^{-6}$ & $5 \times 10^{-8}$ \\
2 & $2 \times 10^{-4}$ & $10^{-5}$ & $5 \times 10^{-6}$ & $2 \times 10^{-7}$ & $10^{-8}$ \\
3 & - & $10^{-6}$ & $2 \times 10^{-7}$ & $2 \times 10^{-8}$ & $10^{-9}$ \\
4 & - & $3 \times 10^{-8}$ & $10^{-8}$ & $2 \times 10^{-9}$ & $3 \times 10^{-10}$ \\
\hline
\end{tabular}

TABLE II. $\left\langle\left|n^{e}(k)\right|^{2}\right\rangle$ and \langle|$\left.\left.n^{i} l\right)\left.\right|^{2}\right\rangle$ for the same run as in Table I.

\begin{tabular}{cccc}
\hline$m \backslash n$ & 0 & 1 & 2 \\
\hline 0 & - & - & $10^{-2}$ \\
1 & - & $10^{-2}$ & $10^{-3}$ \\
2 & $10^{-2}$ & $10^{-3}$ & $3 \times 10^{-3}$ \\
\hline
\end{tabular}

\begin{tabular}{lccc}
\hline$m \backslash n$ & 0 & 1 & $\overline{2}$ \\
\hline 0 & - & - & $2 \times 10^{-2}$ \\
1 & - & $10^{-1}$ & $3 \times 10^{-3}$ \\
2 & $3 \times 10^{-2}$ & $3 \times 10^{-3}$ & $10^{-2}$ \\
\hline
\end{tabular}




\section{Figures}

FIG. 1. (a) Density perturbation in the multiscale model. (b) Density perturbation due to the incompressible advection of nonuniform density profile.

FIG. 2. $2 \frac{1}{2}$-dimensional shear-free slab geometry.

FIG. 3. Time evolution of $(1,1)$ and $(1,-1)$ modes of $\phi$ for $\theta=0.002, \nu_{\mathrm{ei}}=0.001$, and $\tau=100$.

FIG. 4. Time evolution of the particle flux for the same parameter values as for Fig. 3.

FIG. 5. Potential contours from the run of Fig. 3 at (a) $t=2180$, and (b) $t=2070$. The solid contours are positive and dotted contours are negative.

FIG. 6. (a) Electron and ( $b$ ) ion scatter plots at $t=2180$ from the run of Fig. 3 .

FIG. 7. Time histories of $\phi_{+}$and of the electron flux from a run with the same parameter values as for $F$ ig. 3, but with the nonlinear terms in $\Psi$ and in the Poisson equation removed and the ion parallel velocities set to zero.

FIG. 8. Qualitative behavior of electron and ion fluxes as a function of the saturation level.

FIG. 9. Time history of $\phi_{+}(t)$ used in the non-self-consistent simulations.

FIG. 10. Jon gyrocenters from a non-self-consistent simulation with $k_{x} v_{x}=0.0036$ at $t=2000$.

FIG. 11. Ion gyrocenters from a non-self-consistent simulation with $k_{x} v_{x}=0.0$ at $t=2000$.

FIG. 12. Time history of ion gyrocenter flux from a non-self-consistent simulation with $k_{x} v_{x}=0.0036$.

FIG. 13. Time history of ion gyrocenter flux from a non-self-consistent simulation with $k_{x} v_{x}=0.0$

FIG. 14. Spatial distribution of ion gyrocenters for a non-self-consistent run corresponding to $\tau=4$ gyrokinetic run ( $\phi_{0}$ half of value in Fig. 9).

FIG. 15. Electron gyrocenter spatial distribution from a non-self-consistent code run with parameters chosen to model $\theta=0.002 . \nu_{\mathrm{ej}}=0.0 U_{1}, \tau=100$ run of full code. 
FIG. 16. Electron gyrocenter velocity distribution for the same run as in Fig. 15.

FIG. 17. Time histories of electron gyrocenter flux from the same run as for Fig. 15: (a) raw and (b) averaged over $\Delta t=100$ bins.

FIG. 1S. Time history of electron gyrocenter parallel momentum from the same run as for Fig. 15.

FIG. 19. Parallel velocity distribution for the $\nu_{\mathrm{ei}}=0$ run of test electron gyrocenter code.

FIG. 20. Time history of electron gyrocenter parallel momenturn from a non-selfconsistent-code run with $v_{x}=0$, but otherwise the same parameter values as for Fig. 15.

FIG. 21. (a) Instantaneous ion gyrocenter streamlines for weak electric field (no $E \times B$ trapping) with $\kappa=0.214_{\text {-and }} \phi_{+}=\phi_{-}$. (b) Instantaneous ion gyrocenter streamlines for a marginally noulinear case. (c) Instantaneous ion gyrocenter streamlines for for a case with strong $E \times B$ trapping.

FIG. 22. Contours of $\hat{\psi}$ of Eq. (28b) for $\epsilon=0$ (solid), $\epsilon=-0.3$ (dotted), and $\epsilon=0.3$ (dashed).

FIG. 23. Contours of $H$ from Eqs. (28a) and (28b) corresponding to the $\hat{\psi}$ contours of Fig. 5.

FIG. 24. Instantaneous electron gy iocenter streamlines [contours of $H$ from Eq. (31) with the potential of Eq. (19a)] for $\theta=0.01$, for $\epsilon=0$ (solid), 0.3 (dotted), and 0.3 (dashed).

FIG. 25. Instantaneous electron gyrocenter streamlines for $\theta=0.002$, for $\bar{C}_{1}=0$ (solid), 2 (dotted), and 4 (dashed).

FIG. 26. Instantaneous electron gyrocenter streamlines for $\theta=0.01, \bar{C}_{1}=0$ (solid), -0.2 (dashed), and +0.2 (dotted).

FIG. 27. Relaxed density profile for $\phi_{+}=\phi_{-}=$const.

FIG. 28. Grid of $X$ points for parallel resonant and nonresonant $E \times B$ orbits.

FIG. 29. Effect of energy exchange $(\epsilon<0$ case shown) between the + and modes on a least-trapped trajectory. The nonresonant trajectory $A$ has no average $x$ displacement while the resonant trajectory $\mathrm{B}$, averaged over many wavelengths, follows $x-y=$ const. 
FIG. 30. Subdivision of passing area and passing trajectory used for perturbative evaluation of correction to diffusion coefficient.

FIG. 31. Diagram of depletion of parallel velocity distribution function set by balance between parallel acceleration and collisions.

FIG. 32. Time-averaged phase relationship between the + and - modes of $\phi, n^{\mathbf{i}}$, and $n^{e}$. 
(a)

\# $88 \times 0234$

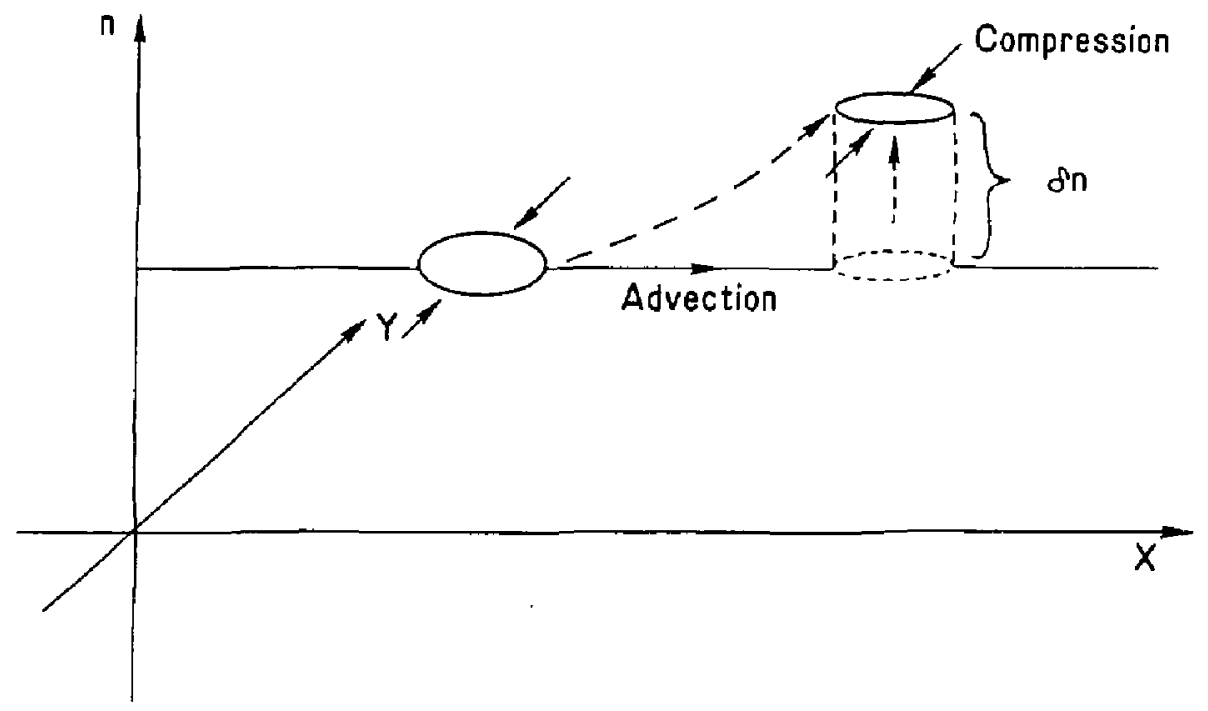

(b)

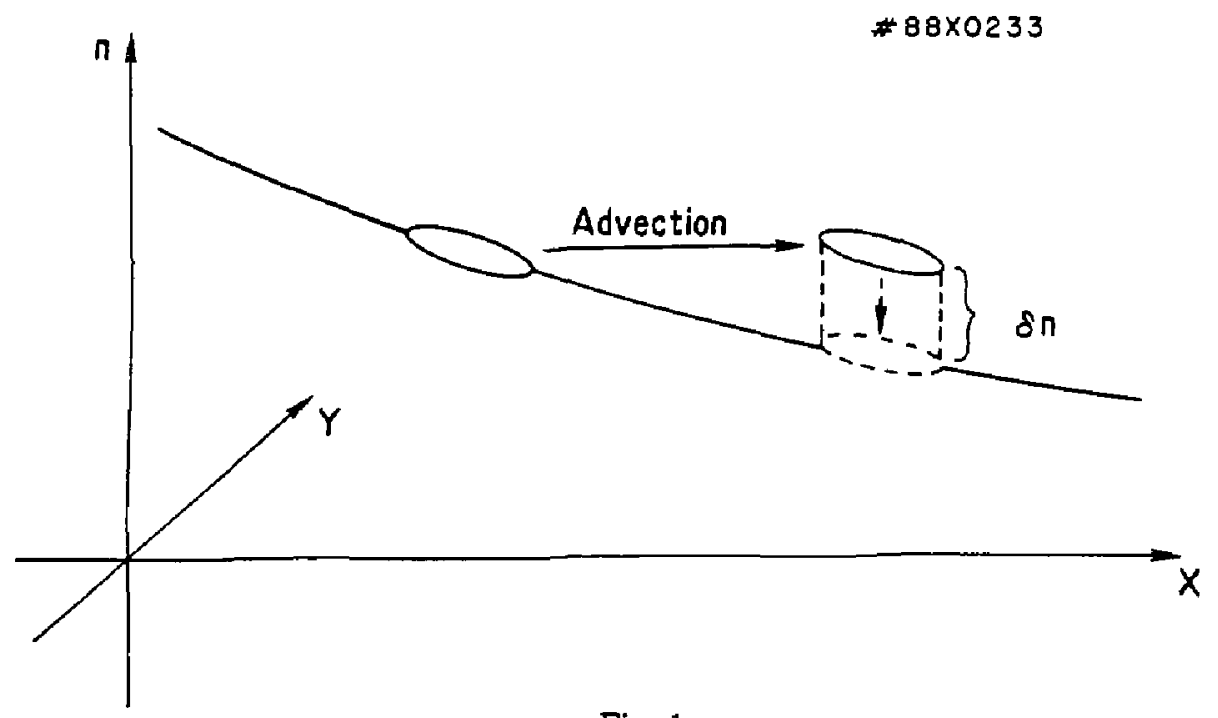

Fig. 1 
*88X0066

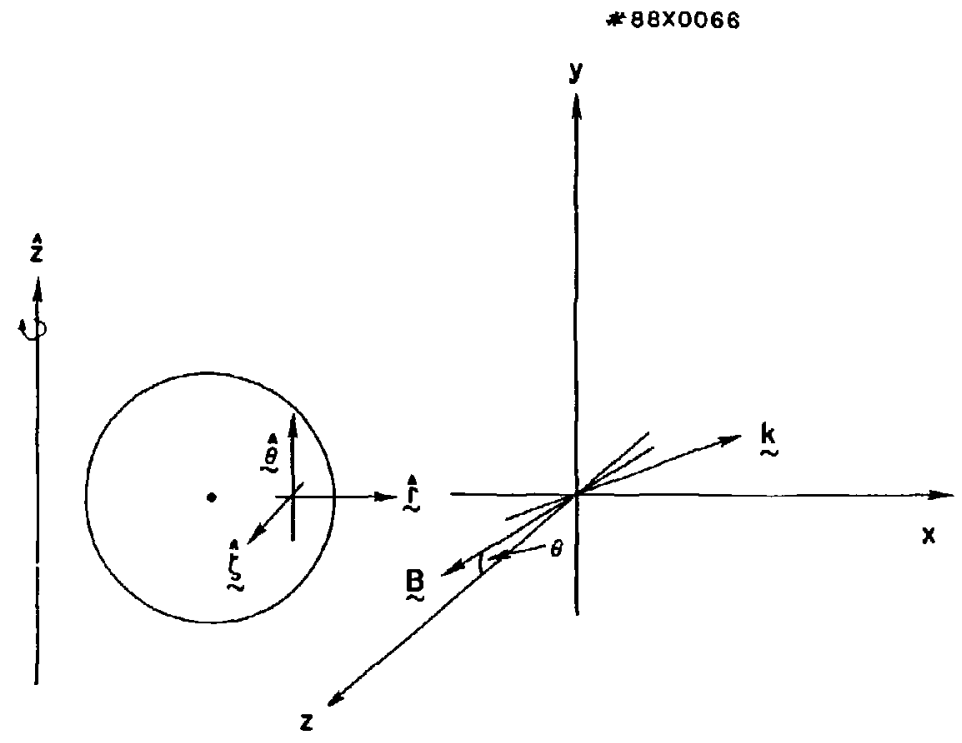

Fig. 2 

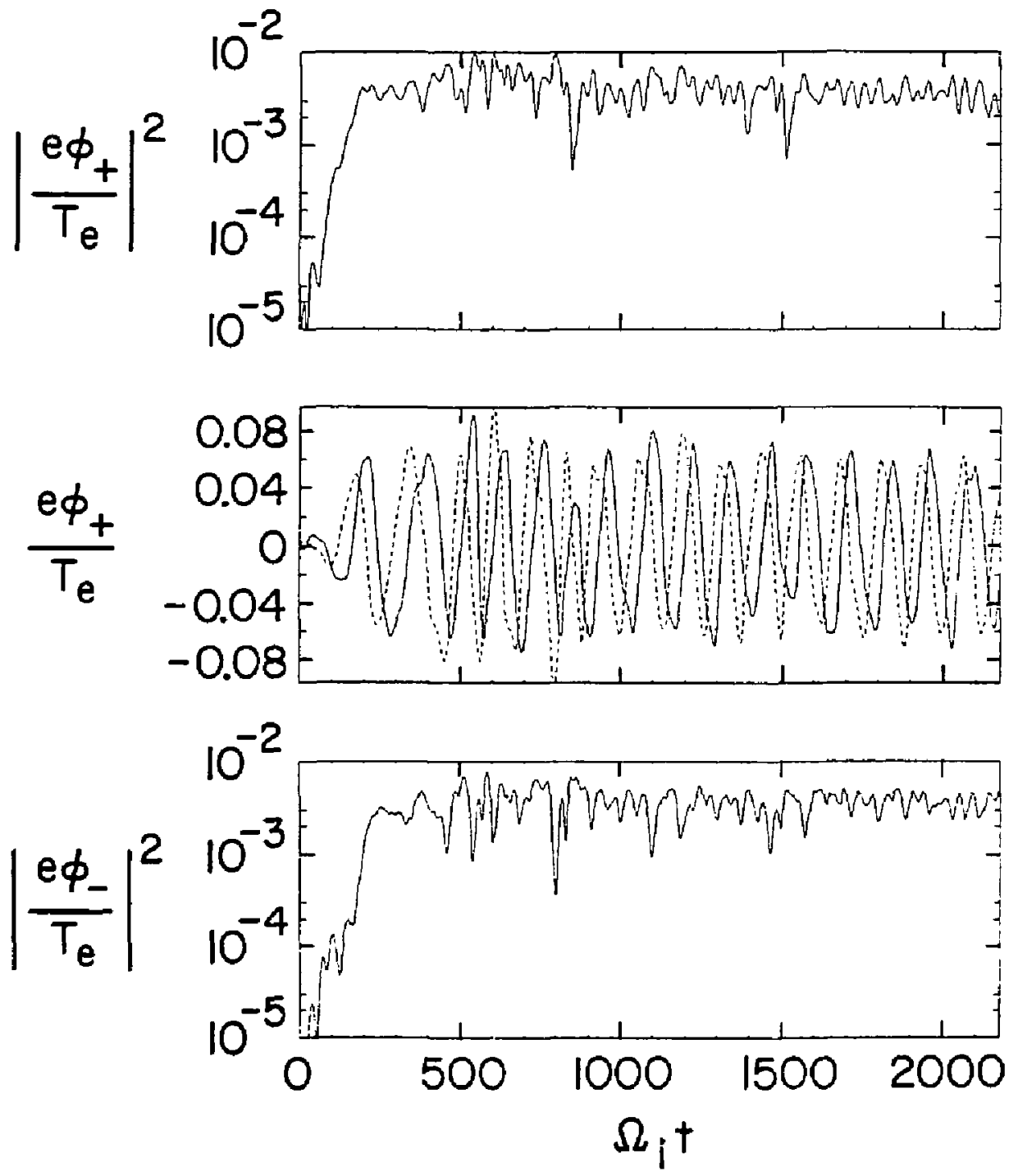

Fig. 3 


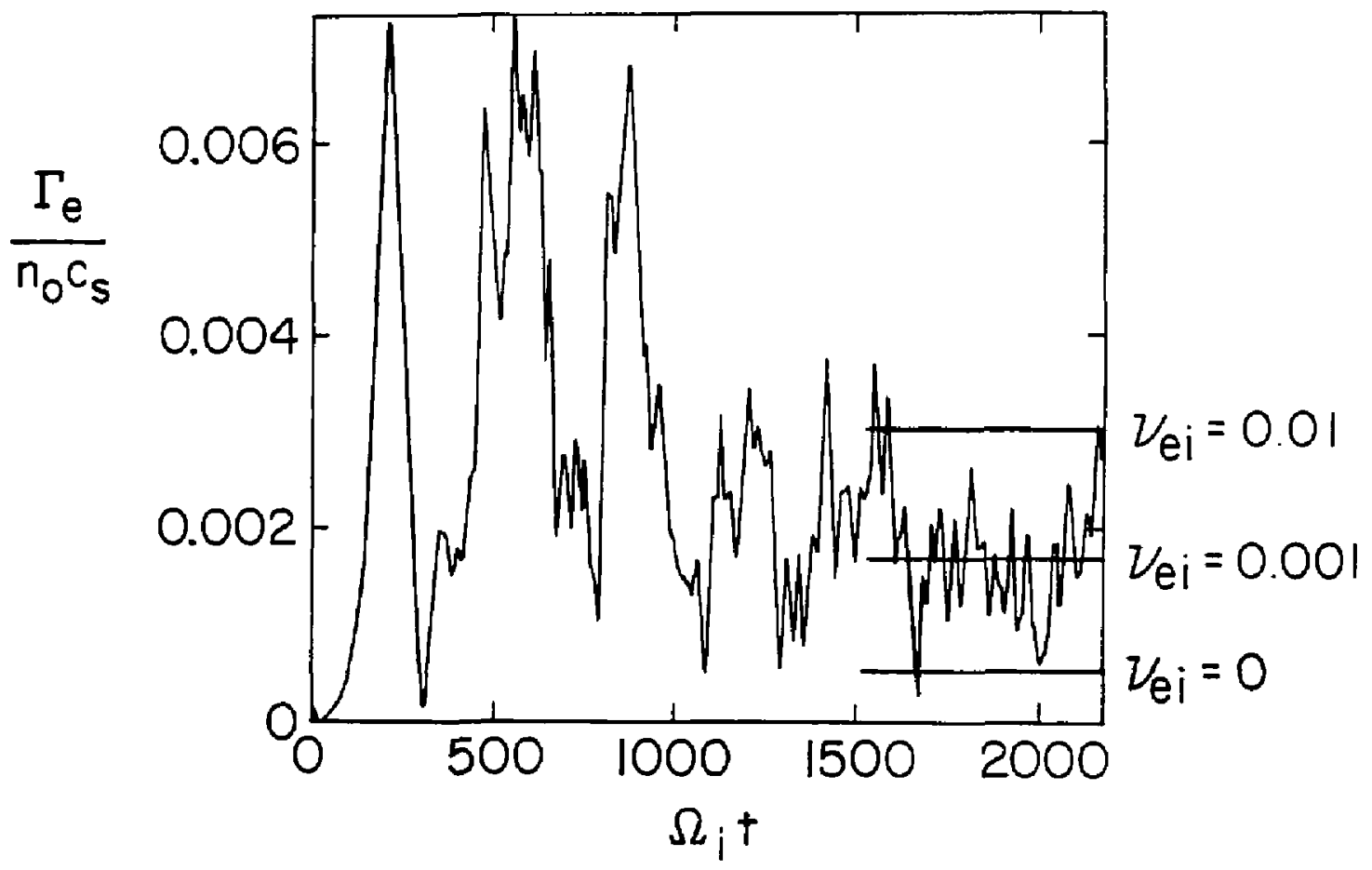

Fig. 4 
(a)

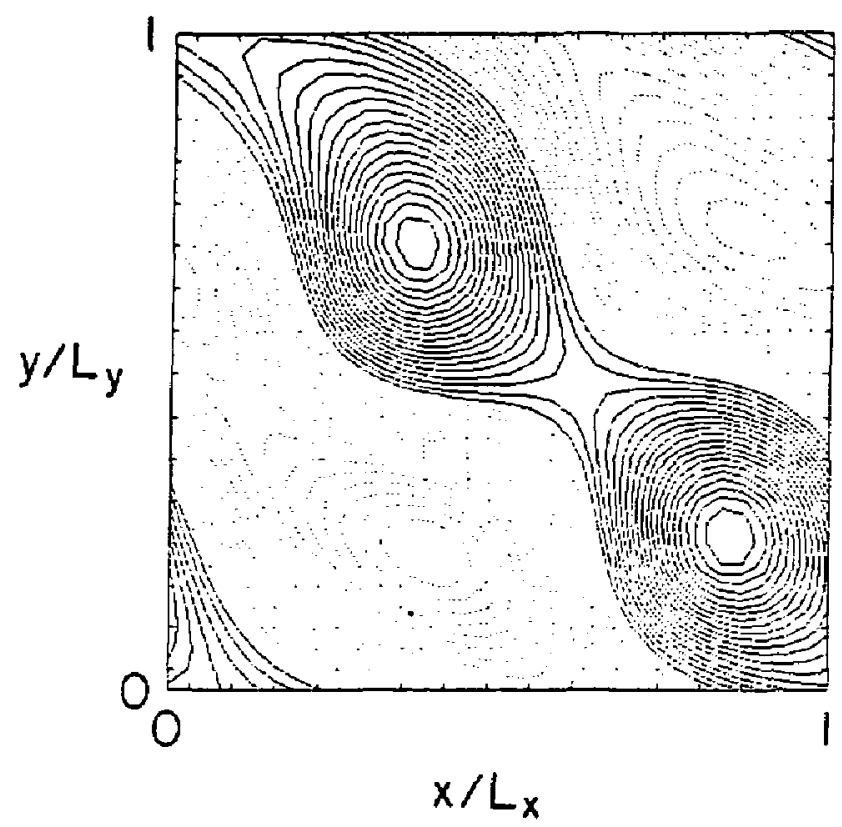

(b)

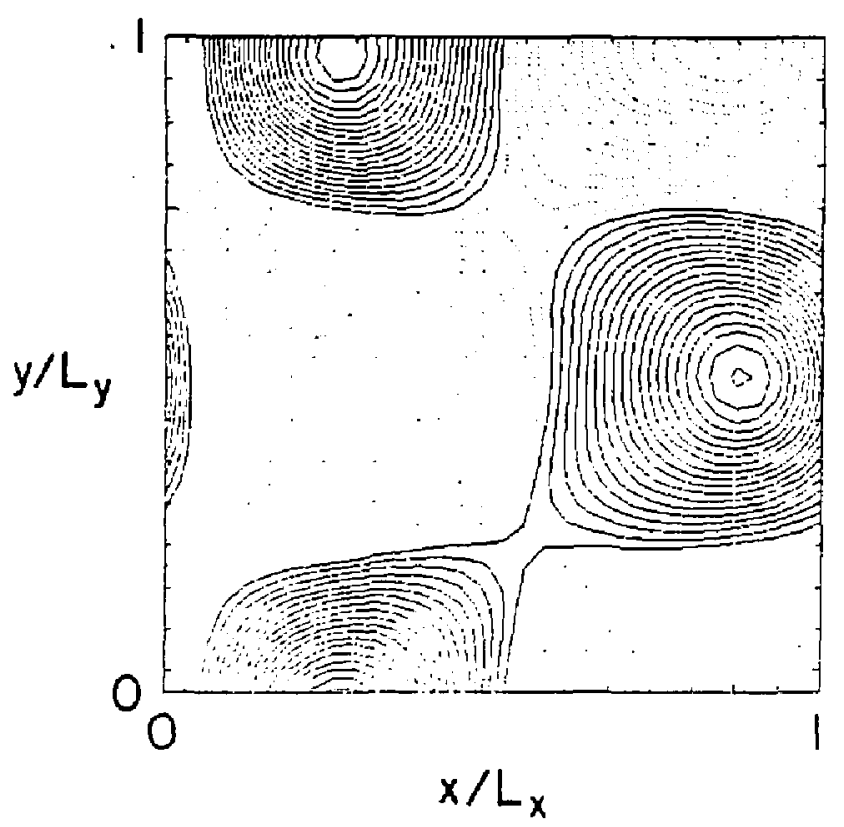

Fig. 5 
(a)

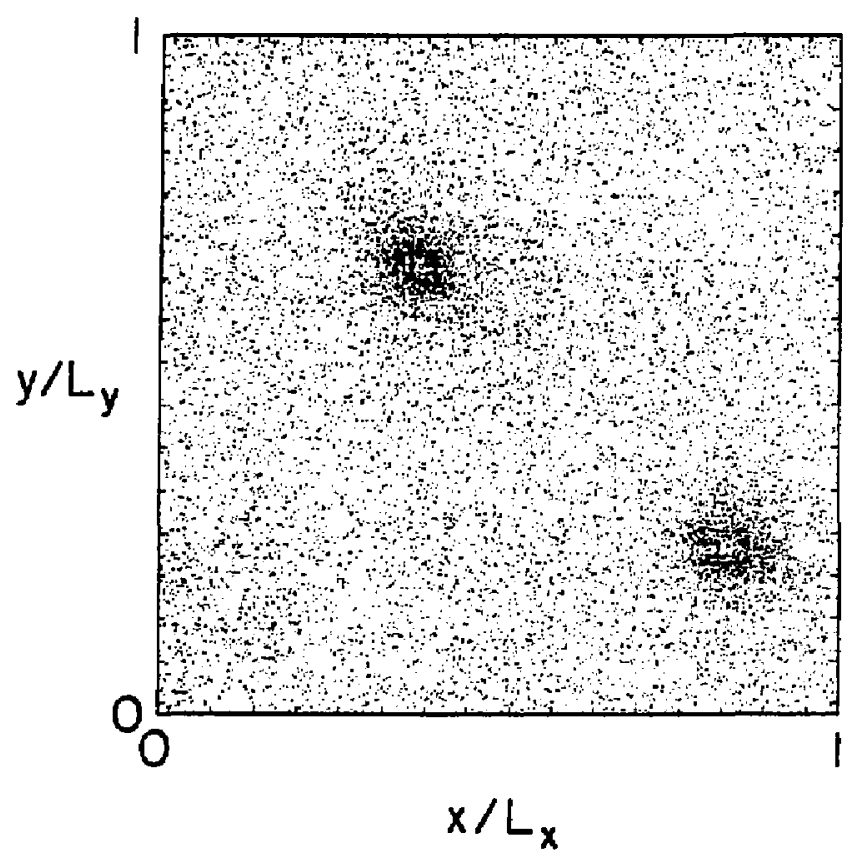

(b)

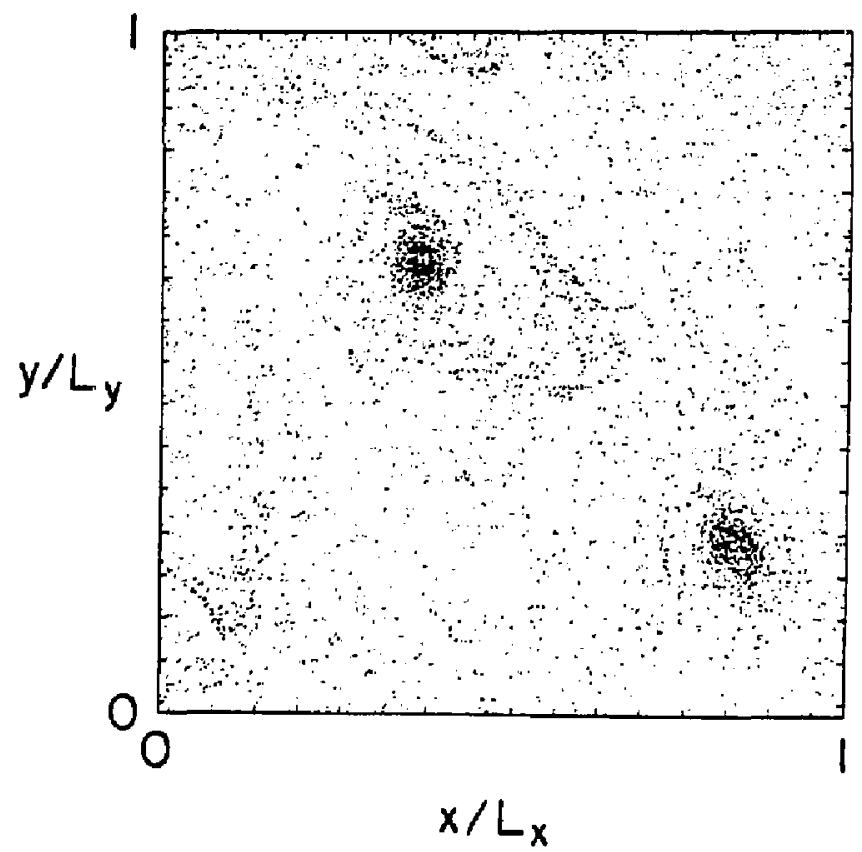

Fig. 6 

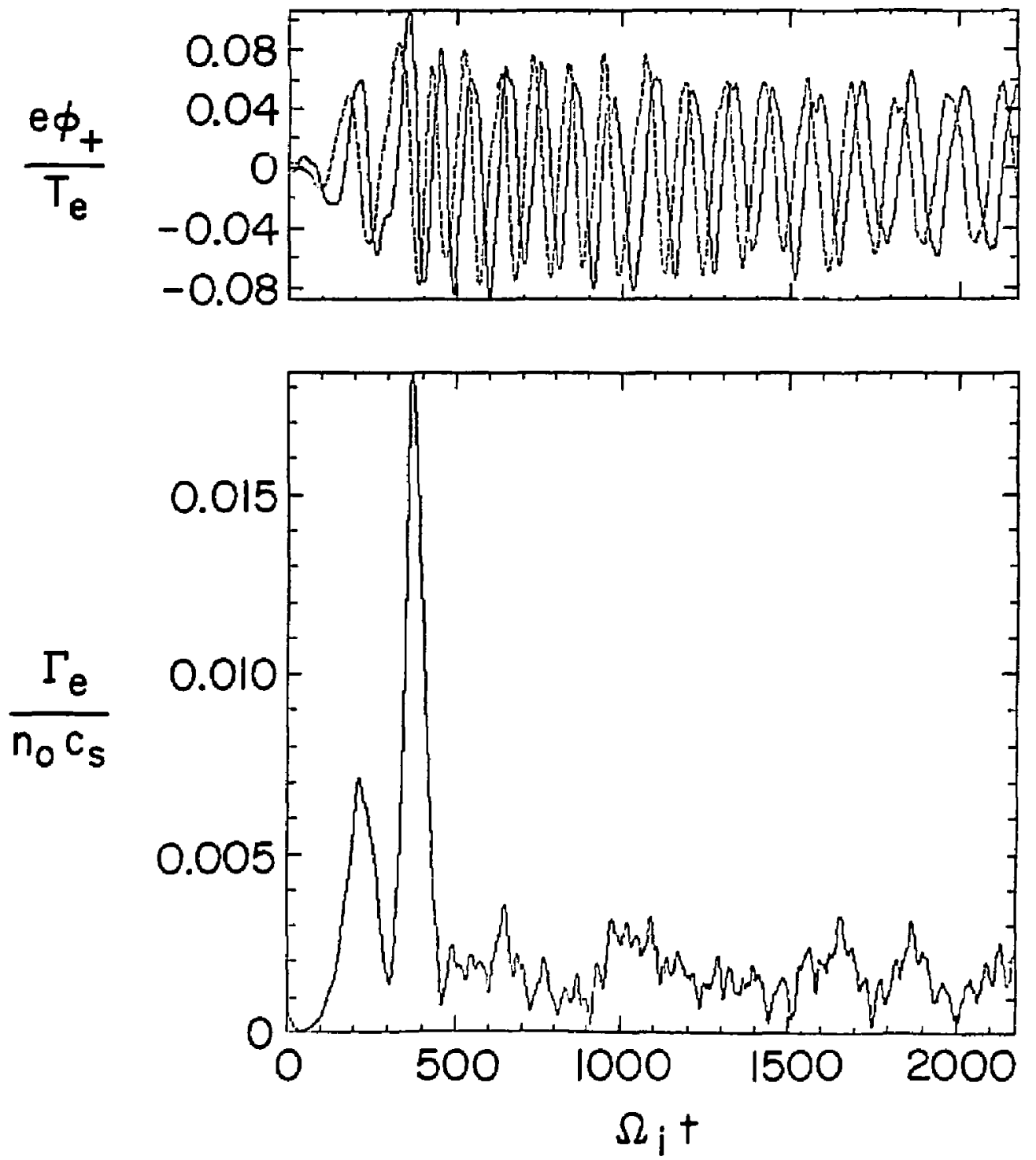

Fig. 7 


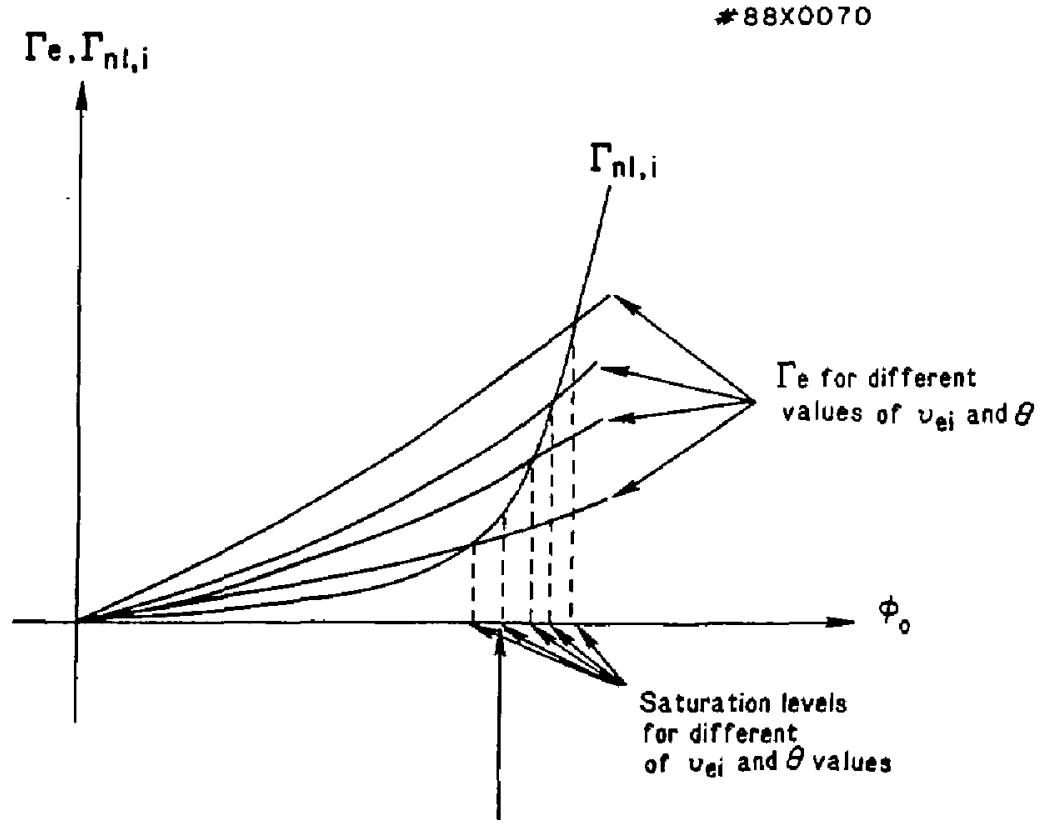

Onset of resonant ion flux and glob-formation

Fig. 8 


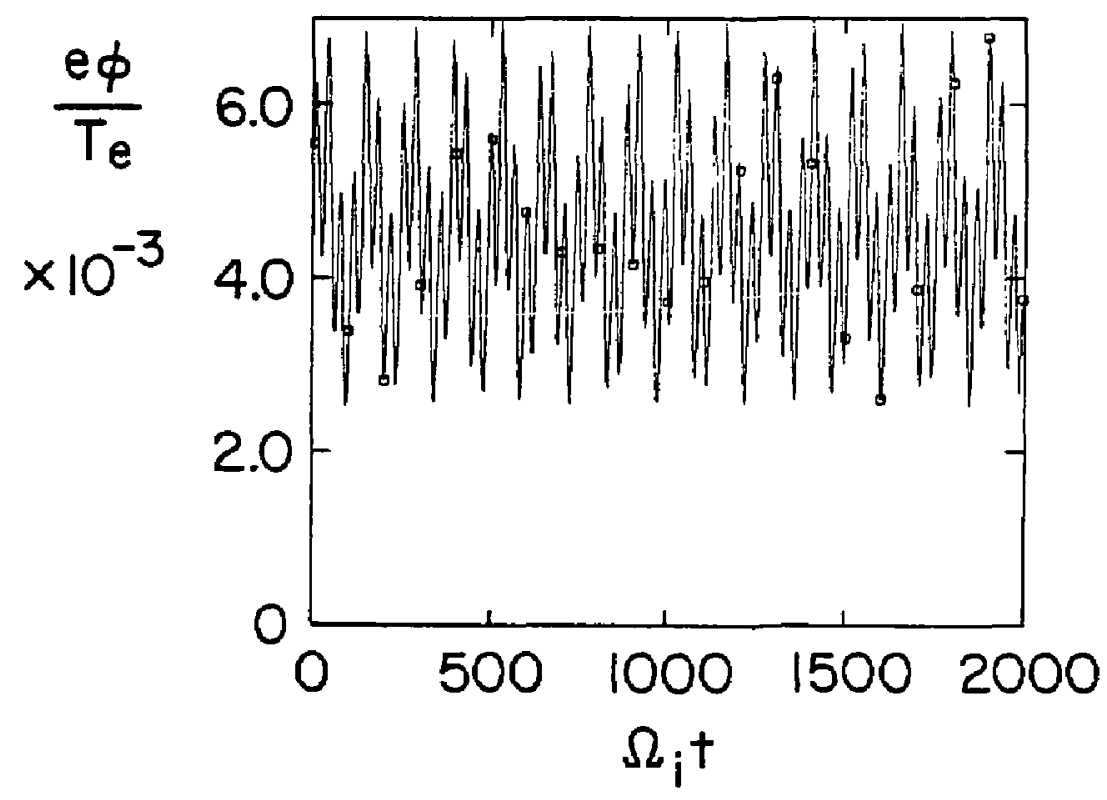

Fig. 9 


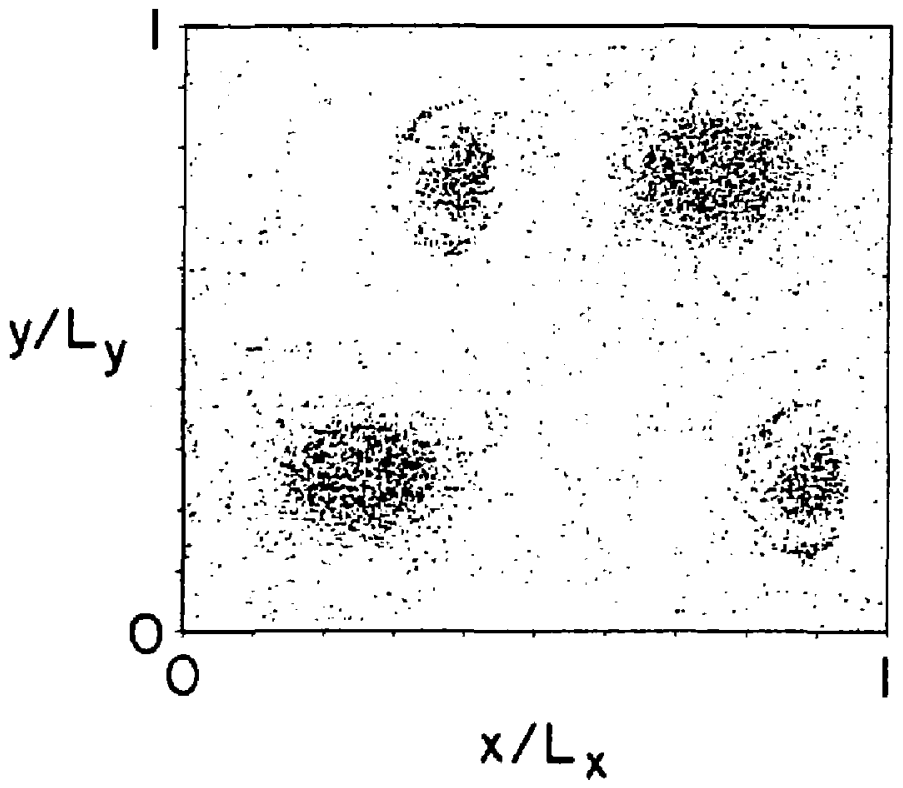

Fig. 10 


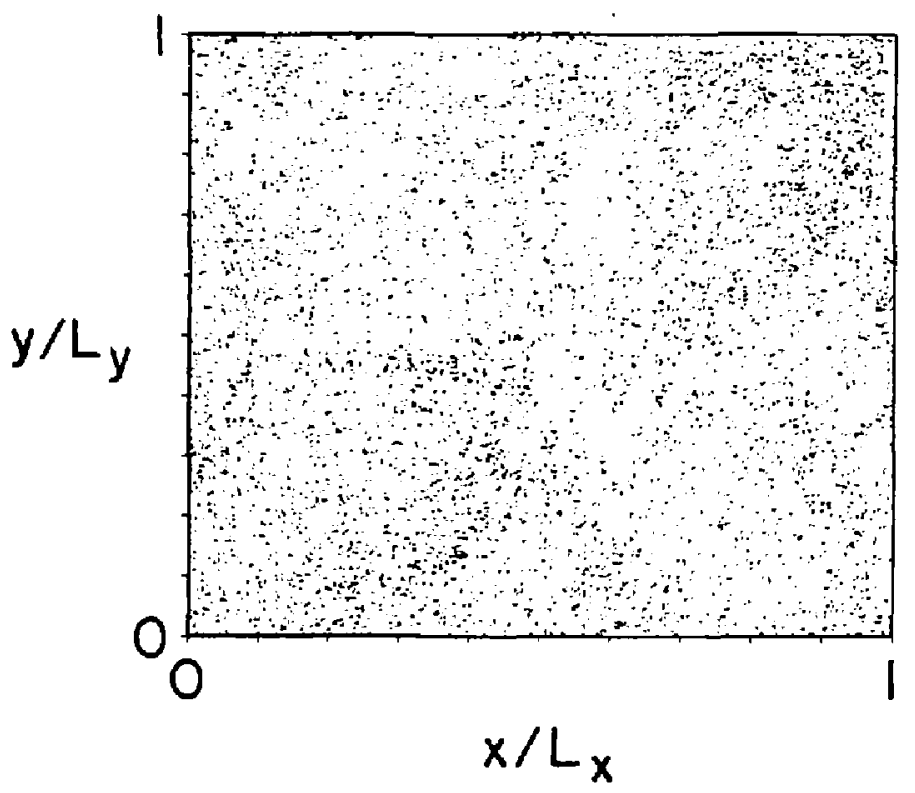

Fig. 11 


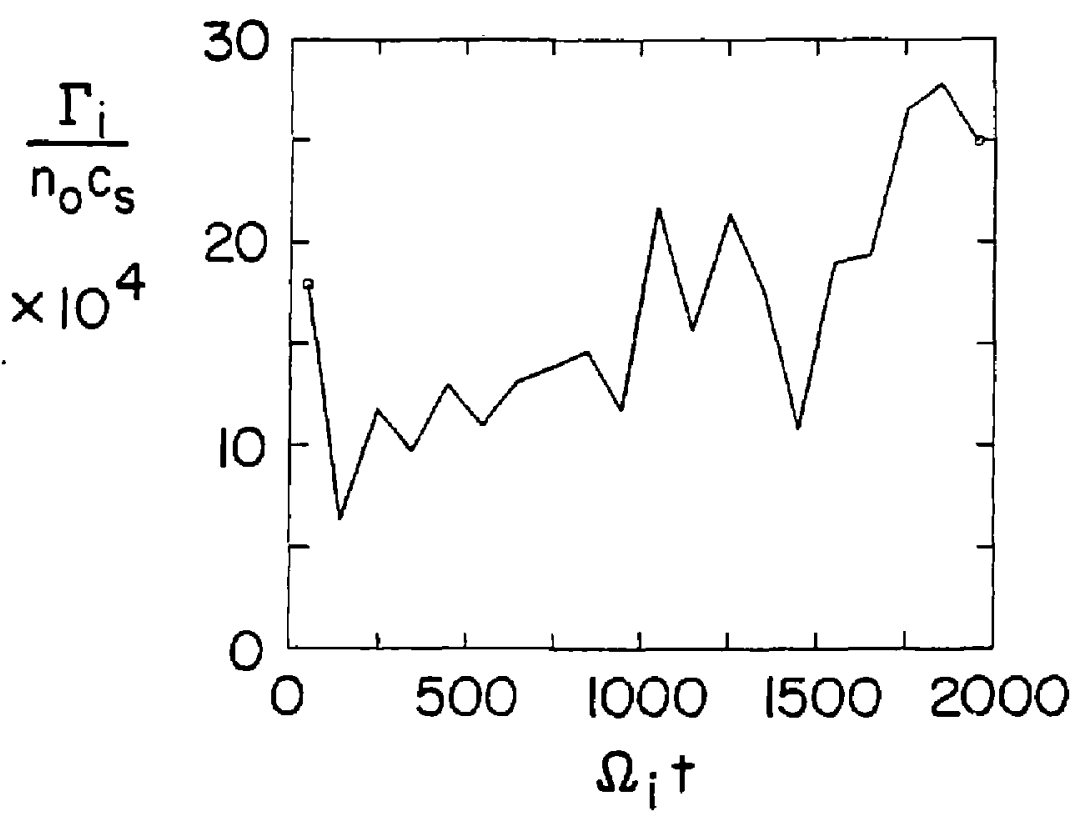

Fig. 12 


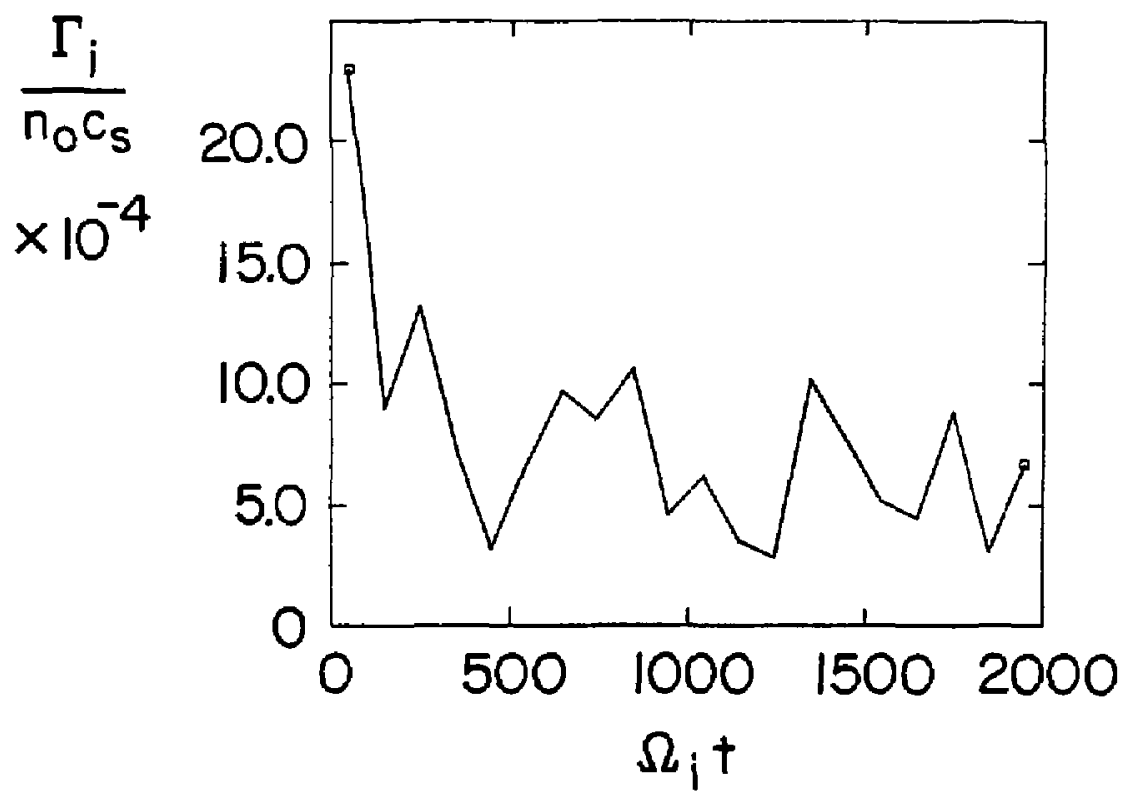

Fig. 13 


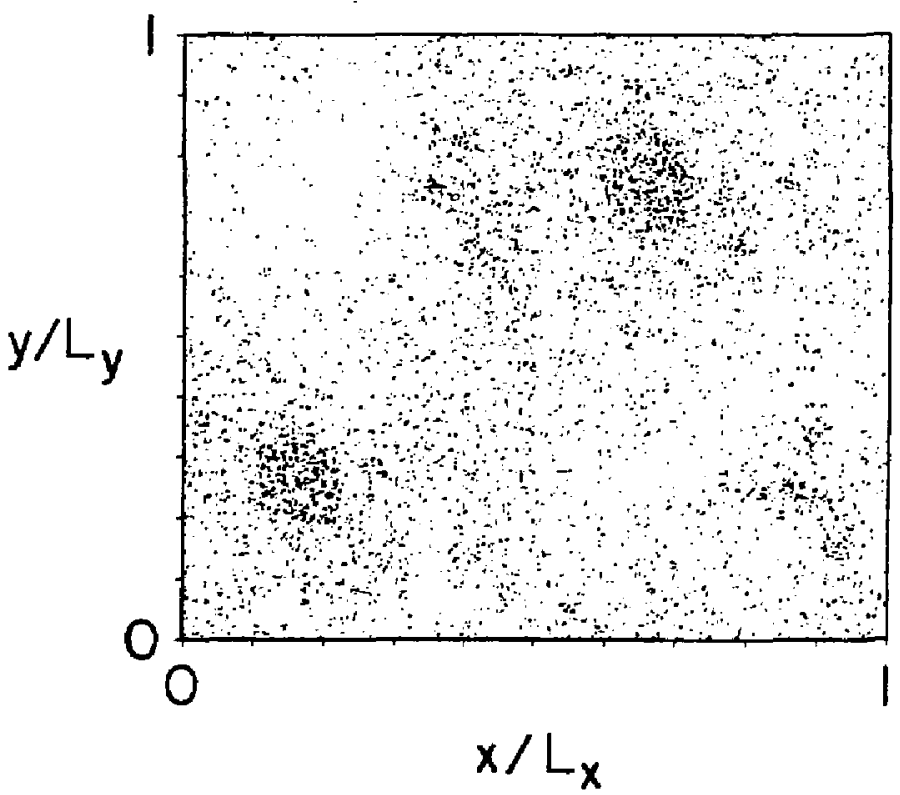

Fig. 14 


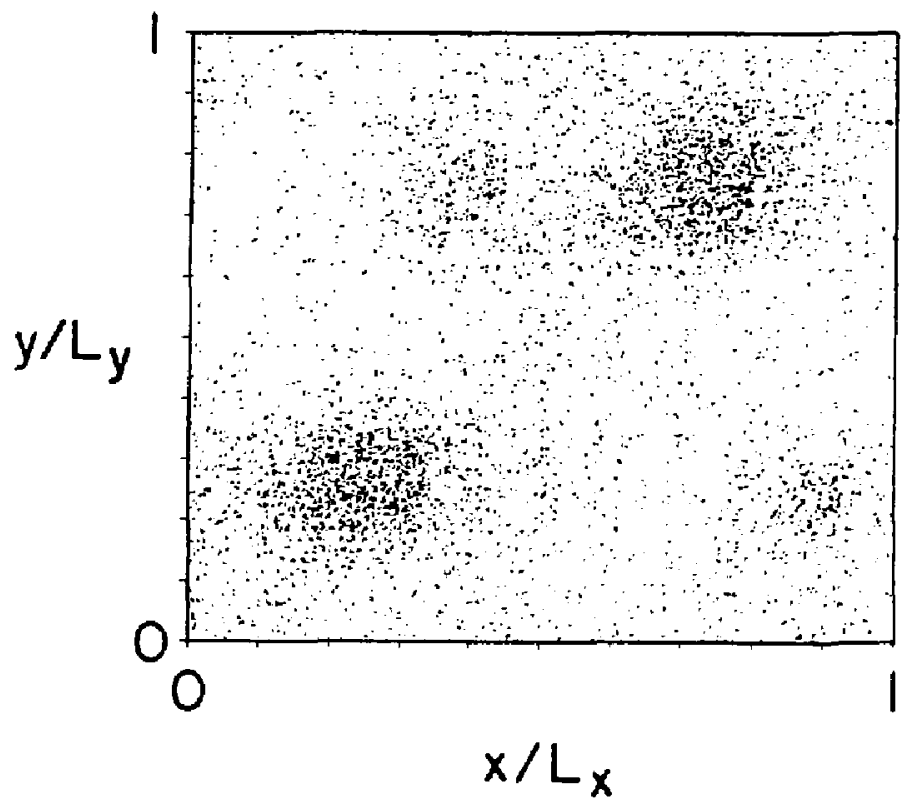

Fig. 15 


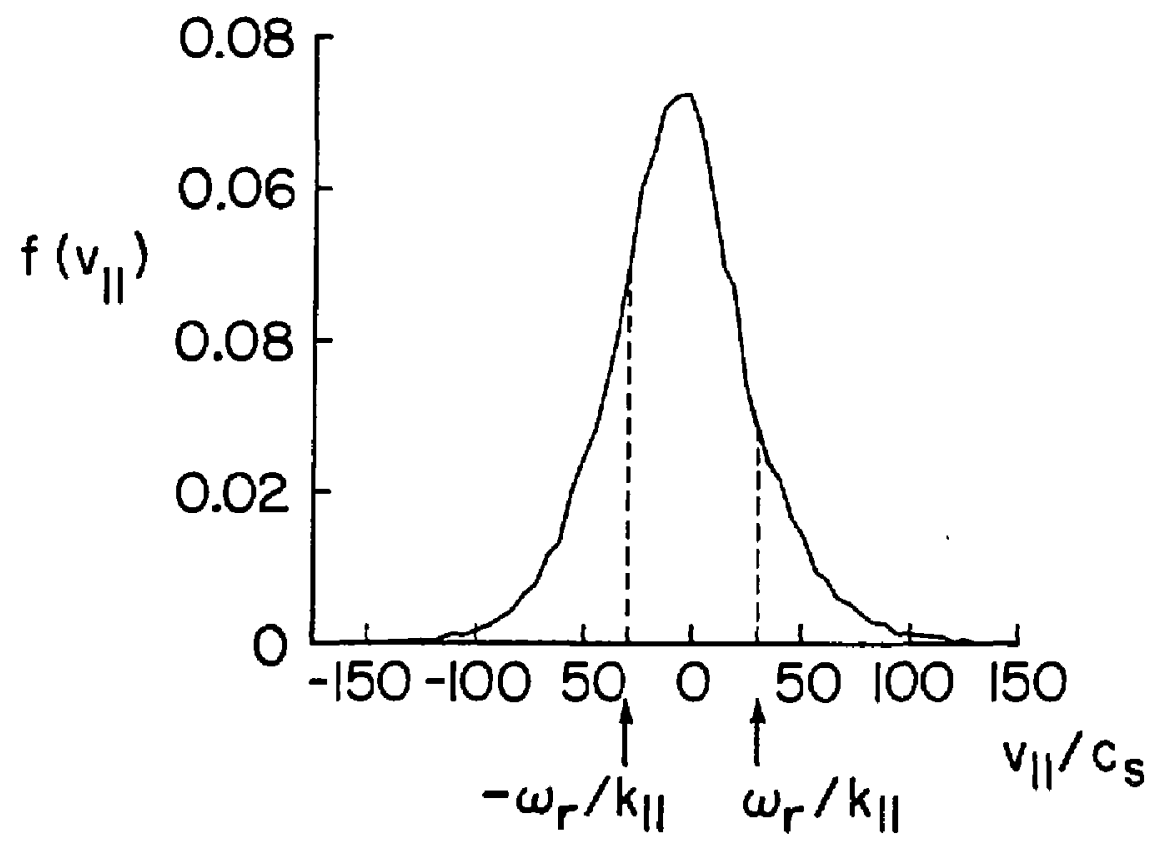

Fig. 16 
(a)

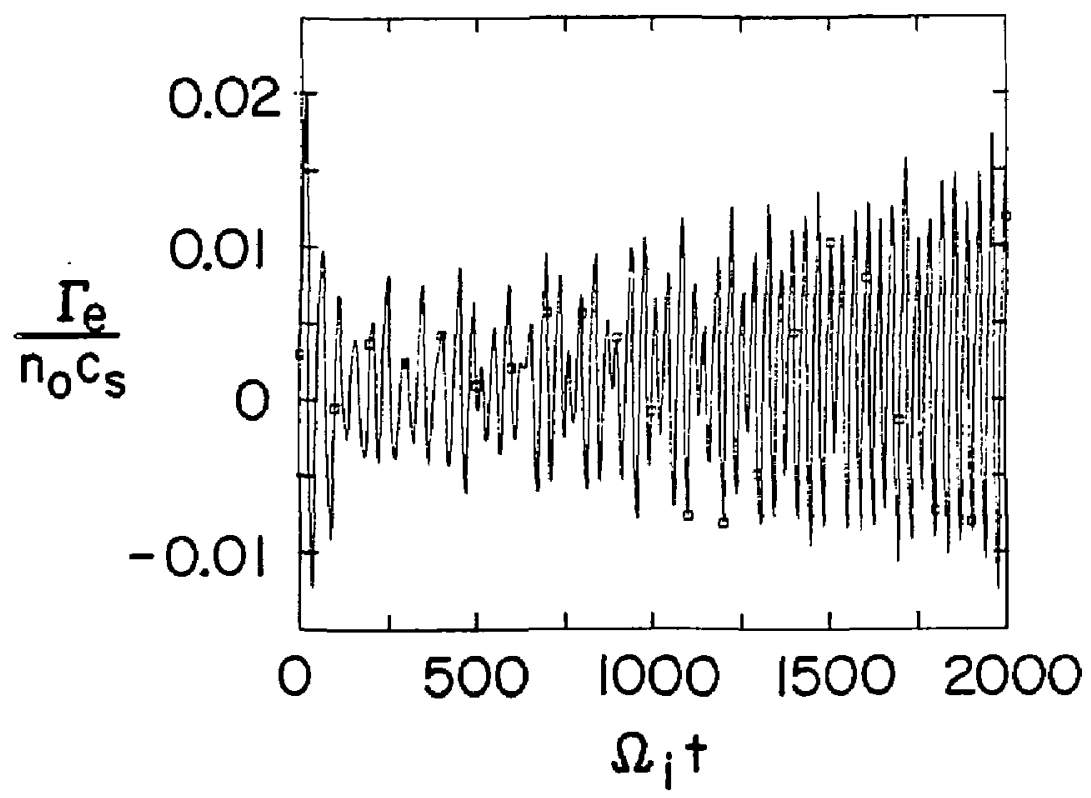

(b)

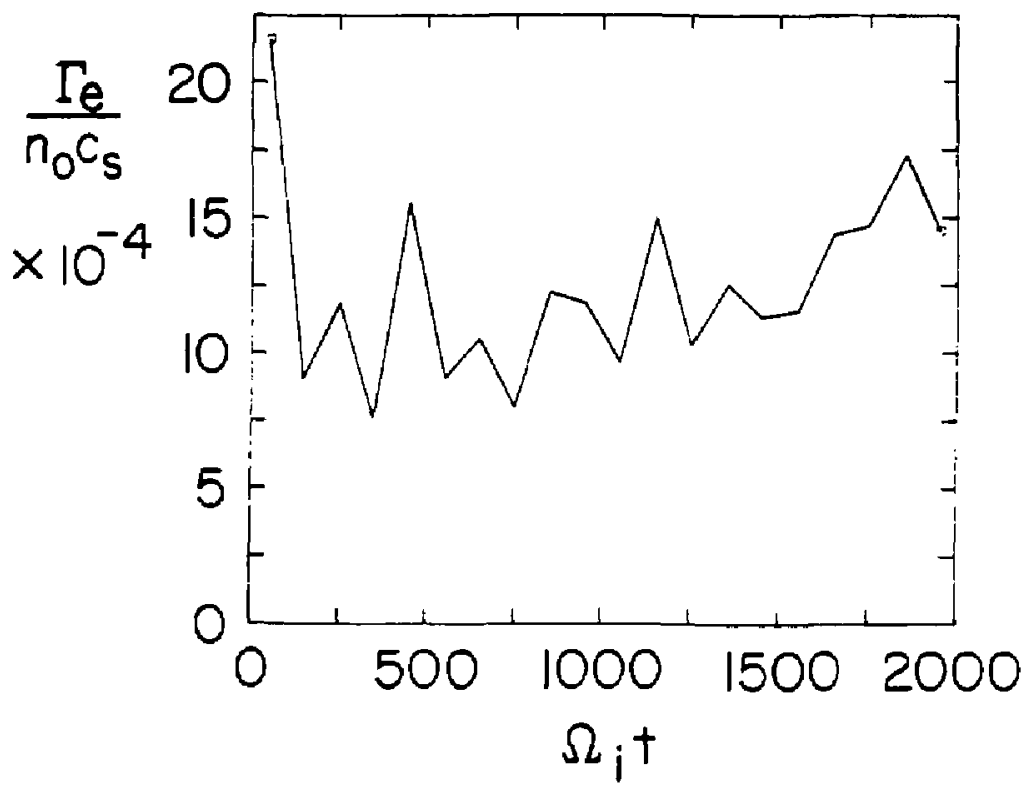

Fig. 17 


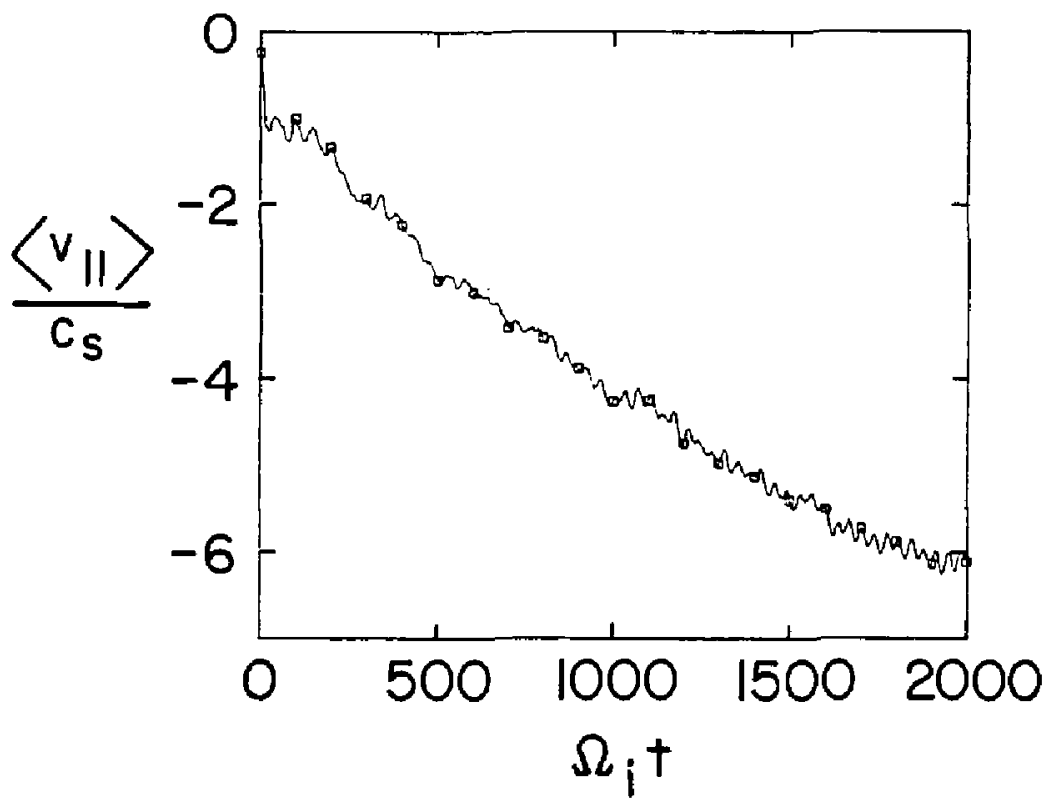

Fig. 18 


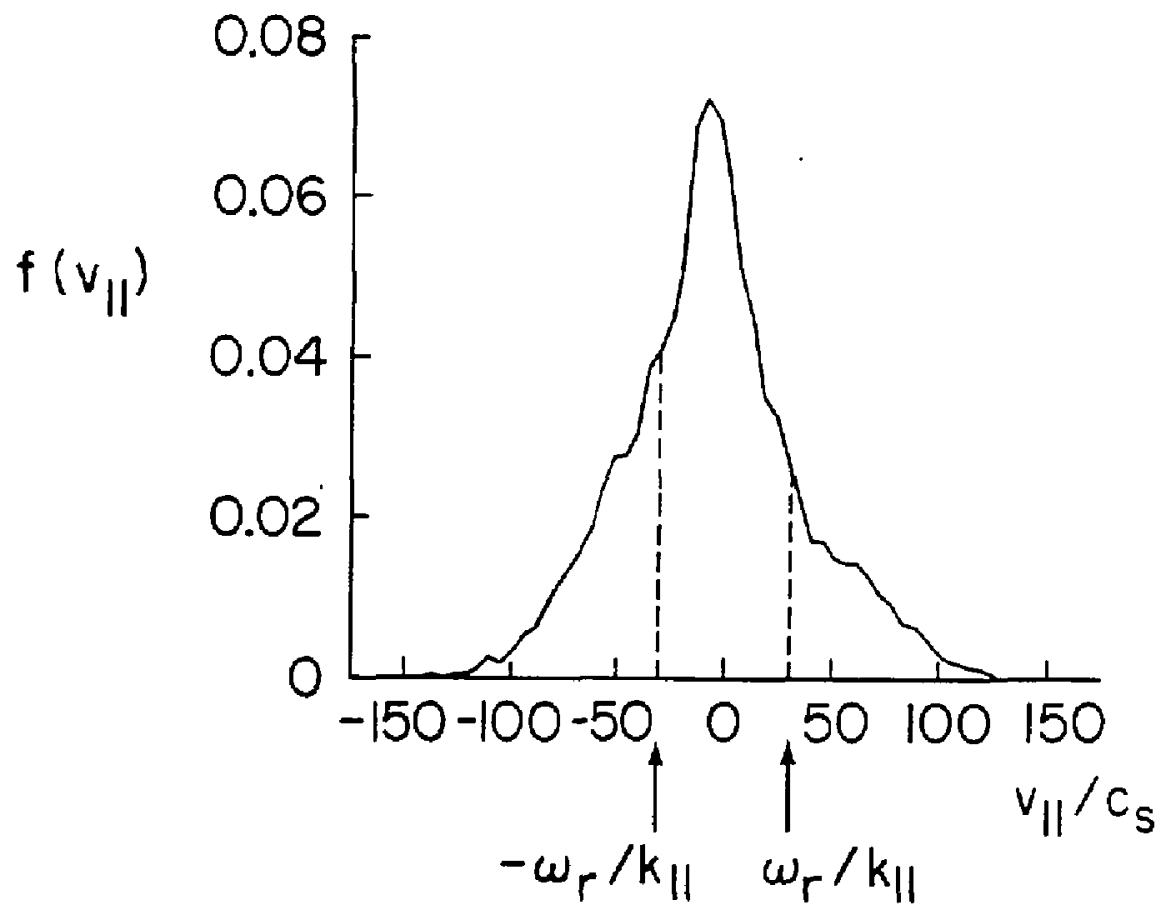

Fig. 19 


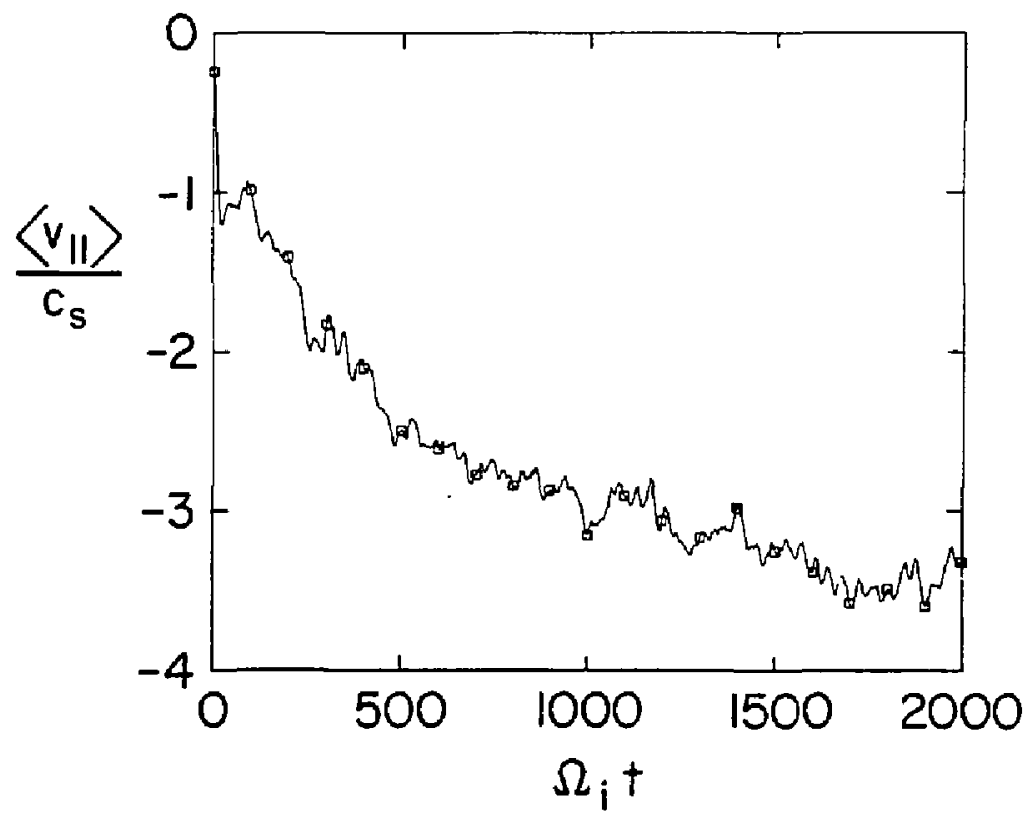

Fig. 20 
(a)
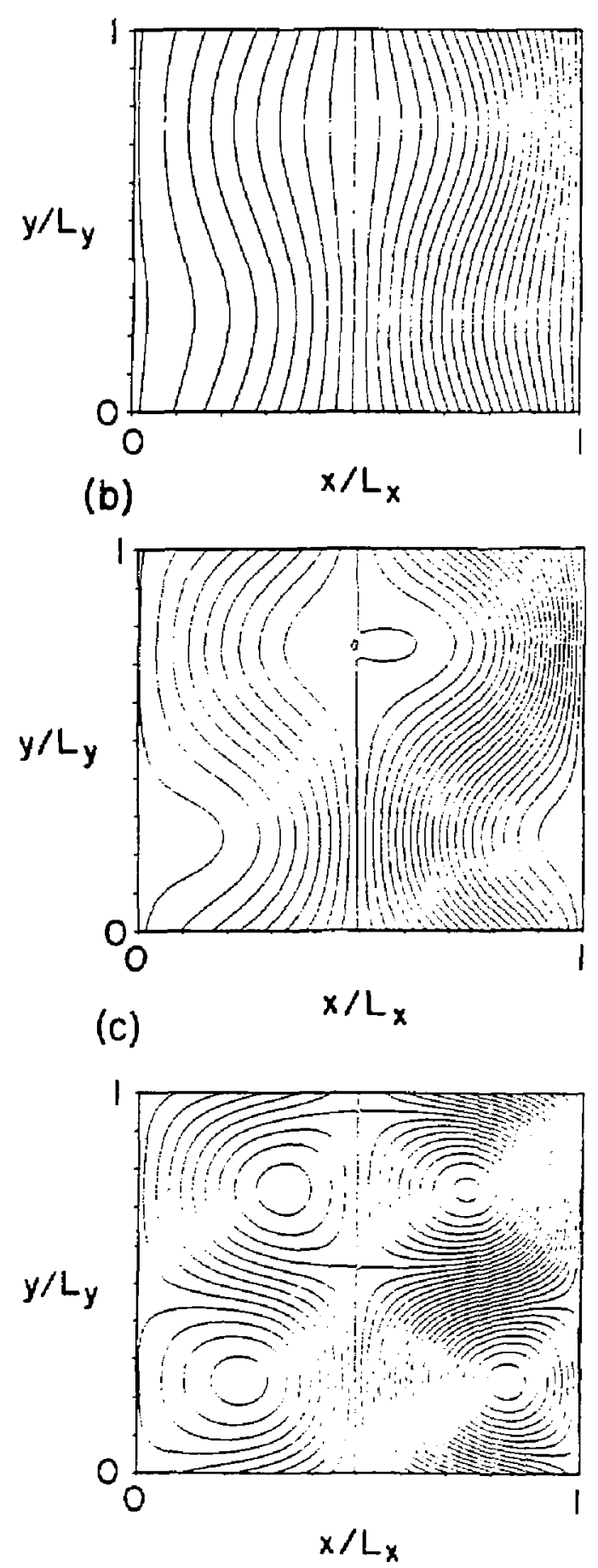

Fig. 21 


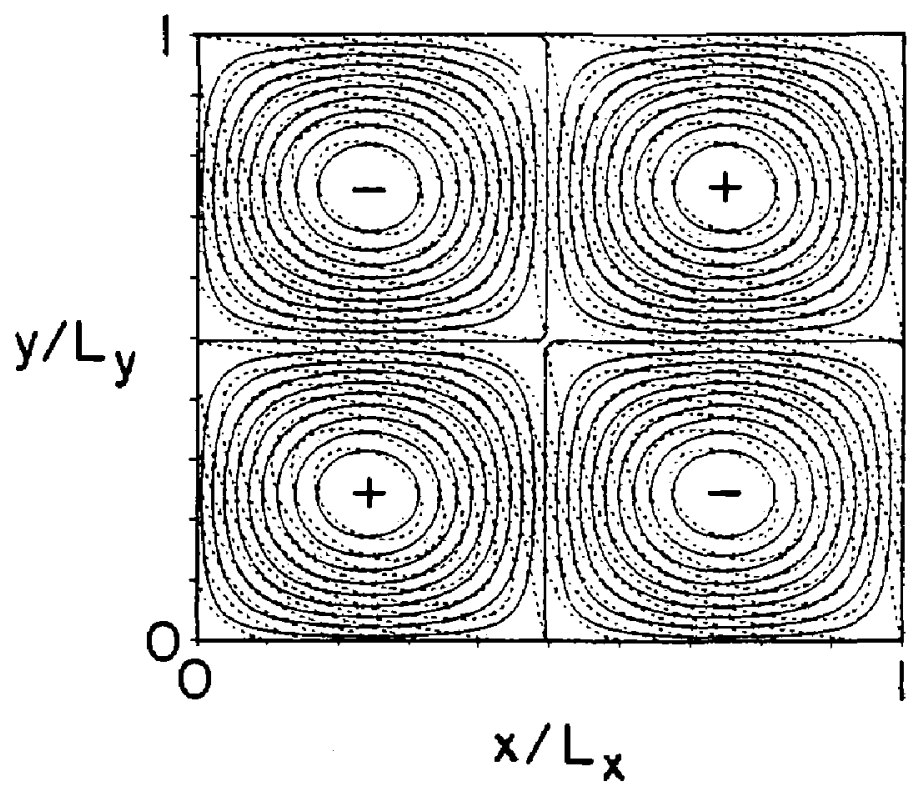

Fig. 22 


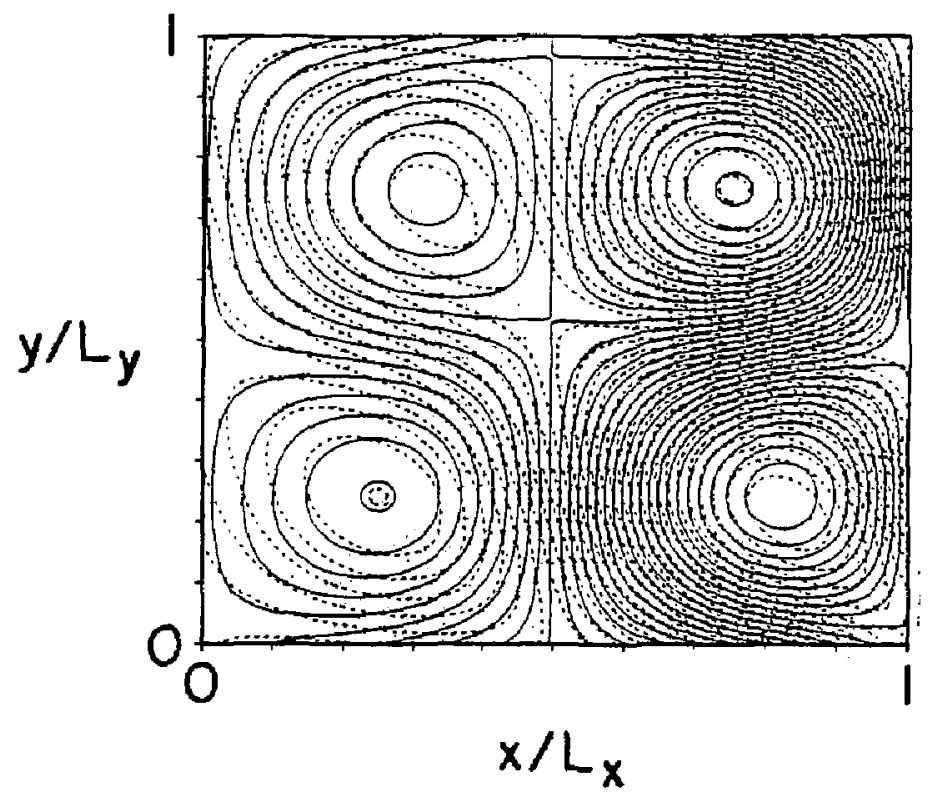

Fig. 23 


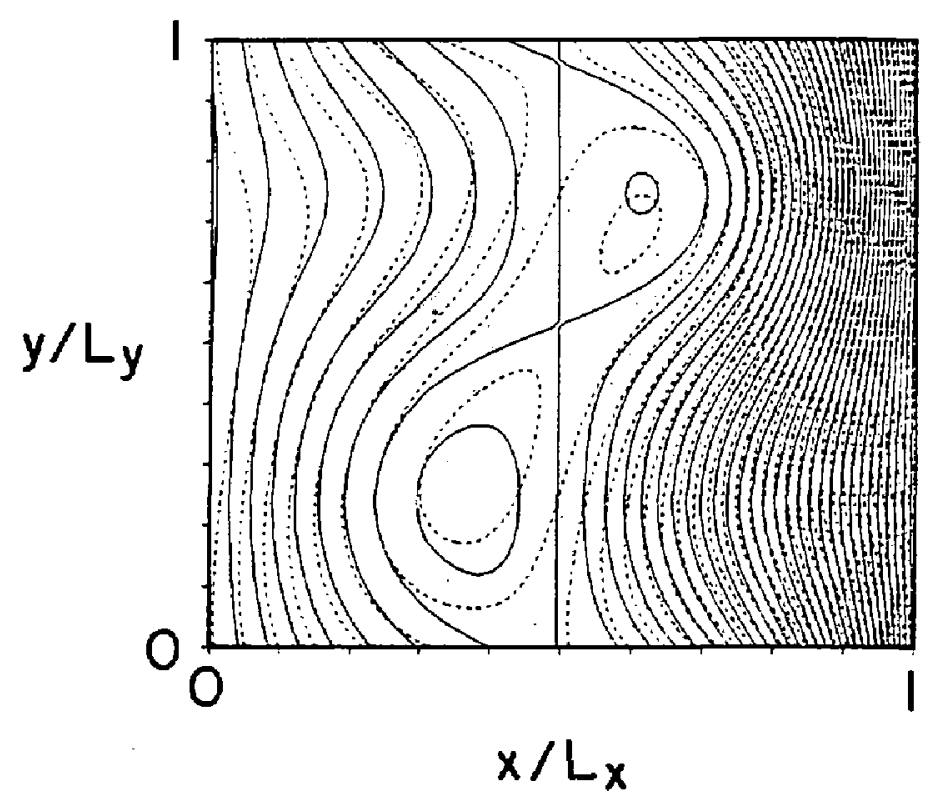

Fig. 24 


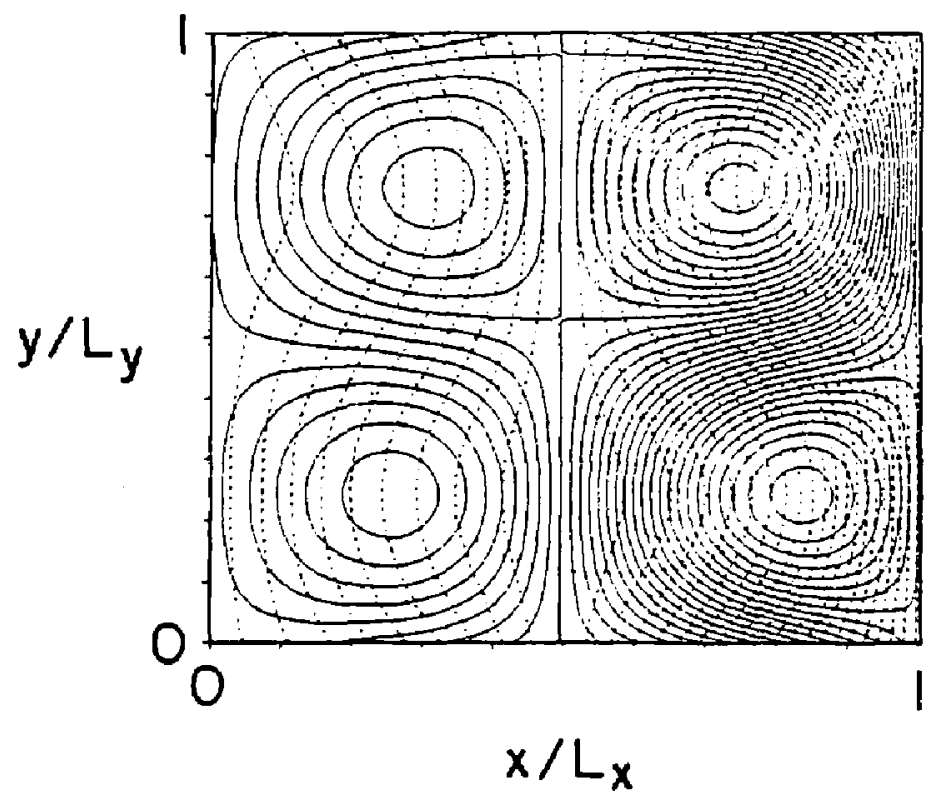

Fig. 25 


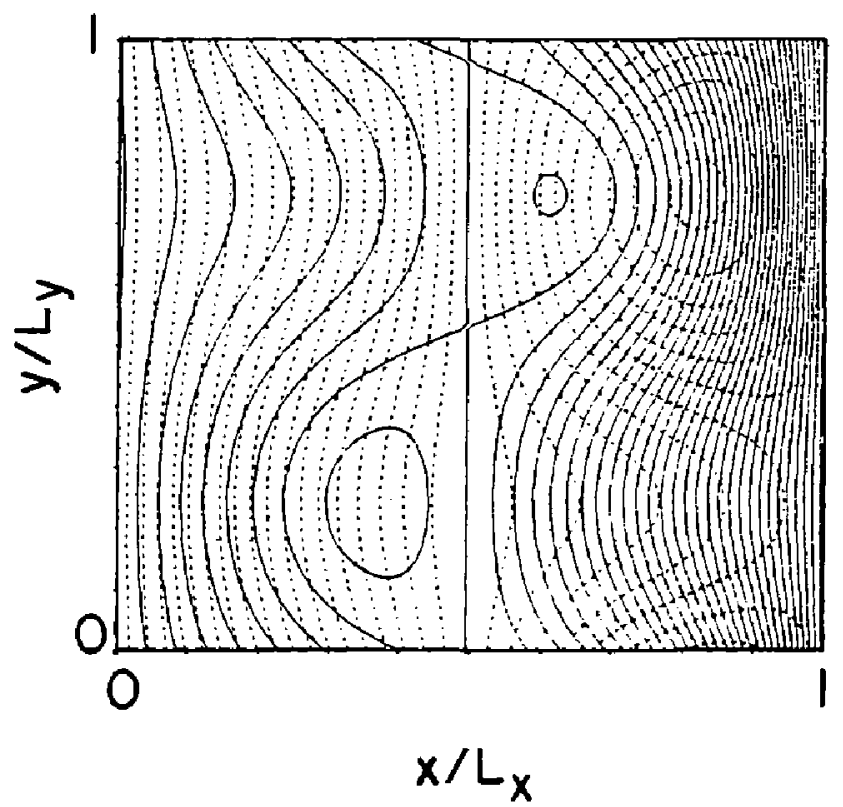

Fig. 26 


\section{\#88X0065}

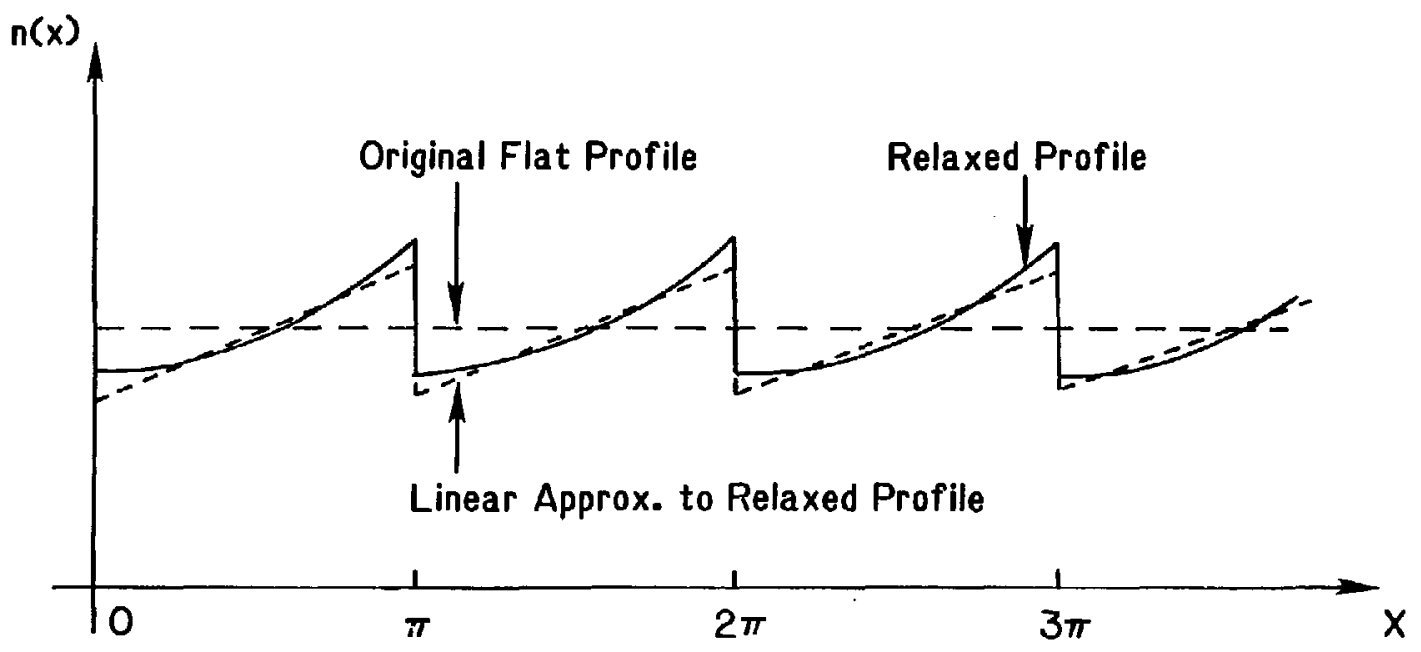

Fig. 27 


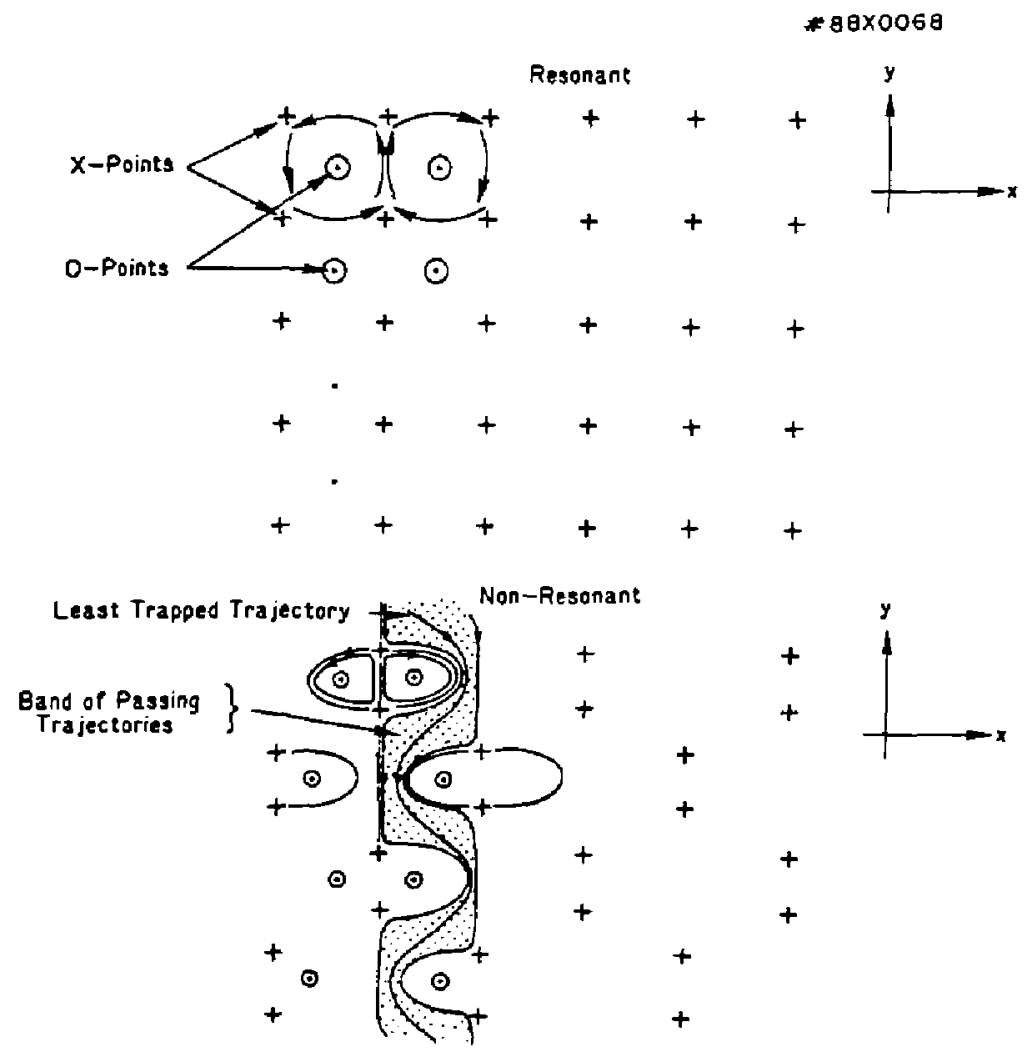

Fig. 28 
\# 88X0062

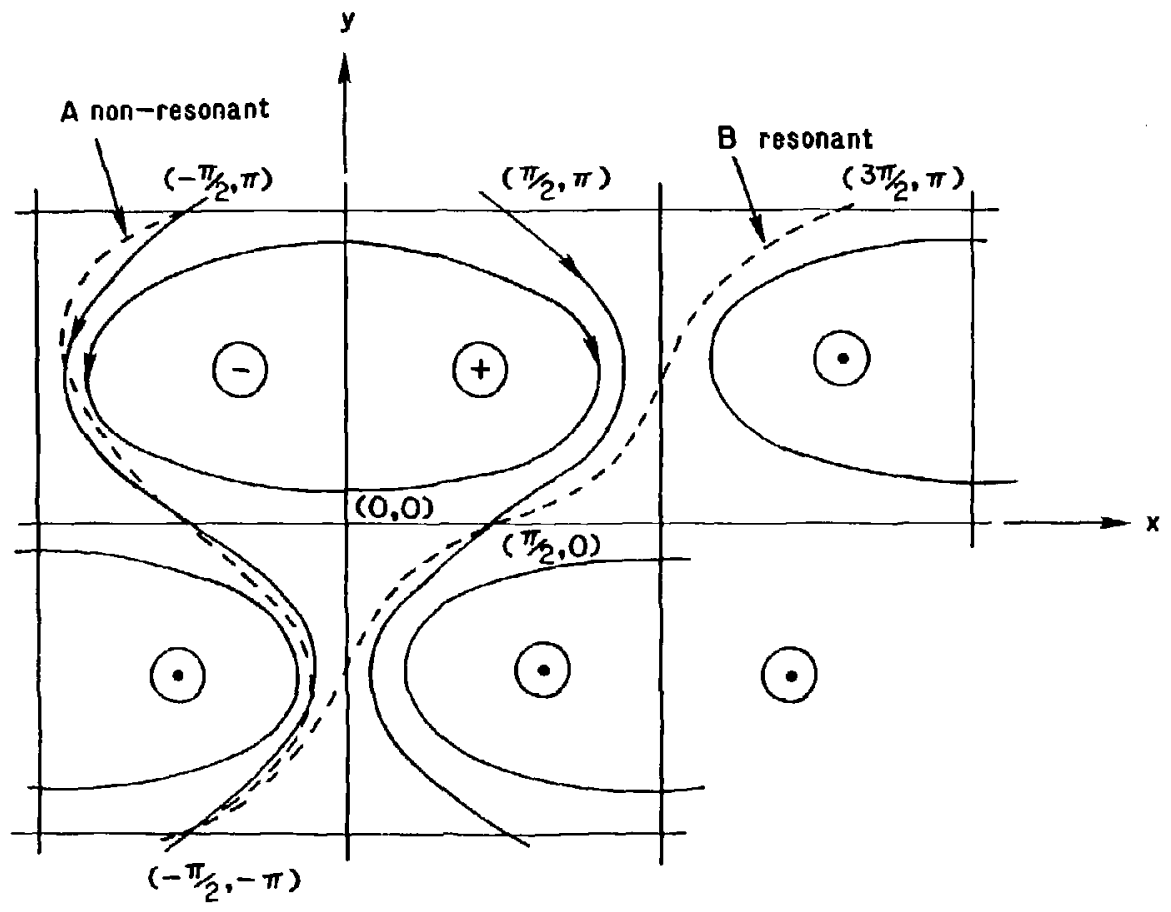

Fig. 20 
\#88x0069

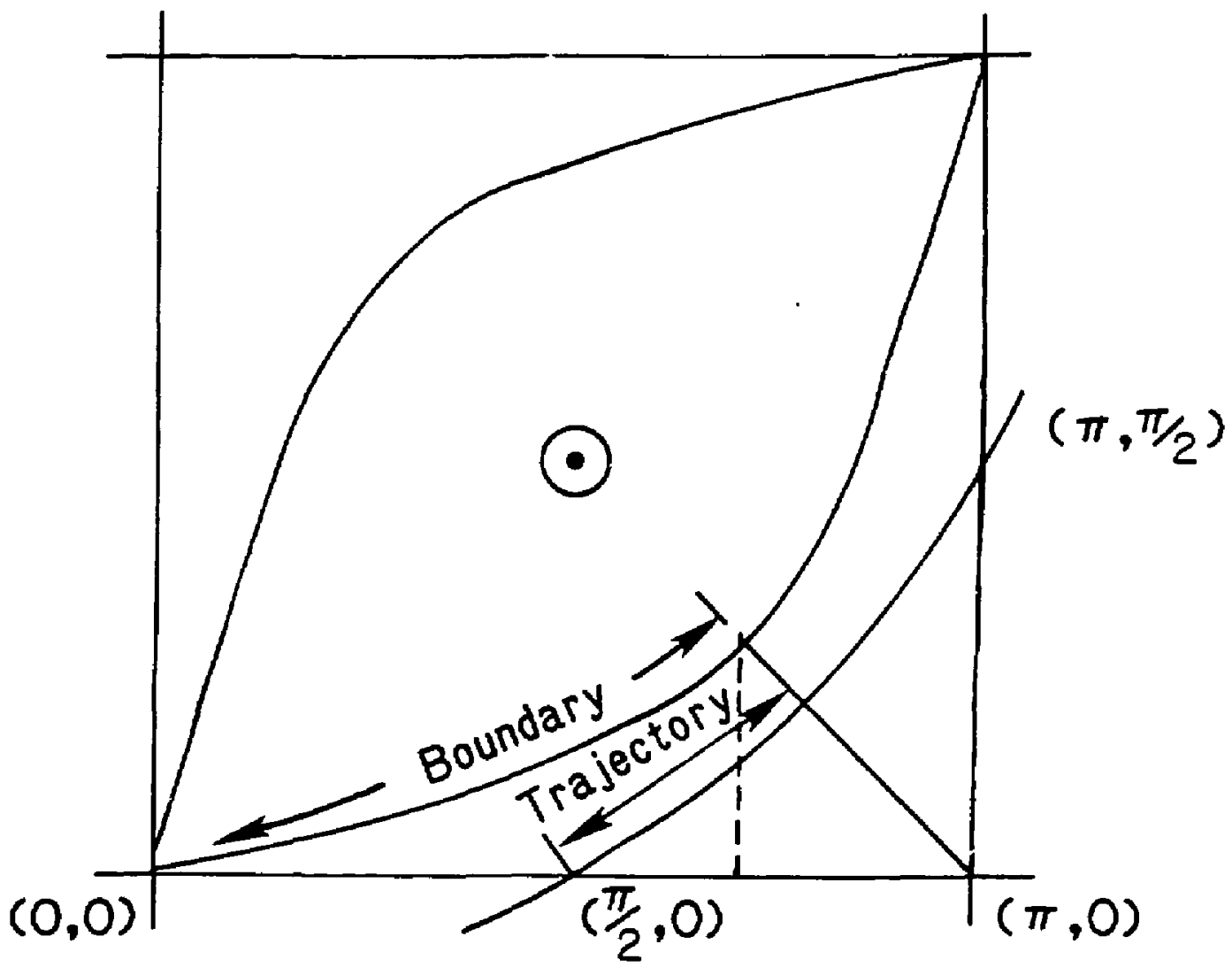

Fig. 30 


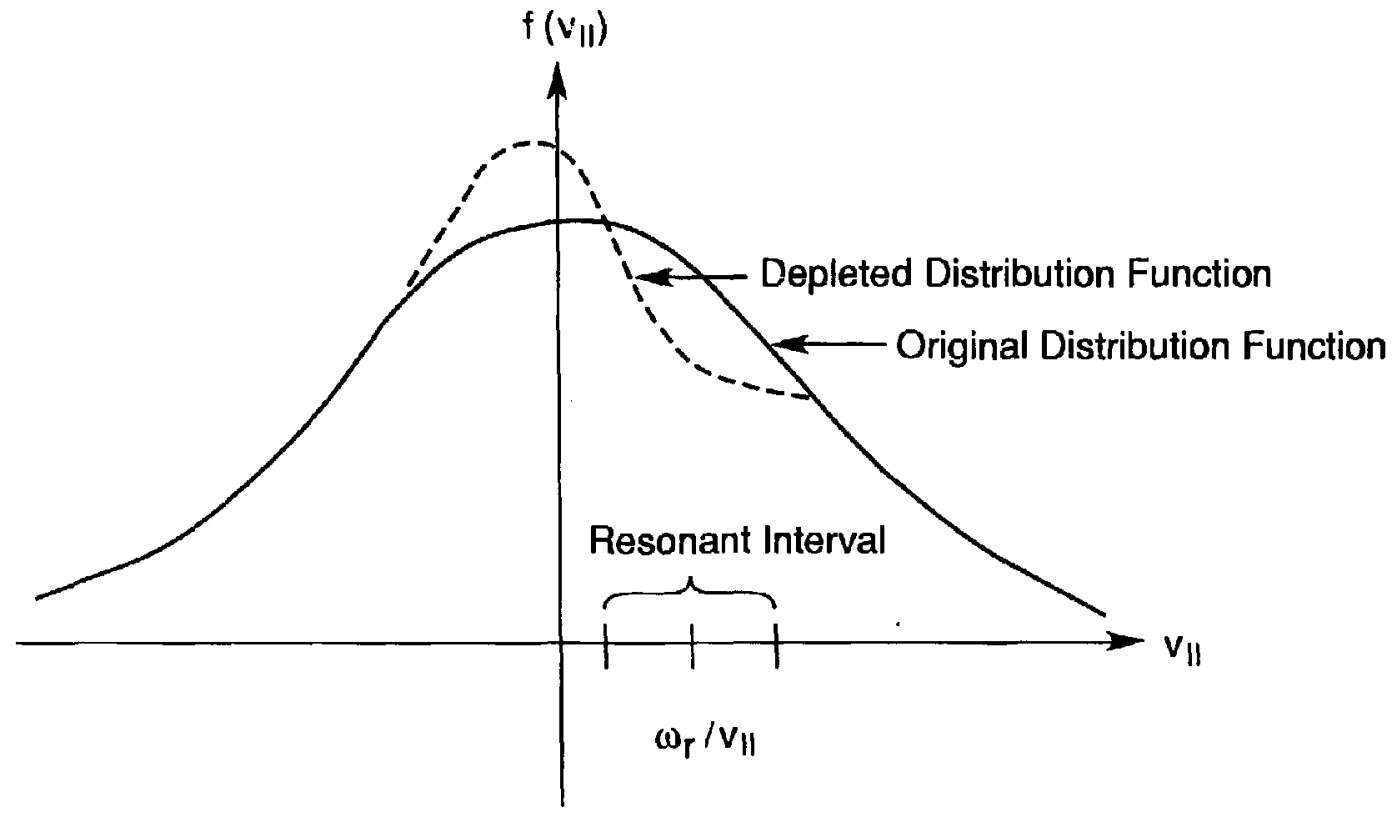




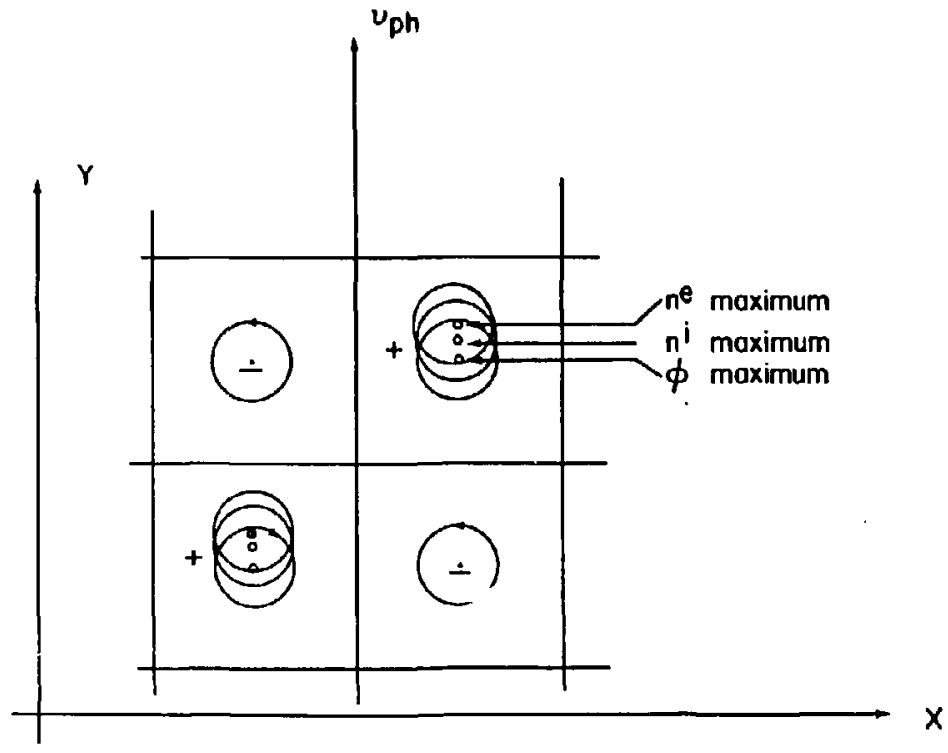

Fir. 32 